\title{
Color associations to emotion and emotion-laden words: A collection of norms for stimulus construction and selection
}

\author{
Tina M. Sutton ${ }^{1}$ • Jeanette Altarriba ${ }^{2}$
}

Published online: 19 May 2015

(C) Psychonomic Society, Inc. 2015

\begin{abstract}
Color has the ability to influence a variety of human behaviors, such as object recognition, the identification of facial expressions, and the ability to categorize stimuli as positive or negative. Researchers have started to examine the relationship between emotional words and colors, and the findings have revealed that brightness is often associated with positive emotional words and darkness with negative emotional words (e.g., Meier, Robinson, \& Clore, Psychological Science, 15, 82-87, 2004). In addition, words such as anger and failure seem to be inherently associated with the color red (e.g., Kuhbandner \& Pekrun). The purpose of the present study was to construct norms for positive and negative emotion and emotion-laden words and their color associations. Participants were asked to provide the first color that came to mind for a set of 160 emotional items. The results revealed that the color RED was most commonly associated with negative emotion and emotion-laden words, whereas YELLOW and WHITE were associated with positive emotion and emotion-laden words, respectively. The present work provides researchers with a large database to aid in stimulus construction and selection.
\end{abstract}

Keywords Emotion words · Emotion-laden words · Color associations

Tina M. Sutton

tmsgsh@rit.edu

1 Department of Psychology, Rochester Institute of Technology, Rochester, NY, USA

2 Department of Psychology, University at Albany, State University of New York, Albany, NY, USA
Research has indicated that individuals often associate colors with various emotional terms. For example, when participants are asked to associate an emotional response with a color, they often associate bright colors (e.g., white, pink) with positive emotions (e.g., happy, relaxed) and dark colors (e.g., black, brown) with negative emotions (anxious, sad) (Hemphill, 1996). Meier, Robinson, and Clore (2004) found that we tend to infer the valence of a stimulus (good or bad) on the basis of brightness. In a series of studies, participants were asked to decide whether a word presented was negative or positive. Half of the words were positive in valence, and the other half were negative. In addition, the words were presented in black and white. The results revealed that participants were faster to categorize the positive words when they appeared in white than when they appeared in black, and the opposite was true for the negative words. These results support the existence of an association between brightness and valence, and this association seems to automatically influence response times. Research has consistently shown that stimulus valence is processed quickly and automatically (e.g., Baddeley, 1972; Vuilleumier, 2005). The hue of a stimulus is also processed at a very early stage of perception (Gegenfurtner \& Kiper, 2003). When the color of the stimulus (e.g., white) matches the valence of the stimulus (e.g., positive), individuals are able to perceive and categorize the stimulus more efficiently.

Work by Gohar (2008) has demonstrated that color is an important perceptual feature of six basic emotions: anger, disgust, fear, happiness, sadness, and surprise. Participants were asked to provide up to eight perceptual features for each of the six basic emotions. For example, participants were asked, "If 'anger' was an object or something tangible, palpable that you can hold in your hands, how would you describe it or what would it look like?" (Gohar, 2008, p. 79). The results revealed that color was often listed as a perceptual feature, and it was usually listed as the first or second feature in the list. The color 
red was most associated with anger, green with disgust, black with fear, yellow with happiness, blue with sadness, and bright with surprise. These associations may be a result of various expressions containing color terms that are used in the English language - for example, "seeing red" or "feeling blue."

Colors are often used to signify information in our environment. For example, the color red generally conveys information regarding threat (e.g., alarms; Travis, 1991) and failure, particularly in achievement settings (e.g., the red ink used by teachers to correct mistakes; Moller, Elliot, \& Maier, 2009). Recently, researchers have investigated the relationship between the color red and its ability to influence behavior. Moller et al. (2009) found that the color red is positively associated with negative words, as well as with words specific to failure. Young, Elliot, Feltman, and Ambady (2013) examined the ability of the color red to influence the identification of negative facial expressions, specifically anger and fear. Participants were asked to categorize faces displaying happiness and anger (Exp. 1) or fear and anger (Exp. 2) after viewing a fixation cross that was replaced by a red, green (Exp. 1), blue (Exp. 2), or gray background. Their results revealed that red enhanced the identification of angry faces in both Experiments 1 and 2. Red did not enhance the identification of fear, indicating that red is specific to threat and not associated with negative emotion in general.

Color has also been shown to influence the emotional memory enhancement effect: better recall and recognition of emotional than of neutral stimuli. Kuhbandner and Pekrun (2013) presented participants with 30 lists of words. Twentyseven of those lists contained a series of 12 words, 11 appearing in black ink and one appearing in red, green, or blue ink. The remaining three lists contained 12 words, all presented in black ink. Nine of the colored words were negative in valence, nine were positive in valence, and nine were neutral. Participants were asked to study the lists and then to recall all of the words they remembered. The results revealed the standard emotional memory enhancement effect - the emotional words were recalled more often than the neutral words - and the typical von Restorff effect-memory for the colored words was better than memory for the words presented in black ink. The finding of particular interest was the Color $\times$ Emotional Valence interaction: Memory for neutral words was not influenced by color, but the color red enhanced memory for the negative words, and the color green enhanced memory for the positive words.

Sutton and Altarriba (2008) examined a subset of emotion words associated with specific colors, indicated in the University of South Florida (USF) word norm database, using the emotional Stroop task. In a series of two studies, participants completed a Stroop task, viewing color words (e.g., green, blue), color-related neutral words (e.g., frog, sky), and color-related emotion words (e.g., envy, sad). Each word type was presented in a congruent color and an incongruent color, in order to examine the sizes of the Stroop effect for each of the three word types. The results revealed that the color words produced the largest Stroop interference effect, but of more interest, the color-related neutral words and color-related emotion words produced same-size Stroop interference effects. These results suggest that emotion words automatically activate color associations, and these color associations can interfere with the current task. These results are consistent with a network notion of memory (Collins \& Loftus 1975), suggesting that color nodes may be linked with emotion nodes in memory.

It appears that we often associate colors with emotions, and that these associations can influence a variety of behaviors, such as response time, memory, and facial identification. The goal of the present work was to establish a set of color norms for a variety of emotional stimuli. These stimuli included both emotion words and emotion-laden words. An emotion word labels an emotional state (e.g., happy, sad), whereas emotionladen words have an emotional connotation (make you feel a certain way), but do not directly label an emotional state. Research has indicated that these two types of emotional stimuli are processed differently, with emotion words often producing stronger effects in tasks such as the affective Simon task (Altarriba \& Basnight-Brown, 2011; Knickerbocker \& Altarriba, 2013).

The purpose of this study was to provide researchers with a set of color norms for emotional stimuli to be used in perception, language, memory, and cognition studies in paradigms such as the Stroop task, the rapid visual serial presentation (RSVP) paradigm, the attentional dot probe task, the affective Simon task, and so forth. Understanding the relationship between an emotional stimulus and other perceptual features, such as color, is important when designing studies, or investigating the representation of emotion in memory. The present work may also speak to the need to consider what color stimuli researchers use in their experimental paradigms to distinguish one stimulus from another, or one response from another: The color may actually act as a confounding variable if there is an association between the color and the stimulus or response.

\section{Method}

\section{Participants}

One hundred and seven University at Albany, State University of New York, undergraduates completed the present study for extra credit or research participation credit in their psychology course. Ninety-six of the participants were native English speakers, and all of the participants reported using English as their primary language. Most participants reported using English $100 \%$ of the day. Two of the participants reported 
being color blind, and their data were not included in the present study. Of the remaining participants, 75 were female.

\section{Materials}

Thirty-five negative emotion words, 55 negative emotionladen words, 29 positive emotion words, and 41 positive emotion-laden words were selected from the Affective Norms for English Words (ANEW) database. ANEW is a set of English words rated for pleasure (valence), arousal, and dominance on a scale from 1 (negative, low arousal, low dominance) to 9 (positive, high arousal, high dominance). The negative words selected for the present study received pleasure ratings below 4 , and the positive words received pleasure ratings above 7 . The average pleasure rating for the negative emotion words was $2.52(S D=0.58)$, that for the negative emotion-laden words was $2.24(S D=0.51)$, that for the positive emotion words was $7.71(S D=0.60)$, and that for the positive emotion-laden words was $7.82(S D=0.45)$. The emotional stimuli were embedded among 234 emotionally neutral words. All of the emotional words were moderate to high in arousal, except for three of the positive emotion words (i.e., relaxed, secure, and safe) and one of the positive emotion-laden words (i.e., peace) (see Table 1).

All participants also completed a Language History Questionnaire (LHQ). The LHQ asked participants to report where they were born, how long they had been living in the United States, the number of languages they spoke, and the percentage of the day that they spent speaking each language. Participants were also asked to rate their spoken, written, and conversational proficiency in English on a scale from 1 to 10.

\section{Procedure}

Upon arrival, participants were asked to read, sign, and date the informed-consent form prior to receiving the packet containing the emotional and neutral words as well as the LHQ. Participants completed the color task first. The 390 words were randomly mixed together, and participants were asked to write down the first color that came to mind for each word in the packet. Participants were provided with the following set of directions:

For each word provided, please write down the first color that comes to mind. For example, if you see the word CLOUD, you might write "blue," "white," or "gray." If you cannot think of a color, or you don't believe a color is associated with the word provided, you may leave the space blank. If you have any questions, please ask them now.

Upon completion of the color task, participants filled out the LHQ. The entire task took $30-45 \mathrm{~min}$ to complete.
Table 1 Pleasure (valence) and arousal ratings from the Affective Norms for English Words for the emotional stimuli, on a scale from 1 (pleasant, low arousal) to 9 (unpleasant, high arousal)

\begin{tabular}{|c|c|c|c|}
\hline Word Type & Word & Pleasure & Arousal \\
\hline \multirow[t]{35}{*}{ Negative emotion } & Afraid & 2 & 6.67 \\
\hline & Agony & 2.43 & 6.06 \\
\hline & Angry & 2.85 & 7.17 \\
\hline & Anguished & 2.12 & 5.33 \\
\hline & Contempt & 3.85 & 5.28 \\
\hline & Cruel & 1.97 & 5.68 \\
\hline & Depressed & 1.83 & 4.72 \\
\hline & Discouraged & 3 & 4.53 \\
\hline & Disgusted & 2.45 & 5.42 \\
\hline & Dreadful & 2.26 & 5.84 \\
\hline & Embarrassed & 3.03 & 5.87 \\
\hline & Enraged & 2.46 & 7.97 \\
\hline & Fear & 2.76 & 6.96 \\
\hline & Frustrated & 2.48 & 5.61 \\
\hline & Guilty & 2.63 & 6.04 \\
\hline & Helpless & 2.2 & 5.34 \\
\hline & Hostile & 2.73 & 6.44 \\
\hline & Jealousy & 2.52 & 6.36 \\
\hline & Lonely & 2.17 & 4.51 \\
\hline & Mad & 2.44 & 6.76 \\
\hline & Misery & 1.93 & 5.17 \\
\hline & Moody & 3.2 & 4.18 \\
\hline & Nervous & 3.29 & 6.59 \\
\hline & Panic & 3.12 & 7.02 \\
\hline & Rage & 2.41 & 8.17 \\
\hline & Regretful & 2.28 & 5.74 \\
\hline & $\mathrm{Sad}$ & 1.61 & 4.13 \\
\hline & Scared & 2.78 & 6.82 \\
\hline & Shamed & 2.5 & 4.88 \\
\hline & Suspicious & 3.76 & 6.29 \\
\hline & Tense & 3.56 & 6.53 \\
\hline & Terrified & 1.72 & 7.86 \\
\hline & Troubled & 2.17 & 5.94 \\
\hline & Unhappy & 1.57 & 4.18 \\
\hline & Upset & 2 & 5.86 \\
\hline \multirow[t]{12}{*}{ Negative emotion-laden } & Abduction & 2.76 & 5.53 \\
\hline & Abortion & 3.5 & 5.39 \\
\hline & Abuse & 1.8 & 6.83 \\
\hline & Bees & 3.2 & 6.51 \\
\hline & Bloody & 2.9 & 6.41 \\
\hline & Burial & 2.05 & 5.08 \\
\hline & Cancer & 1.5 & 6.42 \\
\hline & Cemetery & 2.63 & 4.82 \\
\hline & Coffin & 2.56 & 5.03 \\
\hline & Corpse & 2.18 & 4.74 \\
\hline & Crash & 2.31 & 6.95 \\
\hline & Criminal & 2.93 & 4.79 \\
\hline
\end{tabular}


Table 1 (continued)

\begin{tabular}{|c|c|c|c|}
\hline Word Type & Word & Pleasure & Arousal \\
\hline & Crisis & 2.74 & 5.44 \\
\hline & Danger & 2.95 & 7.32 \\
\hline & Dead & 1.94 & 5.73 \\
\hline & Death & 1.61 & 4.59 \\
\hline & Demon & 2.11 & 6.76 \\
\hline & Disaster & 1.73 & 6.33 \\
\hline & Divorce & 2.22 & 6.33 \\
\hline & Funeral & 1.39 & 4.94 \\
\hline & Gun & 3.47 & 7.02 \\
\hline & Hell & 2.24 & 5.38 \\
\hline & Illness & 2.48 & 4.71 \\
\hline & Infection & 1.66 & 5.03 \\
\hline & Injury & 2.49 & 5.69 \\
\hline & Insult & 2.29 & 6 \\
\hline & Jail & 1.95 & 5.49 \\
\hline & Killer & 1.89 & 7.86 \\
\hline & $\mathrm{Lie}$ & 2.79 & 5.96 \\
\hline & Massacre & 2.28 & 5.33 \\
\hline & Morbid & 2.87 & 5.06 \\
\hline & Nightmare & 1.91 & 7.59 \\
\hline & Pain & 2.13 & 6.5 \\
\hline & Poison & 1.98 & 6.05 \\
\hline & Pollute & 1.85 & 6.08 \\
\hline & Poverty & 1.67 & 4.87 \\
\hline & Punishment & 2.22 & 5.93 \\
\hline & Rape & 1.25 & 6.81 \\
\hline & Robber & 2.61 & 5.62 \\
\hline & Sick & 1.9 & 4.29 \\
\hline & Sickness & 2.25 & 5.61 \\
\hline & Slave & 1.84 & 6.21 \\
\hline & Suicide & 1.25 & 6.73 \\
\hline & Tomb & 2.94 & 4.73 \\
\hline & Toothache & 1.98 & 5.55 \\
\hline & Tornado & 2.55 & 6.83 \\
\hline & Tragedy & 1.78 & 6.24 \\
\hline & Trash & 2.67 & 4.16 \\
\hline & Trauma & 2.1 & 6.33 \\
\hline & Ugly & 2.43 & 5.38 \\
\hline & Ulcer & 1.78 & 6.12 \\
\hline & Unfaithful & 2.05 & 6.2 \\
\hline & Victim & 2.18 & 6.06 \\
\hline & Violent & 2.29 & 6.89 \\
\hline & War & 2.08 & 7.49 \\
\hline \multirow[t]{6}{*}{ Positive emotion } & Admired & 7.74 & 6.11 \\
\hline & Affection & 8.39 & 6.21 \\
\hline & Aroused & 7.97 & 6.63 \\
\hline & Astonished & 6.56 & 6.58 \\
\hline & Beautiful & 7.6 & 6.17 \\
\hline & Brave & 7.15 & 6.15 \\
\hline
\end{tabular}

Table 1 (continued)

\begin{tabular}{|c|c|c|c|}
\hline Word Type & Word & Pleasure & Arousal \\
\hline & Cheer & 8.1 & 6.12 \\
\hline & Confident & 7.98 & 6.22 \\
\hline & Elated & 7.45 & 6.21 \\
\hline & Friendly & 8.43 & 5.11 \\
\hline & Grateful & 7.37 & 4.58 \\
\hline & Happy & 8.21 & 6.49 \\
\hline & Hopeful & 7.1 & 5.78 \\
\hline & Inspired & 7.15 & 6.02 \\
\hline & Joy & 8.6 & 7.22 \\
\hline & Joyful & 8.22 & 5.98 \\
\hline & Kind & 7.59 & 4.46 \\
\hline & Love & 8.72 & 6.44 \\
\hline & Lust & 7.12 & 6.88 \\
\hline & Merry & 7.9 & 5.9 \\
\hline & Nice & 6.55 & 4.38 \\
\hline & Passion & 8.03 & 7.26 \\
\hline & Proud & 8.03 & 5.56 \\
\hline & Relaxed & 7 & 2.39 \\
\hline & Safe & 7.07 & 3.86 \\
\hline & Satisfied & 7.94 & 4.94 \\
\hline & Secure & 7.57 & 3.14 \\
\hline & Surprised & 7.47 & 7.47 \\
\hline & Triumphant & 8.82 & 6.78 \\
\hline \multirow[t]{26}{*}{ Positive emotion-laden } & Achievement & 7.89 & 5.53 \\
\hline & Adorable & 7.81 & 5.12 \\
\hline & Baby & 8.22 & 5.53 \\
\hline & Birthday & 7.84 & 6.68 \\
\hline & Bouquet & 7.02 & 5.46 \\
\hline & Cash & 8.37 & 7.37 \\
\hline & Champion & 8.44 & 5.85 \\
\hline & Church & 6.28 & 4.34 \\
\hline & Diamond & 7.92 & 5.53 \\
\hline & Diploma & 8 & 5.67 \\
\hline & Engaged & 8 & 6.77 \\
\hline & Freedom & 7.58 & 5.52 \\
\hline & Friend & 7.74 & 5.74 \\
\hline & Gift & 7.77 & 6.14 \\
\hline & God & 8.15 & 5.95 \\
\hline & Grin & 7.4 & 5.27 \\
\hline & Heaven & 7.3 & 5.61 \\
\hline & Holiday & 7.55 & 6.59 \\
\hline & Jewel & 7 & 5.38 \\
\hline & Joke & 8.1 & 6.74 \\
\hline & Kiss & 8.26 & 7.32 \\
\hline & Laughter & 8.45 & 6.75 \\
\hline & Lucky & 8.17 & 6.53 \\
\hline & Millionaire & 8.03 & 6.14 \\
\hline & Miracle & 8.6 & 7.65 \\
\hline & Money & 7.59 & 5.7 \\
\hline
\end{tabular}


Table 1 (continued)

\begin{tabular}{llll}
\hline Word Type & Word & Pleasure & Arousal \\
\hline Outdoors & 7.47 & 5.92 \\
Peace & 7.72 & 2.95 \\
Pretty & 7.75 & 6.03 \\
Rescue & 7.7 & 6.53 \\
Riches & 7.7 & 6.17 \\
Romantic & 8.32 & 7.59 \\
Thrill & 8.05 & 8.02 \\
Treasure & 8.27 & 6.75 \\
Triumph & 7.8 & 5.78 \\
Trophy & 7.78 & 5.39 \\
Vacation & 8.16 & 5.64 \\
Victory & 8.32 & 6.63 \\
Waterfall & 7.88 & 5.37 \\
Wedding & 7.82 & 5.97 \\
Wish & 7.09 & 5.16 \\
\hline
\end{tabular}

Participants completed these tasks in a group setting, with 1020 participants in each session.

\section{Results}

Not all participants provided a response for every word presented. Looking at the data, clear patterns emerged for the various emotional stimuli. The most common color response for the negative emotion words was RED $(57.10 \%)$, followed by BLUE (20\%), BLACK (14.3\%), GREEN (5.70\%), and WHITE $(2.90 \%)$. The most common color response for the negative emotion-laden words was also RED (42.60\%), followed closely by BLACK (38.9 \%), then GRAY (5.6\%), GREEN (5.6\%), BROWN (3.7\%), YELLOW (1.9 \%), AND WHITE (1.9\%). All negative words seemed to elicit the color RED as the first response given; however, not every negative word resulted in RED as the first response given (e.g., afraid, depressed). The most common color response for positive emotion words was YELLOW (40.0\%), followed by RED (33.3\%), BLUE (20\%), PINK (3.3\%), and WHITE (3.3\%), and the most common response for the positive emotion-laden words was WHITE $(27.3 \%)$, followed by RED (20.5\%), GREEN (13.6 \%), BLUE (13.6\%), YELLOW (9.10\%), GOLD (9.10\%), and PINK (6.8 \%) (see Table 6).

To assess reliability, the original sample was randomly divided into two subsamples (Sample A and Sample B), and the color responses for each individual word were tallied for each subsample (see Table 7). The most common responses given for the various word types (negative emotion, negative emotion-laden, positive emotion, and positive emotion-laden) were similar for the two subsamples (see Table 7). Similar to Nelson and Schreiber (1992), we calculated the total set size (TSS) and the mean set size (MSS). TSS is the total number of different colors provided by the participants in each sample, and MSS is the total number of different color responses given by two or more participants in each sample. TSS thus includes idiosyncratic responses, but MSS does not. The TSS and MSS measures were highly correlated for all word types (see Tables 2, 3, 4, and 5 for each word type). For each of the words, the correlation between the overlapping color responses for Sample A and Sample B was correlated. The averages of these correlations were as follows: for the negative emotion words, $r=.95(S D=.05)$; for the negative emotionladen words, $r=.97(S D=.03)$; for the positive emotion words, $r=.92(S D=.07)$; and for the positive emotionladen words, $r=.93(S D=.07)$.

\section{Discussion}

The results of the present study indicate that negative and positive words are associated with different colors. All of the negative words (emotion and emotion-laden) elicited the response RED most often. This is consistent with previous research suggesting that red carries a negative meaning (Kuhbandner \& Pekrun, 2013) and is often associated with failure (Moller et al., 2009). The association between the color red and negative words and events is most likely the result of several sources. In the English language, we often use such phrases as "seeing red" (referring to feeling angry). The color red may also be associated with failure through classical conditioning, because teachers use red ink to correct mistakes (Elliot \& Maier, 2007). Elliot, Maier, Moller, Friedman, and Meinhardt (2007) found that exposure to the color red impaired participants' performance on various achievement tests, such as anagram completion and the numerical and language-based subtests of an intelligence test.

The color BLACK was also a fairly common response to the negative words in the present study. This is consistent with research examining brightness and stimulus valence (Meier et al., 2004). Darkness is often associated with negative

Table 2 Total set size (TSS) and mean set size (MSS) for the negative emotion words for Sample A and Sample B

\begin{tabular}{lllll}
\hline & $\begin{array}{l}\text { Sample } \\
\text { A MSS }\end{array}$ & $\begin{array}{l}\text { Sample } \\
\text { B MSS }\end{array}$ & $\begin{array}{l}\text { Sample } \\
\text { A TSS }\end{array}$ & $\begin{array}{l}\text { Sample } \\
\text { B TSS }\end{array}$ \\
\hline Sample A MSS & 1 & $.856^{*}$ & $.807^{*}$ & $.757^{*}$ \\
Sample B MSS & $.856^{*}$ & 1 & $.674^{*}$ & $.780^{*}$ \\
Sample A TSS & $.807^{*}$ & $.674^{*}$ & 1 & $.772^{*}$ \\
Sample B TSS & $.757^{*}$ & $.780^{*}$ & $.772^{*}$ & 1 \\
\hline
\end{tabular}

${ }^{*}$ Correlation is significant at the .01 level (two-tailed) 
Table 3 Total set size (TSS) and mean set size (MSS) for the negative emotion-laden words for Sample A and Sample B

\begin{tabular}{lllll}
\hline & $\begin{array}{l}\text { Sample } \\
\text { A MSS }\end{array}$ & $\begin{array}{l}\text { Sample } \\
\text { B MSS }\end{array}$ & $\begin{array}{l}\text { Sample } \\
\text { A TSS }\end{array}$ & $\begin{array}{l}\text { Sample } \\
\text { B TSS }\end{array}$ \\
\hline Sample A MSS & 1 & $.783^{*}$ & $.834^{*}$ & $.747^{*}$ \\
Sample B MSS & $.783^{*}$ & 1 & $.856^{*}$ & $.882^{*}$ \\
Sample A TSS & $.834^{*}$ & $.856^{*}$ & 1 & $.841^{*}$ \\
Sample B TSS & $.747^{*}$ & $.882^{*}$ & $.841^{*}$ & 1 \\
\hline
\end{tabular}

${ }^{*}$ Correlation is significant at the .01 level (two-tailed)

events, and brightness with positive events. In fact, the color black was very rarely associated with the positive emotion or positive emotion-laden words in the present study, and it was never the most common response given. The most common color response for the positive emotion words was yellow, and the most common response for the positive emotion-laden words was white.

The main goal of the present work was to provide researchers with a set of color norms to aid in stimulus construction and selection. The emotional Stroop paradigm is widely used to measure selective attention to emotional information. It is believed that the interference in this task is a result of the emotional content of the words presented (McKenna, 1986; McKenna \& Sharma, 1995). The present set of norms could be used to extend the work by Sutton and Altarriba (2008), in which they examined a "true Stroop effect" for emotion words. The norms can also be used with other paradigms, such as the affective Simon task or the RSVP paradigm.

The present work also has implications for the use of colors in experimental research. Often researchers use color to differentiate a target stimulus from other stimuli. For example, Keil and Ihssen (2004) examined the attentional blink effect for emotion words. Using the RSVP paradigm, they presented a series of neutral (e.g., to continue), positive (e.g., to admire), and negative (e.g., to destroy) verbs on the screen. The target verbs were presented in green, and the distractor verbs were presented in white. If different colors are associated with emotional stimuli, then color might actually act as a confounding variable if it is inherently associated with a specific emotion word or class of words.

Table 4 Total set size (TSS) and mean set size (MSS) for the positive emotion words for Sample A and Sample B

\begin{tabular}{lllll}
\hline & $\begin{array}{l}\text { Sample } \\
\text { A MSS }\end{array}$ & $\begin{array}{l}\text { Sample } \\
\text { B MSS }\end{array}$ & $\begin{array}{l}\text { Sample } \\
\text { A TSS }\end{array}$ & $\begin{array}{l}\text { Sample } \\
\text { B TSS }\end{array}$ \\
\hline Sample A MSS & 1 & $.803^{*}$ & $.687^{*}$ & $.563^{*}$ \\
Sample B MSS & $.803^{*}$ & 1 & $.712^{*}$ & $.746^{*}$ \\
Sample A TSS & $.687^{*}$ & $.712^{*}$ & 1 & $.832^{*}$ \\
Sample B TSS & $.563^{*}$ & $.746^{*}$ & $.832^{*}$ & 1 \\
\hline
\end{tabular}

${ }^{*}$ Correlation is significant at the .01 level (two-tailed)
Table 5 Total set size (TSS) and mean set size (MSS) for the positive emotion-laden words for Sample A and Sample B

\begin{tabular}{lllll}
\hline & $\begin{array}{l}\text { Sample } \\
\text { A MSS }\end{array}$ & $\begin{array}{l}\text { Sample } \\
\text { B MSS }\end{array}$ & $\begin{array}{l}\text { Sample } \\
\text { A TSS }\end{array}$ & $\begin{array}{l}\text { Sample } \\
\text { B TSS }\end{array}$ \\
\hline Sample A MSS & 1 & $.846^{*}$ & $.844^{*}$ & $.790^{*}$ \\
Sample B MSS & $.846^{*}$ & 1 & $.786^{*}$ & $.856^{*}$ \\
Sample A TSS & $.844^{*}$ & $.786^{*}$ & 1 & $.841^{*}$ \\
Sample B TSS & $.790^{*}$ & $.860^{*}$ & $.856^{*}$ & 1 \\
\hline
\end{tabular}

${ }^{*}$ Correlation is significant at the .01 level (two-tailed)

\section{Additional considerations}

It is important to note that the set of color norms provided was collected predominately from native English speakers. The associations between colors and words vary across cultures. For example, Hupka, Zaleski, Otto, Reidl, and Tarabrina (1997) examined the relationships between color and the following emotion words: (1)anger, (2)envy, (3) fear, and (4) jealousy. Participants from Germany, Mexico, Poland, Russia, and the United States were asked to indicate the extent to which one of these emotions reminded them of the colors black, blue, brown, gray, green, orange, pink, purple, red, violet, white, and yellow. The colors black and red were associated with anger in all countries. In addition, black and red were also associated with fear, except in Mexico. The word envy was associated with different colors in the various countries. For example, it was associated with green in the United States, yellow in Germany, and purple in Poland. The color association norms presented here should only be used with native English speakers in the United States.

In the present study, participants were asked to provide the first color that came to mind for each of the words presented. The variety of color responses was quite large across participants; however, it is important to note that research conducted by Van Overschelde, Rawson, and Dunlosky (2004) suggests that some color terms are more likely to be given than others. In their research, participants were given various category labels (e.g., colors, fruits, and weapons), and asked to generate as many responses as they could within $30 \mathrm{~s}$. For the "color" category, the colors blue and red were given most often as the first responses. The colors green, purple, yellow, and black were also given as first responses, but not as often. Other color words, including pink, brown, gold, and teal, were never given as first responses. It is possible that when one is asked to name a color for an object, such prototypical colors as red and blue are the first that come to mind, and reporting other colors takes more effort. In the present study, RED was the most common response for the negative words, but it was not the most common response for all of the word types. In fact, for the positive words, the most common response was YELLOW, and for the positive emotion-laden words, the most common response was WHITE. 
Research from the object identification and recognition literature has shown that color perception and color knowledge are distinct cognitive processes that can be distinguished from one another at the neuroanatomical level (e.g., Bartels \& Zeki, 2000; Bramão et al., 2012). The perception of surface color is a result of a bottom-up process generated by the color present in the object, whereas color knowledge is a component of the semantic information that individuals have stored about the object. In the present study, we asked participants to provide a color label for each emotional word. This represents the color knowledge that individuals had stored for emotional words, and not the perception of color. Research in this field has also indicated that color diagnosticity, the degree to which a color is representative of an object or is associated with an object, may influence how quickly an individual can correctly recognize an object in the environment (Tanaka \& Presnell, 1999). For example, a banana is an object that is high in color diagnosticity (i.e., associated with the color yellow), but a car is low in diagnosticity (there are many different colors for cars).

It is possible that some of the words in the present study may have been higher in color diagnosticity than others, particularly the concrete nouns in the emotion-laden lists. To determine whether the associations between the words and colors provided in the present study were the result of color diagnosticity, we examined the associative strength values provided in the University of South Florida (USF) free association norms database (Nelson, McEvoy, \& Schreiber, 1998). If a color is naturally symptomatic of a particular object, one would expect many individuals to provide this color response when presented with the object. Of our 55 negative emotion-laden words, 48 were available in the USF database. Of these 48 words, only seven were associated with any particular color. Also, the average association strength between the colors and words was low-.133, ranging from .014 to .338 . The word slave had the highest association strength, with the color black, which was the first of 18 possible responses given by participants. Likewise, 33 of the 41 positive emotion-laden words were available in the USF database. Of these 33 words, only eight were associated with a color word. Once again, the average association strength was low-.055, ranging from .014 to .183. A color response was never the first response given for any of the positive emotion-laden words. It seems unlikely that the degree of color diagnosticity was largely responsible for the colors reported for the majority of the items in the present study.

\section{Conclusions}

Color is pervasive in our everyday lives, and it plays an integral role in many aspects of human behavior, including object recognition (Ostergaard \& Davidoff, 1985; Wurm, Legge, Isenberg, \& Luebker, 1993), buying decisions (Bellizzi \& Hite, 1992), and communication (e.g., "once in a blue moon"). The present work adds to the literature suggesting that colors are associated with emotions. Theories of color describe how we experience, perceive, and categorize colors in terms of hue, chroma, saturation, values of brightness/lightness, and/or purpose (e.g., the Munsell Color System; for a review, see Indow, 1988). As we have illustrated in the present work, various aspects of color, such as brightness and hue, often become associated with specific emotions or with classes of emotional stimuli.

\section{Appendix 1}

Table 6 Color associations for the negative and positive emotion and emotion-laden words

\begin{tabular}{|c|c|c|c|}
\hline Word & Color & Freq & $\%$ \\
\hline \multicolumn{4}{|c|}{ Negative Emotion } \\
\hline \multicolumn{4}{|c|}{ Afraid } \\
\hline & Black & 50 & $50 \%$ \\
\hline & Red & 27 & $27 \%$ \\
\hline & Blue & 10 & $10 \%$ \\
\hline & Green & 3 & $3 \%$ \\
\hline & Brown & 2 & $2 \%$ \\
\hline & Gray & 2 & $2 \%$ \\
\hline & Yellow & 2 & $2 \%$ \\
\hline & Purple & 1 & $1 \%$ \\
\hline & Dark blue & 1 & $1 \%$ \\
\hline \multicolumn{4}{|c|}{ Agony } \\
\hline & Black & 49 & $49 \%$ \\
\hline & Red & 32 & $32 \%$ \\
\hline & Brown & 8 & $8 \%$ \\
\hline & Blue & 4 & $4 \%$ \\
\hline & Gray & 3 & $3 \%$ \\
\hline & Green & 2 & $2 \%$ \\
\hline & Orange & 1 & $1 \%$ \\
\hline & Silver & 1 & $1 \%$ \\
\hline & Teal & 1 & $1 \%$ \\
\hline \multicolumn{4}{|c|}{ Angry } \\
\hline & Red & 79 & $79 \%$ \\
\hline & Black & 14 & $14 \%$ \\
\hline & Brown & 2 & $2 \%$ \\
\hline & Blue & 2 & $2 \%$ \\
\hline & Yellow & 1 & $1 \%$ \\
\hline & Orange & 1 & $1 \%$ \\
\hline & Dark gray & 1 & $1 \%$ \\
\hline & Dusty red & 1 & $1 \%$ \\
\hline \multicolumn{4}{|c|}{ Anguished } \\
\hline & Red & 36 & $36 \%$ \\
\hline & Gray & 16 & $16 \%$ \\
\hline & Blue & 15 & $15 \%$ \\
\hline & Black & 13 & $13 \%$ \\
\hline & Green & 9 & $9 \%$ \\
\hline
\end{tabular}


Table 6 (continued)

\begin{tabular}{llll}
\hline Word & Color & Freq & $\%$ \\
\hline White & 3 & $3 \%$ \\
Yellow & 1 & $1 \%$ \\
Orange & 1 & $1 \%$ \\
Silver & 1 & $1 \%$ \\
& Fire Brick & 1 & $1 \%$ \\
& Red & & \\
& Black & 25 & $25 \%$ \\
Blue & 19 & $19 \%$ \\
Green & 16 & $16 \%$ \\
& Yellow & 13 & $13 \%$ \\
& Pink & 6 & $6 \%$ \\
White & 5 & $5 \%$ \\
& Gray & 5 & $5 \%$ \\
Orange & 4 & $4 \%$ \\
Brown & 4 & $4 \%$ \\
Dark Gray & 1 & $1 \%$ \\
Pale Green & 1 & $1 \%$ \\
Crimson & 1 & $1 \%$ \\
& & 1 & $1 \%$
\end{tabular}

Cruel

$\begin{array}{clll} & \text { Blue } & 43 & 43 \% \\ \text { Black } & 31 & 31 \% \\ \text { Gray } & 12 & 12 \% \\ \text { Red } & 7 & 7 \% \\ \text { Yellow } & 3 & 3 \% \\ \text { Brown } & \text { Green } & 2 & 2 \% \\ \text { Discouraged } & \text { Midnight blue } & 1 & 1 \% \\ & \text { Navy } & 1 & 1 \% \\ & \text { Blue } & & 1 \% \\ & \text { Black } & 29 & \\ \text { Red } & 29 \% \\ & \text { Gray } & 25 & 25 \% \\ & \text { Brown } & 15 & 15 \% \\ \text { Yellow } & 10 & 10 \% \\ \text { Green } & 9 & 9 \% \\ \text { Orange } & 5 & 5 \% \\ & & 3 & 3 \% \\ & 2 & 2 \%\end{array}$

Table 6 (continued)

\begin{tabular}{|c|c|c|c|}
\hline Word & Color & Freq & $\%$ \\
\hline & White & 1 & $1 \%$ \\
\hline & Indigo & 1 & $1 \%$ \\
\hline \multicolumn{4}{|c|}{ Disgusted } \\
\hline & Green & 25 & $25 \%$ \\
\hline & Brown & 21 & $21 \%$ \\
\hline & Red & 20 & $20 \%$ \\
\hline & Black & 18 & $18 \%$ \\
\hline & Gray & 4 & $4 \%$ \\
\hline & Blue & 3 & $3 \%$ \\
\hline & Orange & 3 & $3 \%$ \\
\hline & Purple & 2 & $2 \%$ \\
\hline & White & 1 & $1 \%$ \\
\hline & Yellow & 1 & $1 \%$ \\
\hline & Violet & 1 & $1 \%$ \\
\hline & Tan & 1 & $1 \%$ \\
\hline & Orange/red & 1 & $1 \%$ \\
\hline \multicolumn{4}{|c|}{ Dreadful } \\
\hline & Black & 54 & $53 \%$ \\
\hline & Red & 24 & $24 \%$ \\
\hline & Green & 7 & $7 \%$ \\
\hline & Gray & 6 & $6 \%$ \\
\hline & Blue & 5 & $5 \%$ \\
\hline & White & 2 & $2 \%$ \\
\hline & Brown & 1 & $1 \%$ \\
\hline & Dark red & 1 & $1 \%$ \\
\hline & Orange yellow & 1 & $1 \%$ \\
\hline \multicolumn{4}{|c|}{ Embarrassed } \\
\hline & Red & 67 & $66 \%$ \\
\hline & Blue & 8 & $8 \%$ \\
\hline & Green & 6 & $6 \%$ \\
\hline & Black & 5 & $5 \%$ \\
\hline & Yellow & 4 & $4 \%$ \\
\hline & Pink & 4 & $4 \%$ \\
\hline & Gray & 3 & $3 \%$ \\
\hline & Purple & 2 & $2 \%$ \\
\hline & Brown & 2 & $2 \%$ \\
\hline & Turquoise & 1 & $1 \%$ \\
\hline \multicolumn{4}{|c|}{ Enraged } \\
\hline & Red & 73 & $72 \%$ \\
\hline & Black & 15 & $15 \%$ \\
\hline & Blue & 4 & $4 \%$ \\
\hline & White & 1 & $1 \%$ \\
\hline & Gray & 1 & $1 \%$ \\
\hline & Yellow & 1 & $1 \%$ \\
\hline & Green & 1 & $1 \%$ \\
\hline & Purple & 1 & $1 \%$ \\
\hline & Orange & 1 & $1 \%$ \\
\hline & Gold & 1 & $1 \%$ \\
\hline & Silver & 1 & $1 \%$ \\
\hline
\end{tabular}


Table 6 (continued)

\begin{tabular}{|c|c|c|c|}
\hline Word & Color & Freq & $\%$ \\
\hline & Dark Red & 1 & $1 \%$ \\
\hline \multicolumn{4}{|c|}{ Fear } \\
\hline & Red & 50 & $49 \%$ \\
\hline & Black & 34 & $33 \%$ \\
\hline & Gray & 6 & $6 \%$ \\
\hline & Blue & 5 & $5 \%$ \\
\hline & Green & 4 & $4 \%$ \\
\hline & Yellow & 1 & $1 \%$ \\
\hline & Orange & 1 & $1 \%$ \\
\hline & Crimson & 1 & $1 \%$ \\
\hline \multicolumn{4}{|c|}{ Frustrated } \\
\hline & Red & 52 & $51 \%$ \\
\hline & Black & 17 & $17 \%$ \\
\hline & Green & 10 & $10 \%$ \\
\hline & Gray & 6 & $6 \%$ \\
\hline & Blue & 4 & $4 \%$ \\
\hline & Yellow & 4 & $4 \%$ \\
\hline & Purple & 3 & $3 \%$ \\
\hline & Orange & 3 & $3 \%$ \\
\hline & Brown & 2 & $2 \%$ \\
\hline \multicolumn{4}{|c|}{ Guilty } \\
\hline & Red & 30 & $30 \%$ \\
\hline & Green & 21 & $21 \%$ \\
\hline & Black & 16 & $16 \%$ \\
\hline & Gray & 12 & $12 \%$ \\
\hline & Blue & 11 & $11 \%$ \\
\hline & Yellow & 4 & $4 \%$ \\
\hline & White & 2 & $2 \%$ \\
\hline & Brown & 2 & $2 \%$ \\
\hline & Orange & 2 & $2 \%$ \\
\hline & Turquoise & 1 & $1 \%$ \\
\hline
\end{tabular}

Helpless

$\begin{array}{lll}\text { Black } & 24 & 24 \% \\ \text { Gray } & 17 & 17 \% \\ \text { White } & 14 & 14 \% \\ \text { Blue } & 13 & 13 \% \\ \text { Yellow } & 8 & 8 \% \\ \text { Red } & 7 & 75 \\ \text { Green } & 5 & 5 \% \\ \text { Brown } & 5 & 5 \% \\ \text { Orange } & 3 & 3 \% \\ \text { Purple } & 2 & 2 \% \\ \text { Tan } & 1 & 1 \% \\ \text { Coral } & 1 & 1 \% \\ \text { Light gray } & 1 & 1 \%\end{array}$

Hostile

$\begin{array}{lll}\text { Red } & 60 & 60 \% \\ \text { Black } & 26 & 26 \% \\ \text { White } & 3 & 3 \%\end{array}$

Table 6 (continued)

\begin{tabular}{llll}
\hline Word & Color & Freq & $\%$ \\
\hline \multirow{4}{*}{ Gray } & 3 & $3 \%$ \\
& Blue & 2 & $2 \%$ \\
Yellow & 1 & $1 \%$ \\
Green & 1 & $1 \%$ \\
& Purple & 1 & $1 \%$ \\
Bealousy & Brown & 1 & $1 \%$ \\
& Silver & 1 & $1 \%$ \\
& Dark red & 1 & $1 \%$ \\
& Green & 58 & $58 \%$
\end{tabular}

Red $22 \quad 22 \%$

Blue $99 \%$

Yellow $4 \quad 4 \%$

Purple $22 \%$

Black $22 \%$

Pink $11 \%$

Brown $\quad 1 \quad 1 \%$

Violet $\quad 1 \quad 1 \%$

Lonely

Blue

$46 \%$

Black $16 \quad 16 \%$

Gray $\quad 13 \quad 13 \%$

White $8 \quad 8 \%$

$\begin{array}{lll}\text { Red } & 4 & 4 \%\end{array}$

Green $3 \quad 3 \%$

Yellow $2 \%$

Pink $2 \%$

Purple $22 \%$

Light blue $\quad 1 \quad 1 \%$

Pale green $\quad 1 \quad 1 \%$

Mahogany $\quad 1 \quad 1 \%$

Blue violet $\quad 1 \quad 1 \%$

Blue gray $\quad 1 \quad 1 \%$

Mad

Red

$83 \%$

Black $\quad 13 \quad 13 \%$

Blue $11 \%$

Brown $\quad 1 \quad 1 \%$

Dark Red $11 \%$

Scarlett $\quad 1 \quad 1 \%$

Misery

$\begin{array}{lll}\text { Black } & 47 & 47 \% \\ \text { Red } & 20 & 20 \% \\ \text { Blue } & 11 & 11 \% \\ \text { Gray } & 10 & 10 \% \\ \text { Green } & 6 & 6 \% \\ \text { White } & 4 & 4 \% \\ \text { Purple } & 1 & 1 \% \\ \text { Brown } & 1 & 1 \%\end{array}$


Table 6 (continued)

\begin{tabular}{llll}
\hline Word & Color & Freq & $\%$ \\
\hline \multirow{3}{*}{ Moody } & Dark Purple & 1 & $1 \%$ \\
& & & \\
& Blue & 22 & $22 \%$ \\
Gray & 17 & $17 \%$ \\
Red & 13 & $13 \%$ \\
Black & 13 & $13 \%$ \\
Green & 9 & $9 \%$ \\
Purple & 8 & $8 \%$ \\
Brown & 8 & $8 \%$ \\
Yellow & 2 & $2 \%$ \\
Orange & 2 & $2 \%$ \\
Maroon & 2 & $2 \%$ \\
White & 1 & $1 \%$ \\
Pink & 1 & $1 \%$ \\
Navy & 1 & $1 \%$ \\
Rainbow & 1 & $1 \%$ \\
Indigo & 1 & $1 \%$ \\
& &
\end{tabular}

Nervous

$\begin{array}{lll}\text { Red } & 22 & 22 \% \\ \text { Green } & 16 & 16 \% \\ \text { Blue } & 15 & 15 \% \\ \text { Yellow } & 14 & 14 \% \\ \text { Gray } & 8 & 8 \% \\ \text { Orange } & 8 & 8 \% \\ \text { Black } & 5 & 5 \% \\ \text { Brown } & 5 & 5 \% \\ \text { White } & 4 & 4 \% \\ \text { Silver } & 1 & 1 \% \\ \text { Light green } & 1 & 1 \% \\ \text { Fuschia } & 1 & 1 \%\end{array}$

Panic

$\begin{array}{lll}\text { Red } & 72 & 71 \% \\ \text { Black } & 8 & 8 \% \\ \text { Blue } & 8 & 8 \% \\ \text { Yellow } & 4 & 4 \% \\ \text { White } & 2 & 2 \% \\ \text { Green } & 2 & 2 \% \\ \text { Purple } & 1 & 1 \% \\ \text { Brown } & 1 & 1 \% \\ \text { Orange } & 1 & 1 \% \\ \text { Beige } & 1 & 1 \% \\ \text { Indian red } & 1 & 1 \%\end{array}$

Rage
Table 6 (continued)

\begin{tabular}{llll}
\hline Word & Color & Freq & $\%$ \\
\hline Black & 28 & $28 \%$ \\
Blue & 16 & $16 \%$ \\
Green & 8 & $8 \%$ \\
Gray & 7 & $7 \%$ \\
Yellow & 6 & $6 \%$ \\
Purple & 3 & $3 \%$ \\
White & 1 & $1 \%$ \\
Pink & 1 & $1 \%$ \\
Brown & 1 & $1 \%$ \\
Orange & 1 & $1 \%$ \\
& Blue & & \\
Black & 61 & $60 \%$ \\
Red & 19 & $19 \%$ \\
Gray & 7 & $7 \%$ \\
Yellow & 4 & $4 \%$ \\
Green & 4 & $4 \%$ \\
Pink & 3 & $3 \%$ \\
Purple & 1 & $1 \%$ \\
Brown & 1 & $1 \%$ \\
Midnight blue & 1 & $1 \%$ \\
& & & \\
& & 1 &
\end{tabular}

Red

Black

$38 \quad 38 \%$

Blue

Yellow

White

Green

Gray

Orange

Purple

Brown

Shamed

Red

Blue

Gray

Black

Yellow

Green

Purple

Brown

Orange

White

Pink

Tan

Dark gray

Coffee black

Suspicious

Red

36

$36 \%$ 
Table 6 (continued)

\begin{tabular}{llll}
\hline Word & Color & Freq & $\%$ \\
\hline Green & 15 & $15 \%$ \\
Black & 14 & $14 \%$ \\
Gray & 13 & $13 \%$ \\
Blue & 8 & $8 \%$ \\
Orange & 7 & $7 \%$ \\
Purple & 3 & $3 \%$ \\
White & 2 & $2 \%$ \\
Yellow & 1 & $1 \%$ \\
Brown & 1 & $1 \%$ \\
Dark orange & 1 & $1 \%$
\end{tabular}

Tense

$\begin{array}{lll}\text { Red } & 42 & 42 \% \\ \text { Black } & 18 & 18 \% \\ \text { Green } & 9 & 9 \% \\ \text { Gray } & 7 & 7 \% \\ \text { Blue } & 6 & 6 \% \\ \text { Orange } & 6 & 6 \% \\ \text { Brown } & 4 & 4 \% \\ \text { White } & 3 & 3 \% \\ \text { Yellow } & 2 & 2 \% \\ \text { Purple } & 2 & 2 \% \\ \text { Tan } & 1 & 1 \% \\ \text { Navy } & 1 & 1 \%\end{array}$

Terrified

Troubled

$\begin{array}{lll}\text { Red } & 38 & 38 \% \\ \text { Black } & 36 & 36 \% \\ \text { Blue } & 9 & 9 \% \\ \text { Gray } & 6 & 6 \% \\ \text { Green } & 6 & 6 \% \\ \text { White } & 3 & 3 \% \\ \text { Yellow } & 1 & 1 \% \\ \text { Orange } & 1 & 1 \% \\ \text { Deep purple } & 1 & 1 \%\end{array}$

$\begin{array}{ll}\text { Red } & 32 \\ \text { Blue } & 17 \\ \text { Gray } & 13 \\ \text { Green } & 13 \\ \text { Black } & 13 \\ \text { Brown } & 5 \\ \text { Orange } & 2 \\ \text { White } & 1 \\ \text { Yellow } & 1 \\ \text { Pink } & 1 \\ \text { Purple } & 1 \\ \text { Silver } & 1 \\ \text { Dark gray } & 1\end{array}$

Unhappy
Table 6 (continued)

\begin{tabular}{|c|c|c|c|}
\hline Word & Color & Freq & $\%$ \\
\hline & Black & 19 & $19 \%$ \\
\hline & Gray & 15 & $15 \%$ \\
\hline & Red & 11 & $11 \%$ \\
\hline & Yellow & 4 & $4 \%$ \\
\hline & Brown & 4 & $4 \%$ \\
\hline & Green & 2 & $2 \%$ \\
\hline & Orange & 2 & $2 \%$ \\
\hline & White & 1 & $1 \%$ \\
\hline & Purple & 1 & $1 \%$ \\
\hline \multicolumn{4}{|c|}{ Upset } \\
\hline & Blue & 41 & $41 \%$ \\
\hline & Red & 27 & $27 \%$ \\
\hline & Black & 14 & $14 \%$ \\
\hline & Gray & 8 & $8 \%$ \\
\hline & Brown & 3 & $3 \%$ \\
\hline & Yellow & 1 & $1 \%$ \\
\hline & Green & 1 & $1 \%$ \\
\hline & Purple & 1 & $1 \%$ \\
\hline & Orange & 1 & $1 \%$ \\
\hline & Dark Red & 1 & $1 \%$ \\
\hline & Peach & 1 & $1 \%$ \\
\hline & Navy & 1 & $1 \%$ \\
\hline
\end{tabular}

$\begin{array}{lll}\text { Black } & 44 & 44 \% \\ \text { Red } & 23 & 23 \% \\ \text { Green } & 10 & 10 \% \\ \text { Yellow } & 6 & 6 \% \\ \text { Blue } & 5 & 5 \% \\ \text { Gray } & 3 & 3 \% \\ \text { Brown } & 3 & 3 \% \\ \text { Orange } & 3 & 3 \% \\ \text { Pink } & 1 & 1 \% \\ \text { Silver } & 1 & 1 \% \\ \text { Dark red } & 1 & 1 \%\end{array}$

Abortion

$\begin{array}{lll}\text { Red } & 51 & 50 \% \\ \text { Black } & 25 & 25 \% \\ \text { Blue } & 6 & 6 \% \\ \text { White } & 5 & 5 \% \\ \text { Gray } & 4 & 4 \% \\ \text { Yellow } & 4 & 4 \% \\ \text { Pink } & 3 & 3 \% \\ \text { Green } & 2 & 2 \% \\ \text { Brown } & 1 & 1 \% \\ \text { Dark red } & 1 & 1 \% \\ & & \\ \text { Red } & 48 & 48 \% \\ \text { Black } & 39 & 39 \%\end{array}$


Table 6 (continued)

\begin{tabular}{llll}
\hline Word & Color & Freq & $\%$ \\
\hline Blue & 4 & $4 \%$ \\
Gray & 4 & $4 \%$ \\
Green & 2 & $2 \%$ \\
White & 1 & $1 \%$ \\
Purple & 1 & $1 \%$ \\
Brown & 1 & $1 \%$
\end{tabular}

Bees

Yellow $\quad 99 \quad 99 \%$

Bloody

Black

Red

Blue

Black

Crimson

Wine red

Burial

Black

Brown

Gray

Red

White

Dark brown

Cancer

$\begin{array}{lll}\text { Black } & 34 & 33 \% \\ \text { Pink } & 21 & 20 \% \\ \text { Red } & 20 & 19 \% \\ \text { White } & 7 & 7 \% \\ \text { Yellow } & 5 & 5 \% \\ \text { Brown } & 5 & 5 \% \\ \text { Blue } & 4 & 4 \% \\ \text { Gray } & 3 & 3 \% \\ \text { Orange } & 2 & 2 \% \\ \text { Green } & 1 & 1 \% \\ \text { Tan } & 1 & 1 \%\end{array}$

Cemetery

$\begin{array}{llll} & \text { Black } & 55 & 54 \% \\ \text { Gray } & 33 & 33 \% \\ \text { White } & 6 & 6 \% \\ \text { Green } & 2 & 2 \% \\ \text { Brown } & 2 & 2 \% \\ \text { Red } & 1 & 1 \% \\ \text { Coffin } & \text { Silver } & 1 & 1 \% \\ & \text { Forest green } & 1 & 1 \% \\ & & & \\ & \text { Brown } & 53 & 53 \% \\ & \text { Black } & 39 & 39 \% \\ \text { Gray } & 2 & 2 \% \\ \text { Orange } & 1 & 1 \%\end{array}$

Table 6 (continued)

\begin{tabular}{llll}
\hline Word & Color & Freq & $\%$ \\
\hline \multirow{4}{*}{ Silver } & 1 & $1 \%$ \\
Tan & 1 & $1 \%$ \\
Magenta & 1 & $1 \%$ \\
Mahogany & 1 & $1 \%$ \\
& Crème/cream & 1 & $1 \%$ \\
& Black & & \\
White & 50 & $50 \%$ \\
Gray & 19 & $19 \%$ \\
Brown & 14 & $14 \%$ \\
Blue & 6 & $6 \%$ \\
Red & 5 & $5 \%$ \\
Yellow & 5 & $5 \%$ \\
Pink & 1 & $1 \%$ \\
White & 1 & $1 \%$ \\
& & 1 & $1 \%$
\end{tabular}

Crash

$\begin{array}{lll}\text { Red } & 53 & 52 \% \\ \text { Black } & 26 & 26 \% \\ \text { Gray } & 7 & 7 \% \\ \text { Blue } & 5 & 5 \% \\ \text { Brown } & 3 & 3 \% \\ \text { Green } & 3 & 3 \% \\ \text { Yellow } & 2 & 2 \% \\ \text { Purple } & 1 & 1 \% \\ \text { Orange } & 1 & 1 \% \\ & & \\ \text { Black } & 56 & 56 \% \\ \text { Orange } & 12 & 12 \% \\ \text { Red } & 11 & 11 \% \\ \text { Gray } & 9 & 9 \% \\ \text { White } & 4 & 4 \% \\ \text { Brown } & 4 & 4 \% \\ \text { Green } & 2 & 2 \% \\ \text { Blue } & 1 & 1 \% \\ \text { Pink } & 1 & 1 \%\end{array}$

Crisis

$\begin{array}{lll}\text { Red } & 59 & 58 \% \\ \text { Black } & 19 & 19 \% \\ \text { Blue } & 7 & 7 \% \\ \text { Gray } & 7 & 7 \% \\ \text { Yellow } & 2 & 2 \% \\ \text { Green } & 2 & 2 \% \\ \text { Orange } & 2 & 2 \% \\ \text { Brown } & 1 & 1 \% \\ \text { Beige } & 1 & 1 \% \\ \text { Dark red } & 1 & 1 \%\end{array}$

Danger

Red

83

$83 \%$ 
Table 6 (continued)

\begin{tabular}{llll}
\hline Word & Color & Freq & $\%$ \\
\hline Black & 9 & $9 \%$ \\
Orange & 4 & $4 \%$ \\
Yellow & 3 & $3 \%$ \\
Dark red & 1 & $1 \%$
\end{tabular}

Dead

$\begin{array}{lll}\text { Black } & 77 & 76 \% \\ \text { Red } & 12 & 12 \% \\ \text { White } & 3 & 3 \% \\ \text { Blue } & 2 & 2 \% \\ \text { Gray } & 2 & 2 \% \\ \text { Green } & 2 & 2 \% \\ \text { Brown } & 2 & 2 \% \\ \text { Purple } & 1 & 1 \%\end{array}$

Death

$\begin{array}{lll}\text { Black } & 86 & 86 \% \\ \text { Red } & 11 & 11 \% \\ \text { White } & 1 & 1 \% \\ \text { Gray } & 1 & 1 \% \\ \text { Orange } & 1 & 1 \%\end{array}$

Demon

$\begin{array}{llll}\text { Red } & 66 & 66 \% & \\ \text { Black } & 28 & 28 \% & \text { Illness } \\ \text { Blue } & 1 & 1 \% & \\ \text { Gray } & 1 & 1 \% & \\ \text { Green } & 1 & 1 \% & \\ \text { Purple } & 1 & 1 \% & \\ \text { Brown } & 1 & 1 \% & \end{array}$

Disaster

Divorce

$\begin{array}{lll}\text { Black } & 40 & 40 \% \\ \text { Red } & 31 & 31 \% \\ \text { Gray } & 11 & 11 \% \\ \text { Brown } & 9 & 9 \% \\ \text { Blue } & 6 & 6 \% \\ \text { Green } & 1 & 1 \% \\ \text { Maroon } & 1 & 1 \% \\ \text { Dark gray } & 1 & 1 \%\end{array}$

$\begin{array}{lll}\text { Black } & 47 & 47 \% \\ \text { Red } & 26 & 26 \% \\ \text { Blue } & 11 & 11 \% \\ \text { Green } & 7 & 7 \% \\ \text { Gray } & 5 & 5 \% \\ \text { Brown } & 3 & 3 \% \\ \text { White } & 1 & 1 \% \\ \text { Dark brown } & 1 & 1 \%\end{array}$

Funeral

Black

Gray

83

7

$82 \%$

$7 \%$
Table 6 (continued)

\begin{tabular}{llll}
\hline Word & Color & Freq & $\%$ \\
\hline & Brown & 7 & $7 \%$ \\
White & 2 & $2 \%$ \\
Red & 1 & $1 \%$ \\
Navy & 1 & $1 \%$
\end{tabular}

Gun

$\begin{array}{lll}\text { Black } & 74 & 74 \% \\ \text { Silver } & 11 & 11 \% \\ \text { Gray } & 8 & 8 \% \\ \text { Brown } & 3 & 3 \% \\ \text { Pink } & 2 & 2 \% \\ \text { Blue } & 1 & 1 \% \\ \text { Red } & 1 & 1 \%\end{array}$

Hell

Re

$80 \%$

Black $\quad 13 \quad 13 \%$

White $2 \%$

Blue $\quad 1 \quad 1 \%$

Gray $\quad 1 \quad 1 \%$

Green $\quad 1 \quad 1 \%$

Orange $\quad 1 \quad 1 \%$

$\begin{array}{lll}\text { Dark red } & 1 & 1 \%\end{array}$

Green $\quad 43 \quad 43 \%$

Red $23 \quad 23 \%$

Black $13 \quad 13 \%$

Blue $99 \%$

$\begin{array}{lll}\text { Gray } & 5 & 5 \%\end{array}$

Yellow $\quad 4 \quad 4 \%$

White $\quad 1 \quad 1 \%$

Pink $11 \%$

Brown $\quad 1 \quad 1 \%$

Infection

Red $\quad 40 \quad 40 \%$

Green $\quad 24 \quad 24 \%$

Black $\quad 11 \quad 11 \%$

Brown $8 \quad 8 \%$

White $\quad 5 \quad 5 \%$

Yellow $\quad 5 \quad 5 \%$

Pink 3 3 $3 \%$

Gray $\quad 2 \quad 2 \%$

Blue $11 \%$

Dark magenta $\quad 1 \quad 1 \%$

$\begin{array}{lll}\text { Red } & 62 & 61 \%\end{array}$

Black $\quad 15 \quad 15 \%$

Blue $\quad 5 \quad 5 \%$

Brown $\quad 5 \quad 5 \%$

White $\quad 3 \quad 3 \%$ 
Table 6 (continued)

\begin{tabular}{llll}
\hline Word & Color & Freq & $\%$ \\
\hline Purple & 3 & $3 \%$ \\
Pink & 3 & $3 \%$ \\
Gray & 2 & $2 \%$ \\
Orange & 2 & $2 \%$ \\
Green & 1 & $1 \%$ \\
Dark red & 1 & $1 \%$
\end{tabular}

Insult

$\begin{array}{lll}\text { Red } & 30 & 29 \% \\ \text { Black } & 27 & 26 \% \\ \text { Green } & 14 & 14 \% \\ \text { Gray } & 8 & 8 \% \\ \text { Blue } & 7 & 7 \% \\ \text { Brown } & 4 & 4 \% \\ \text { Purple } & 3 & 3 \% \\ \text { Brown } & 3 & 3 \% \\ \text { White } & 2 & 2 \% \\ \text { Yellow } & 1 & 1 \% \\ \text { Silver } & 1 & 1 \% \\ \text { Tan } & 1 & 1 \% \\ \text { Magenta } & 1 & 1 \%\end{array}$

Jail

$\begin{array}{lll}\text { Gray } & 41 & 41 \% \\ \text { Black } & 31 & 31 \% \\ \text { Orange } & 11 & 11 \% \\ \text { Silver } & 11 & 115 \\ \text { Brown } & 3 & 3 \% \\ \text { Blue } & 1 & 1 \% \\ \text { Red } & 1 & 1 \% \\ \text { Black \& White } & 1 & 1 \%\end{array}$

Killer

$\begin{array}{lll}\text { Red } & 54 & 54 \% \\ \text { Black } & 36 & 36 \% \\ \text { White } & 3 & 3 \% \\ \text { Gray } & 2 & 2 \% \\ \text { Blue } & 1 & 1 \% \\ \text { Brown } & 1 & 1 \% \\ \text { Dark Red } & 1 & 1 \% \\ \text { Scarlett } & 1 & 1 \% \\ \text { Charcoal } & 1 & 1 \%\end{array}$

Lie

$\begin{array}{lll}\text { Black } & 29 & 29 \% \\ \text { Red } & 24 & 24 \% \\ \text { White } & 17 & 17 \% \\ \text { Green } & 14 & 14 \% \\ \text { Blue } & 8 & 8 \% \\ \text { Gray } & 3 & 3 \% \\ \text { Yellow } & 1 & 1 \% \\ \text { Purple } & 1 & 1 \%\end{array}$

Table 6 (continued)

\begin{tabular}{|c|c|c|c|}
\hline Word & Color & Freq & $\%$ \\
\hline & Brown & 1 & $1 \%$ \\
\hline & Orange & 1 & $1 \%$ \\
\hline & Maroon & 1 & $1 \%$ \\
\hline \multicolumn{4}{|c|}{ Massacre } \\
\hline & Red & 79 & $79 \%$ \\
\hline & Black & 13 & $13 \%$ \\
\hline & Blue & 3 & $3 \%$ \\
\hline & White & 1 & $1 \%$ \\
\hline & Green & 1 & $1 \%$ \\
\hline & Brown & 1 & $1 \%$ \\
\hline & Gold & 1 & $1 \%$ \\
\hline & Dark red & 1 & $1 \%$ \\
\hline \multicolumn{4}{|c|}{ Morbid } \\
\hline & Black & 67 & $67 \%$ \\
\hline & Red & 11 & $11 \%$ \\
\hline & Gray & 9 & $9 \%$ \\
\hline & White & 4 & $4 \%$ \\
\hline & Brown & 3 & $3 \%$ \\
\hline & Blue & 2 & $2 \%$ \\
\hline & Green & 2 & $2 \%$ \\
\hline & Yellow & 1 & $1 \%$ \\
\hline
\end{tabular}

$\begin{array}{lll}\text { Black } & 75 & 74 \% \\ \text { Red } & 12 & 12 \% \\ \text { Blue } & 5 & 5 \% \\ \text { Gray } & 3 & 3 \% \\ \text { Green } & 3 & 3 \% \\ \text { Purple } & 2 & 2 \% \\ \text { Brown } & 1 & 1 \%\end{array}$

Pain

$\begin{array}{lll}\text { Red } & 72 & 71 \% \\ \text { Black } & 8 & 8 \% \\ \text { Blue } & 6 & 6 \% \\ \text { Yellow } & 3 & 3 \% \\ \text { White } & 2 & 2 \% \\ \text { Gray } & 2 & 2 \% \\ \text { Green } & 2 & 2 \% \\ \text { Purple } & 1 & 1 \% \\ \text { Brown } & 1 & 1 \% \\ \text { Orange } & 1 & 1 \% \\ \text { Dark Red } & 1 & 1 \% \\ \text { Flesh/skin } & 1 & 1 \% \\ \text { Crème/cream } & 1 & 1 \% \\ & & 35 \% \\ \text { Black } & 35 & 27 \% \\ \text { Green } & 27 & 26 \% \\ \text { Red } & 26 & 3 \% \\ \text { Yellow } & 3 & \end{array}$


Table 6 (continued)

\begin{tabular}{llll}
\hline Word & Color & Freq & $\%$ \\
\hline White & 2 & $2 \%$ \\
Blue & 2 & $2 \%$ \\
Clear & 2 & 25 \\
Purple & 2 & $2 \%$ \\
Gray & 1 & $1 \%$ \\
Lime green & 1 & $1 \%$
\end{tabular}

Pollute

$\begin{array}{lll}\text { Gray } & 38 & 38 \% \\ \text { Black } & 36 & 36 \% \\ \text { Green } & 10 & 10 \% \\ \text { Brown } & 10 & 10 \% \\ \text { White } & 1 & 1 \% \\ \text { Blue } & 1 & 1 \% \\ \text { Red } & 1 & 1 \% \\ \text { Yellow } & 1 & 1 \% \\ \text { Dark gray } & 1 & 1 \%\end{array}$

Poverty

$\begin{array}{lll}\text { Black } & 33 & 33 \% \\ \text { Brown } & 24 & 24 \% \\ \text { Gray } & 17 & 17 \% \\ \text { Blue } & 8 & 8 \% \\ \text { Green } & 6 & 6 \% \\ \text { Red } & 4 & 4 \% \\ \text { Orange } & 3 & 3 \% \\ \text { Yellow } & 1 & 1 \% \\ \text { Pink } & 1 & 1 \% \\ \text { Gold } & 1 & 1 \% \\ \text { Silver } & 1 & 1 \% \\ \text { Crimson } & 1 & 1 \%\end{array}$

Punishment

$\begin{array}{lll}\text { Red } & 50 & 49 \% \\ \text { Black } & 33 & 32 \% \\ \text { Blue } & 5 & 5 \% \\ \text { Gray } & 3 & 3 \% \\ \text { White } & 2 & 2 \% \\ \text { Green } & 2 & 2 \% \\ \text { Purple } & 2 & 2 \% \\ \text { Brown } & 2 & 2 \% \\ \text { Yellow } & 1 & 1 \% \\ \text { Silver } & 1 & 1 \% \\ \text { Dark red } & 1 & 1 \%\end{array}$

Rape

$\begin{array}{lll}\text { Red } & 57 & 57 \% \\ \text { Black } & 37 & 37 \% \\ \text { Gray } & 2 & 2 \% \\ \text { White } & 1 & 1 \% \\ \text { Blue } & 1 & 1 \% \\ \text { Purple } & 1 & 1 \%\end{array}$

Table 6 (continued)

\begin{tabular}{|c|c|c|c|}
\hline Word & Color & Freq & $\%$ \\
\hline & Brown & 1 & $1 \%$ \\
\hline \multicolumn{4}{|c|}{ Robber } \\
\hline & Black & 83 & $83 \%$ \\
\hline & Red & 10 & $10 \%$ \\
\hline & Green & 2 & $2 \%$ \\
\hline & White & 1 & $1 \%$ \\
\hline & Blue & 1 & $1 \%$ \\
\hline & Gray & 1 & $1 \%$ \\
\hline & Brown & 1 & $1 \%$ \\
\hline & Beige & 1 & $1 \%$ \\
\hline \multicolumn{4}{|c|}{ Sick } \\
\hline & Green & 61 & $60 \%$ \\
\hline & Blue & 8 & $8 \%$ \\
\hline & Red & 7 & $7 \%$ \\
\hline & Brown & 6 & $6 \%$ \\
\hline & Gray & 5 & $5 \%$ \\
\hline & Black & 4 & $4 \%$ \\
\hline & White & 3 & $3 \%$ \\
\hline & Yellow & 3 & $3 \%$ \\
\hline & Orange & 3 & $3 \%$ \\
\hline & Tan & 1 & $1 \%$ \\
\hline & Pale green & 1 & $1 \%$ \\
\hline \multicolumn{4}{|c|}{ Sickness } \\
\hline & Green & 47 & $47 \%$ \\
\hline & Red & 18 & $18 \%$ \\
\hline & Black & 12 & $12 \%$ \\
\hline & Blue & 8 & $8 \%$ \\
\hline & Yellow & 6 & $6 \%$ \\
\hline & Brown & 5 & $5 \%$ \\
\hline & Gray & 2 & $2 \%$ \\
\hline & White & 1 & $1 \%$ \\
\hline & Beige & 1 & $1 \%$ \\
\hline & Dark blue & 1 & $1 \%$ \\
\hline & Pale green & 1 & $1 \%$ \\
\hline \multicolumn{4}{|c|}{ Slave } \\
\hline & Black & 69 & $68 \%$ \\
\hline & Brown & 12 & $12 \%$ \\
\hline & Red & 8 & $8 \%$ \\
\hline & Gray & 4 & $4 \%$ \\
\hline & Green & 3 & $3 \%$ \\
\hline & White & 2 & $2 \%$ \\
\hline & Yellow & 2 & $2 \%$ \\
\hline & Blue & 1 & $1 \%$ \\
\hline \multicolumn{4}{|c|}{ Suicide } \\
\hline & Red & 46 & $45 \%$ \\
\hline & Black & 43 & $43 \%$ \\
\hline & Blue & 5 & $5 \%$ \\
\hline & Gray & 3 & $3 \%$ \\
\hline
\end{tabular}


Table 6 (continued)

\begin{tabular}{llll}
\hline Word & Color & Freq & $\%$ \\
\hline & White & 2 & $2 \%$ \\
Green & 1 & $1 \%$ \\
Orange & 1 & $1 \%$
\end{tabular}

Tomb

$\begin{array}{lll}\text { Black } & 40 & 40 \% \\ \text { Gray } & 28 & 28 \% \\ \text { Brown } & 17 & 17 \% \\ \text { Yellow } & 4 & 4 \% \\ \text { Red } & 3 & 3 \% \\ \text { Gold } & 2 & 2 \% \\ \text { Beige } & 2 & 2 \% \\ \text { Blue } & 1 & 1 \% \\ \text { Green } & 1 & 1 \% \\ \text { Tan } & 1 & 1 \% \\ \text { Burlywood } & 1 & 1 \% \\ \text { Sandstone } & 1 & 1 \%\end{array}$

Toothache

$\begin{array}{lll}\text { White } & 47 & 46 \% \\ \text { Red } & 33 & 32 \% \\ \text { Black } & 8 & 8 \% \\ \text { Yellow } & 4 & 4 \% \\ \text { Gray } & 3 & 3 \% \\ \text { Blue } & 2 & 2 \% \\ \text { Green } & 1 & 1 \% \\ \text { Purple } & 1 & 1 \% \\ \text { Brown } & 1 & 1 \% \\ \text { Dark red } & 1 & 1 \% \\ \text { Pale yellow } & 1 & 1 \%\end{array}$

Tornado

$\begin{array}{lll}\text { Gray } & 50 & 49 \% \\ \text { Black } & 19 & 19 \% \\ \text { Blue } & 12 & 125 \\ \text { Brown } & 8 & 8 \% \\ \text { Red } & 6 & 6 \% \\ \text { White } & 2 & 2 \% \\ \text { Yellow } & 1 & 1 \% \\ \text { Green } & 1 & 1 \% \\ \text { Purple } & 1 & 1 \% \\ \text { Orange } & 1 & 1 \% \\ \text { Dark gray } & 1 & 1 \% \\ & & \\ \text { Red } & 36 & 36 \% \\ \text { Black } & 36 & 36 \% \\ \text { Blue } & 12 & 12 \% \\ \text { Gray } & 7 & 7 \% \\ \text { White } & 2 & 2 \% \\ \text { Yellow } & 2 & 2 \% \\ \text { Purple } & 1 & 1 \%\end{array}$

Table 6 (continued)

\begin{tabular}{llll}
\hline Word & Color & Freq & $\%$ \\
\hline Brown & 1 & $1 \%$ \\
Orange & 1 & $1 \%$ \\
Burgundy & 1 & $1 \%$
\end{tabular}

Trash

Black $\quad 49 \quad 49 \%$

Gray $\quad 16 \quad 16 \%$

Brown $\quad 13 \quad 13 \%$

White $\quad 10 \quad 10 \%$

$\begin{array}{lll}\text { Green } & 9 & 9 \%\end{array}$

Maroon $\quad 1 \quad 1 \%$

$\begin{array}{lll}\text { Dark green } & 1 & 1 \%\end{array}$

Trauma

Red $\quad 51 \quad 51 \%$

Black $\quad 17 \quad 17 \%$

White $8 \quad 8 \%$

Blue $8 \quad 8 \%$

Gray $\quad 8 \quad 8 \%$

Green $\quad 4 \quad 4 \%$

Purple $\quad 1 \quad 1 \%$

Orange $\quad 1 \quad 1 \%$

Dark blue $\quad 1 \quad 1 \%$

Navy $\quad 1 \quad 1 \%$

Ugly

Brown $\quad 35 \quad 35 \%$

Black $\quad 26 \quad 26 \%$

Gray $\quad 8 \quad 8 \%$

Green $\quad 8 \quad 8 \%$

White $4 \quad 4 \%$

Blue $\quad 4 \quad 4 \%$

Yellow $4 \quad 4 \%$

Purple $3 \quad 3 \%$

Orange $\quad 3 \quad 3 \%$

Red $22 \%$

Silver $\quad 1 \quad 1 \%$

Chartreuse $\quad 1 \quad 1 \%$

$\begin{array}{lll}\text { Fuchsia } & 1 & 1 \%\end{array}$

Ulcer

Red $\quad 33 \quad 33 \%$

Black $23 \quad 23 \%$

Brown $99 \%$

White $99 \%$

$\begin{array}{lll}\text { Yellow } & 6 & 6 \%\end{array}$

$\begin{array}{lll}\text { Pink } & 6 & 6 \%\end{array}$

Blue $4 \quad 4 \%$

Gray $\quad 4 \quad 4 \%$

Orange $22 \%$

Peach $2 \%$

Clear $\quad 1 \quad 1 \%$ 
Table 6 (continued)

\begin{tabular}{llll}
\hline Word & Color & Freq & $\%$ \\
\hline & Green & 1 & $1 \%$ \\
& Crimson & 1 & $1 \%$
\end{tabular}

Unfaithful

$\begin{array}{lll}\text { Black } & 36 & 35 \% \\ \text { Red } & 27 & 27 \% \\ \text { Blue } & 8 & 8 \% \\ \text { Green } & 6 & 6 \% \\ \text { Gray } & 5 & 5 \% \\ \text { Orange } & 5 & 5 \% \\ \text { Yellow } & 4 & 4 \% \\ \text { Brown } & 3 & 3 \% \\ \text { White } & 3 & 3 \% \\ \text { Pink } & 2 & 2 \% \\ \text { Purple } & 2 & 2 \% \\ \text { Fire brick } & 1 & 1 \%\end{array}$

Victim

Red

Black

White

Gray

Blue

Purple

Brown

Yellow

Pink

Violet

Magenta

Midnight blue

Indigo

Violent

Red

Black

Purple

Violet

Blue

War

$\begin{array}{lll}\text { Red } & 58 & 58 \% \\ \text { Black } & 11 & 11 \% \\ \text { Green } & 10 & 10 \% \\ \text { Brown } & 9 & 9 \% \\ \text { Gray } & 6 & 6 \% \\ \text { Blue } & 4 & 4 \% \\ \text { Dark red } & 1 & 1 \%\end{array}$

Positive Emotion

Admired

Red
Blue
Pink

43

14

14
$43 \%$

$14 \%$

$14 \%$

$14 \%$

Table 6 (continued)

\begin{tabular}{llll}
\hline Word & Color & Freq & $\%$ \\
\hline Yellow & 12 & $12 \%$ \\
Orange & 4 & $4 \%$ \\
White & 4 & $4 \%$ \\
Black & 2 & $2 \%$ \\
Gold & 2 & $2 \%$ \\
Green & 2 & $2 \%$ \\
Violet & 1 & $1 \%$ \\
Light cyan & 1 & $1 \%$ \\
Silver & 1 & $1 \%$ \\
Sky blue & 1 & $1 \%$ \\
& Red & & \\
Pink & 49 & $49 \%$ \\
Yellow & 28 & $28 \%$ \\
& Blue & 8 & $8 \%$ \\
Purple & 8 & $8 \%$ \\
White & 3 & $3 \%$ \\
Green & 2 & $2 \%$ \\
Light blue & 1 & $1 \%$ \\
Gold & 1 & $1 \%$ \\
& & 1 & $1 \%$
\end{tabular}

Aroused

Red

63

$63 \%$

Pink

Yellow

Blue

White

Green

Brown

Black

Orange

Hot pink

Blue-green

Astonished

Yellow

White

Red

Blue

Orange

Green

Black

Gray

Baby blue

Brown

Purple

Light blue

Milk white

Chartreuse

Cream/ivory 
Table 6 (continued)

\begin{tabular}{llll}
\hline Word & Color & Freq & $\%$ \\
\hline Beautiful & & & \\
& Pink & 36 & $35 \%$ \\
& Blue & 20 & $19 \%$ \\
Yellow & 9 & $9 \%$ \\
& Purple & 8 & $8 \%$ \\
White & 6 & $6 \%$ \\
& Red & 6 & $6 \%$ \\
& Black & 5 & $5 \%$ \\
& Green & 4 & $4 \%$ \\
Brown & 2 & $2 \%$ \\
Gold & 2 & $2 \%$ \\
& Sky Blue & 2 & $2 \%$ \\
& Orange & 1 & $1 \%$ \\
Silver & 1 & $1 \%$
\end{tabular}

Brave

Red

Blue

Gold

Yellow

Brown

Black

White

Green

Orange

Purple

Violet

Silver

Bronze

Cheer

$\begin{array}{lll}\text { Yellow } & 38 & 38 \% \\ \text { Red } & 24 & 24 \% \\ \text { Blue } & 11 & 11 \% \\ \text { Pink } & 8 & 8 \% \\ \text { Orange } & 6 & 6 \% \\ \text { White } & 5 & 5 \% \\ \text { Purple } & 4 & 4 \% \\ \text { Green } & 3 & 3 \% \\ \text { Maroon } & 1 & 1 \%\end{array}$

\section{Confident}

$\begin{array}{lll}\text { Red } & 32 & 31 \% \\ \text { Blue } & 26 & 25 \% \\ \text { Yellow } & 13 & 13 \% \\ \text { Purple } & 6 & 6 \% \\ \text { White } & 4 & 4 \% \\ \text { Pink } & 4 & 4 \% \\ \text { Green } & 4 & 4 \% \\ \text { Gold } & 4 & 4 \% \\ \text { Black } & 3 & 3 \% \\ \text { Orange } & 3 & 3 \%\end{array}$

Table 6 (continued)

\begin{tabular}{llll}
\hline Word & Color & Freq & $\%$ \\
\hline \multirow{3}{*}{ Elated } & Violet & 2 & $2 \%$ \\
& Turquoise & 1 & $1 \%$ \\
& Yellow & & \\
& White & 21 & $21 \%$ \\
& Blue & 21 & $21 \%$ \\
Red & 21 & $21 \%$ \\
Pink & 9 & $9 \%$ \\
Purple & 6 & $6 \%$ \\
Gray & 5 & $5 \%$ \\
Green & 5 & $5 \%$ \\
Orange & 5 & $5 \%$ \\
Brown & 2 & $2 \%$ \\
Black & 2 & $2 \%$ \\
Baby blue & 1 & $1 \%$ \\
& & 1 & $1 \%$
\end{tabular}

Friendly

$\begin{array}{lll}\text { Yellow } & 41 & 41 \% \\ \text { Blue } & 18 & 18 \% \\ \text { Pink } & 16 & 16 \% \\ \text { Red } & 11 & 11 \% \\ \text { Green } & 7 & 7 \% \\ \text { Purple } & 3 & 3 \% \\ \text { Orange } & 3 & 3 \% \\ \text { Peach } & 1 & 1 \%\end{array}$

Blue $\quad 22 \quad 22 \%$

Yellow $\quad 17 \quad 17 \%$

Green $\quad 15 \quad 15 \%$

$\begin{array}{lll}\text { White } & 8 & 8 \%\end{array}$

Purple $8 \quad 8 \%$

$\begin{array}{lll}\text { Red } & 7 & 7 \%\end{array}$

$\begin{array}{lll}\text { Pink } & 7 & 7 \%\end{array}$

Orange $\quad 6 \quad 6 \%$

Gray $\quad 5 \quad 5 \%$

Lilac $\quad 1 \quad 1 \%$

Beige $11 \%$

Cream $11 \%$

Coral $\quad 1 \quad 1 \%$

Lime green $\quad 1 \quad 1 \%$

$\begin{array}{lll}\text { Black } & 1 & 1 \%\end{array}$

$\begin{array}{lll}\text { Yellow } & 53 & 53 \% \\ \text { Red } & 17 & 17 \% \\ \text { Pink } & 11 & 11 \% \\ \text { Blue } & 8 & 8 \% \\ \text { Green } & 6 & 6 \% \\ \text { Purple } & 2 & 2 \% \\ \text { White } & 2 & 2 \% \\ \text { Bright red } & 1 & 1 \%\end{array}$


Table 6 (continued)

\begin{tabular}{|c|c|c|c|c|c|c|c|}
\hline Word & Color & Freq & $\%$ & Word & Color & Freq & $\%$ \\
\hline \multirow[t]{15}{*}{ Hopeful } & & & & & White & 6 & $6 \%$ \\
\hline & Blue & 29 & $29 \%$ & & Pink & 3 & $3 \%$ \\
\hline & White & 28 & $28 \%$ & & Green & 3 & $3 \%$ \\
\hline & Yellow & 18 & $18 \%$ & & Black & 1 & $1 \%$ \\
\hline & Pink & 9 & $9 \%$ & & Orange & 1 & $1 \%$ \\
\hline & Red & 5 & $5 \%$ & & Gold & 1 & $1 \%$ \\
\hline & Orange & 3 & $3 \%$ & & Periwinkle & 1 & $1 \%$ \\
\hline & Green & 1 & $1 \%$ & Kind & & & \\
\hline & Silver & 1 & $1 \%$ & & Yellow & 27 & $26 \%$ \\
\hline & Magenta & 1 & $1 \%$ & & Blue & 20 & $20 \%$ \\
\hline & Light blue & 1 & $1 \%$ & & Pink & 20 & $20 \%$ \\
\hline & Antique white & 1 & $1 \%$ & & Green & 13 & $13 \%$ \\
\hline & Periwinkle & 1 & $1 \%$ & & White & 8 & $8 \%$ \\
\hline & Pale yellow & 1 & $1 \%$ & & Red & 5 & $5 \%$ \\
\hline & Brown & 1 & $1 \%$ & & Purple & 4 & $4 \%$ \\
\hline \multirow[t]{18}{*}{ Inspired } & & & & & Orange & 2 & $2 \%$ \\
\hline & Yellow & 23 & $23 \%$ & & Gray & 1 & $1 \%$ \\
\hline & White & 19 & $19 \%$ & & Charcoal & 1 & $1 \%$ \\
\hline & Blue & 19 & $19 \%$ & & Sea foam green & 1 & $1 \%$ \\
\hline & Red & 15 & $15 \%$ & Love & & & \\
\hline & Pink & 5 & $5 \%$ & & Red & 77 & $76 \%$ \\
\hline & Purple & 5 & $5 \%$ & & Pink & 9 & $9 \%$ \\
\hline & Green & 4 & $4 \%$ & & White & 4 & $4 \%$ \\
\hline & Orange & 2 & $2 \%$ & & Blue & 4 & $4 \%$ \\
\hline & Lavender & 2 & $2 \%$ & & Yellow & 2 & $2 \%$ \\
\hline & Black & 1 & $1 \%$ & & Purple & 2 & $2 \%$ \\
\hline & Violet & 1 & $1 \%$ & & Green & 1 & $1 \%$ \\
\hline & Gold & 1 & $1 \%$ & & Orange & 1 & $1 \%$ \\
\hline & Light blue & 1 & $1 \%$ & & Gold & 1 & $1 \%$ \\
\hline & Peach & 1 & $1 \%$ & Lust & & & \\
\hline & Teal & 1 & $1 \%$ & & Red & 69 & $68 \%$ \\
\hline & Blue-green & 1 & $1 \%$ & & Pink & 8 & $8 \%$ \\
\hline & Baby blue & 1 & $1 \%$ & & Black & 8 & $8 \%$ \\
\hline \multirow[t]{12}{*}{ Joy } & & & & & Purple & 4 & $4 \%$ \\
\hline & Yellow & 27 & $27 \%$ & & Yellow & 3 & $3 \%$ \\
\hline & Red & 21 & $21 \%$ & & Orange & 2 & $2 \%$ \\
\hline & Pink & 13 & $13 \%$ & & Violet & 2 & $2 \%$ \\
\hline & Blue & 12 & $12 \%$ & & White & 1 & $1 \%$ \\
\hline & White & 9 & $9 \%$ & & Blue & 1 & $1 \%$ \\
\hline & Green & 7 & $7 \%$ & & Green & 1 & $1 \%$ \\
\hline & Purple & 4 & $4 \%$ & & Brown & 1 & $1 \%$ \\
\hline & Orange & 4 & $4 \%$ & & Dark red & 1 & $1 \%$ \\
\hline & Gold & 1 & $1 \%$ & Merry & & & \\
\hline & Silver & 1 & $1 \%$ & & Red & 65 & $65 \%$ \\
\hline & Turquoise & 1 & $1 \%$ & & Green & 13 & $13 \%$ \\
\hline \multirow[t]{4}{*}{ Joyful } & & & & & Yellow & 10 & $10 \%$ \\
\hline & Yellow & 37 & $37 \%$ & & Blue & 6 & $6 \%$ \\
\hline & Blue & 26 & $26 \%$ & & Pink & 2 & $2 \%$ \\
\hline & Red & 21 & $21 \%$ & & Gray & 1 & $1 \%$ \\
\hline
\end{tabular}

Table 6 (continued)

Yellow $\quad 27 \quad 26 \%$

$0 \%$

$20 \%$

$13 \%$

$\%$

$\%$

$\%$

$\%$

$\%$

$\%$

$\%$

$76 \%$

$\%$

$\%$

$\%$

$\%$

$68 \%$

$\%$

$\%$

$\%$

$\%$

$\%$

$\%$

$\%$

$\%$

$\%$

$\%$

$\%$

\%

\%

$6 \%$

$\%$

$\%$ 
Table 6 (continued)

\begin{tabular}{llll}
\hline Word & Color & Freq & $\%$ \\
\hline & Purple & 1 & $1 \%$ \\
Orange & 1 & $1 \%$ \\
Salmon & 1 & $1 \%$
\end{tabular}

Nice

$\begin{array}{lll}\text { Yellow } & 30 & 29 \% \\ \text { Blue } & 24 & 23 \% \\ \text { Pink } & 17 & 17 \% \\ \text { White } & 13 & 13 \% \\ \text { Red } & 5 & 5 \% \\ \text { Green } & 2 & 2 \% \\ \text { Purple } & 2 & 2 \% \\ \text { Black } & 2 & 2 \% \\ \text { Gold } & 2 & 2 \% \\ \text { Silver } & 1 & 1 \% \\ \text { Lilac } & 1 & 1 \% \\ \text { Light blue } & 1 & 1 \% \\ \text { Indigo } & 1 & 1 \%\end{array}$

Passion

$\begin{array}{ll}\text { Red } & 79 \\ \text { Pink } & 6 \\ \text { White } & 4 \\ \text { Blue } & 4 \\ \text { Yellow } & 3 \\ \text { Purple } & 2 \\ \text { Green } & 1 \\ \text { Black } & 1 \\ \text { Dark red } & 1 \\ \text { Scarlett } & 1\end{array}$

Proud

$\begin{array}{lll}\text { Blue } & 28 & 27 \% \\ \text { Red } & 21 & 21 \% \\ \text { Yellow } & 16 & 16 \% \\ \text { White } & 10 & 10 \% \\ \text { Purple } & 8 & 8 \% \\ \text { Orange } & 5 & 5 \% \\ \text { Green } & 4 & 4 \% \\ \text { Black } & 3 & 3 \% \\ \text { Pink } & 2 & 2 \% \\ \text { Gold } & 2 & 2 \% \\ \text { Beige } & 1 & 1 \% \\ \text { Peach } & 1 & 1 \% \\ \text { Misty rose } & 1 & 1 \%\end{array}$

Relaxed

$\begin{array}{lll}\text { Blue } & 43 & 42 \% \\ \text { White } & 14 & 14 \% \\ \text { Yellow } & 12 & 12 \% \\ \text { Pink } & 6 & 6 \% \\ \text { Green } & 6 & 6 \% \\ \text { Red } & 4 & 4 \%\end{array}$

Table 6 (continued)

\begin{tabular}{|c|c|c|c|}
\hline Word & Color & Freq & $\%$ \\
\hline & Purple & 3 & $3 \%$ \\
\hline & Light blue & 3 & $3 \%$ \\
\hline & Tan & 2 & $2 \%$ \\
\hline & Sky blue & 2 & $2 \%$ \\
\hline & Gray & 1 & $1 \%$ \\
\hline & Brown & 1 & $1 \%$ \\
\hline & Peach & 1 & $1 \%$ \\
\hline & Lavender & 1 & $1 \%$ \\
\hline & Off white & 1 & $1 \%$ \\
\hline & Forest green & 1 & $1 \%$ \\
\hline & Baby blue & 1 & $1 \%$ \\
\hline \multicolumn{4}{|l|}{ Safe } \\
\hline & White & 31 & $30 \%$ \\
\hline & Blue & 30 & $29 \%$ \\
\hline & Yellow & 10 & $10 \%$ \\
\hline & Green & 8 & $8 \%$ \\
\hline & Gray & 6 & $6 \%$ \\
\hline & Red & 5 & $5 \%$ \\
\hline & Black & 4 & $4 \%$ \\
\hline & Pink & 2 & $2 \%$ \\
\hline & Purple & 1 & $1 \%$ \\
\hline & Brown & 1 & $1 \%$ \\
\hline & Orange & 1 & $1 \%$ \\
\hline & Gold & 1 & $1 \%$ \\
\hline & Beige & 1 & $1 \%$ \\
\hline & Pale blue & 1 & $1 \%$ \\
\hline \multicolumn{4}{|c|}{ Satisfied } \\
\hline & Blue & 33 & $33 \%$ \\
\hline & Red & 18 & $18 \%$ \\
\hline & Yellow & 14 & $14 \%$ \\
\hline & White & 11 & $11 \%$ \\
\hline & Green & 9 & $9 \%$ \\
\hline & Pink & 7 & $7 \%$ \\
\hline & Orange & 4 & $4 \%$ \\
\hline & Purple & 1 & $1 \%$ \\
\hline & Black & 1 & $1 \%$ \\
\hline & Gold & 1 & $1 \%$ \\
\hline & Lime green & 1 & $1 \%$ \\
\hline \multicolumn{4}{|c|}{ Secure } \\
\hline & Blue & 27 & $27 \%$ \\
\hline & White & 23 & $23 \%$ \\
\hline & Red & 14 & $14 \%$ \\
\hline & Yellow & 9 & $9 \%$ \\
\hline & Green & 8 & $8 \%$ \\
\hline & Gray & 5 & $5 \%$ \\
\hline & Black & 3 & $3 \%$ \\
\hline & Gold & 3 & $3 \%$ \\
\hline & Silver & 2 & $2 \%$ \\
\hline & Pink & 1 & $1 \%$ \\
\hline
\end{tabular}


Table 6 (continued)

\begin{tabular}{llll}
\hline Word & Color & Freq & $\%$ \\
\hline Purple & 1 & $1 \%$ \\
Brown & 1 & $1 \%$ \\
Orange & 1 & $1 \%$ \\
Light blue & 1 & $1 \%$ \\
Salmon & 1 & $1 \%$ \\
Sapphire & 1 & $1 \%$
\end{tabular}

Surprised

Red
Yellow
White
Blue
Orange
Pink
Green
Purple
Black
Violet
Gold
Silver

Triumphant

Yellow

Red

Blue

Gold

White

Purple

Green

Black

Gray

Brown

Pink

Orange

Silver

Bronze

Positive Emotion-Laden

Achievement

$\begin{array}{lll}\text { Blue } & 27 & 27 \% \\ \text { Gold } & 27 & 27 \% \\ \text { Red } & 16 & 16 \% \\ \text { Yellow } & 16 & 16 \% \\ \text { Green } & 6 & 6 \% \\ \text { White } & 5 & 5 \% \\ \text { Orange } & 2 & 2 \% \\ \text { Purple } & 1 & 1 \%\end{array}$

Adorable

$\begin{array}{lll}\text { Pink } & 50 & 49 \% \\ \text { Red } & 22 & 22 \% \\ \text { Blue } & 12 & 12 \% \\ \text { Yellow } & 6 & 6 \%\end{array}$

Table 6 (continued)

\begin{tabular}{llll}
\hline Word & Color & Freq & $\%$ \\
\hline Green & 4 & $4 \%$ \\
White & 2 & $2 \%$ \\
Brown & 2 & $2 \%$ \\
Purple & 1 & $1 \%$ \\
Tan & 1 & $1 \%$ \\
Light blue & 1 & $1 \%$ \\
Lavender & 1 & $1 \%$
\end{tabular}

Baby

Pink $\quad 39 \quad 38 \%$

White $21 \quad 21 \%$

Blue $\quad 20 \quad 20 \%$

$\begin{array}{lll}\text { Yellow } & 7 & 7 \%\end{array}$

$\begin{array}{lll}\text { Peach } & 6 & 6 \%\end{array}$

Black $3 \quad 3 \%$

Red $22 \%$

Brown $22 \%$

Green $\quad 1 \quad 1 \%$

Dodger blue $\quad 11 \%$

Birthday

Blue

Pink 21

$24 \%$

Yellow $\quad 20 \quad 20 \%$

Red $\quad 16 \quad 16 \%$

$\begin{array}{lll}\text { White } & 7 & 7 \%\end{array}$

Green $\quad 5 \quad 5 \%$

Purple $4 \quad 4 \%$

Black $22 \%$

Orange $\quad 1 \quad 1 \%$

$\begin{array}{lll}\text { Magenta } & 1 & 1 \%\end{array}$

Bouquet

Red $\quad 47 \quad 47 \%$

Pink $22 \quad 22 \%$

White $8 \quad 8 \%$

Green $\quad 8 \quad 8 \%$

Yellow $\quad 6 \quad 6 \%$

Blue $\quad 5 \quad 5 \%$

Purple $33 \%$

Brown $11 \%$

Orange $11 \%$

Green $\quad 94 \quad 93 \%$

Gray $22 \%$

Black $2 \%$

White $11 \%$

Brown $11 \%$

Gold $\quad 11 \%$

Champion

Gold $\quad 37 \quad 37 \%$

Red $22 \quad 22 \%$ 
Table 6 (continued)

\begin{tabular}{llll}
\hline Word & Color & Freq & $\%$ \\
\hline Yellow & 22 & $22 \%$ \\
Blue & 12 & $12 \%$ \\
White & 4 & $4 \%$ \\
Purple & 1 & $1 \%$ \\
Black & 1 & $1 \%$ \\
Orange & 1 & $1 \%$ \\
Silver & 1 & $1 \%$
\end{tabular}

Church

$\begin{array}{lll}\text { White } & 61 & 61 \% \\ \text { Brown } & 16 & 16 \% \\ \text { Gray } & 6 & 6 \% \\ \text { Red } & 6 & 6 \% \\ \text { Black } & 4 & 4 \% \\ \text { Yellow } & 3 & 3 \% \\ \text { Purple } & 2 & 2 \% \\ \text { Pink } & 1 & 1 \% \\ \text { Gold } & 1 & 1 \%\end{array}$

Diamond

$\begin{array}{lll}\text { White } & 71 & 70 \% \\ \text { Silver } & 8 & 8 \% \\ \text { Clear } & 6 & 6 \% \\ \text { Blue } & 3 & 3 \% \\ \text { Yellow } & 3 & 3 \% \\ \text { Red } & 3 & 3 \% \\ \text { Gold } & 3 & 3 \% \\ \text { Black } & 2 & 2 \% \\ \text { Pink } & 1 & 1 \% \\ \text { Purple } & 1 & 1 \%\end{array}$

Diploma

$\begin{array}{lll}\text { White } & 68 & 68 \% \\ \text { Blue } & 9 & 9 \% \\ \text { Black } & 6 & 6 \% \\ \text { Red } & 3 & 3 \% \\ \text { Gold } & 3 & 3 \% \\ \text { Crème/cream } & 2 & 2 \% \\ \text { Gray } & 1 & 1 \% \\ \text { Yellow } & 1 & 1 \% \\ \text { Green } & 1 & 1 \% \\ \text { Purple } & 1 & 1 \% \\ \text { Silver } & 1 & 1 \% \\ \text { Tan } & 1 & 1 \% \\ \text { Copper } & 1 & 1 \% \\ \text { Lime green } & 1 & 1 \% \\ \text { White/gold } & 1 & 1 \% \\ & & 33 \% \\ \text { White } & 33 & 15 \% \\ \text { Blue } & 15 & 12 \% \\ \text { Pink } & 12 & 12 \% \\ \text { Gold } & 12 & \end{array}$

Table 6 (continued)

\begin{tabular}{|c|c|c|c|}
\hline Word & Color & Freq & $\%$ \\
\hline & Red & 9 & $9 \%$ \\
\hline & Silver & 9 & $9 \%$ \\
\hline & Yellow & 7 & $7 \%$ \\
\hline & Purple & 1 & $1 \%$ \\
\hline & Orange & 1 & $1 \%$ \\
\hline & Light blue & 1 & $1 \%$ \\
\hline \multicolumn{4}{|c|}{ Freedom } \\
\hline & White & 30 & $30 \%$ \\
\hline & Blue & 28 & $28 \%$ \\
\hline & Red & 17 & $17 \%$ \\
\hline & Yellow & 8 & $8 \%$ \\
\hline & Green & 7 & $7 \%$ \\
\hline & Black & 4 & $4 \%$ \\
\hline & Brown & 2 & $2 \%$ \\
\hline & Orange & 1 & $1 \%$ \\
\hline & Gold & 1 & $1 \%$ \\
\hline & Silver & 1 & $1 \%$ \\
\hline & Royal blue & 1 & $1 \%$ \\
\hline & Forest green & 1 & $1 \%$ \\
\hline \multicolumn{4}{|c|}{ Friend } \\
\hline & Yellow & 27 & $27 \%$ \\
\hline & Blue & 24 & $24 \%$ \\
\hline & Pink & 14 & $14 \%$ \\
\hline & Green & 9 & $9 \%$ \\
\hline & Red & 6 & $6 \%$ \\
\hline & Purple & 5 & $5 \%$ \\
\hline & White & 4 & $4 \%$ \\
\hline & Brown & 3 & $3 \%$ \\
\hline & Gray & 2 & $2 \%$ \\
\hline & Black & 2 & $2 \%$ \\
\hline & Orange & 2 & $2 \%$ \\
\hline & Gold & 1 & $1 \%$ \\
\hline & Dark green & 1 & $1 \%$ \\
\hline \multicolumn{4}{|l|}{ Gift } \\
\hline & Blue & 30 & $29 \%$ \\
\hline & Red & 22 & $22 \%$ \\
\hline & Green & 13 & $13 \%$ \\
\hline & Yellow & 8 & $8 \%$ \\
\hline & Pink & 7 & $7 \%$ \\
\hline & White & 5 & $5 \%$ \\
\hline & Purple & 5 & $5 \%$ \\
\hline & Gold & 5 & $5 \%$ \\
\hline & Orange & 3 & $3 \%$ \\
\hline & Silver & 2 & $2 \%$ \\
\hline & Black & 1 & $1 \%$ \\
\hline & Sapphire & 1 & $1 \%$ \\
\hline \multicolumn{4}{|c|}{ God } \\
\hline & White & 75 & $74 \%$ \\
\hline & Black & 7 & $7 \%$ \\
\hline
\end{tabular}


Table 6 (continued)

\begin{tabular}{clll}
\hline Word & Color & Freq & $\%$ \\
\hline Gold & 6 & $6 \%$ \\
Red & 3 & $3 \%$ \\
Blue & 2 & $2 \%$ \\
Green & 2 & $2 \%$ \\
Purple & 1 & $1 \%$ \\
Brown & 1 & $1 \%$ \\
Bronze & 1 & $1 \%$ \\
Navy & 1 & $1 \%$ \\
Gray & 1 & $1 \%$ \\
Yellow & 1 & $1 \%$
\end{tabular}

Grin

$\begin{array}{lll}\text { White } & 28 & 27 \% \\ \text { Yellow } & 21 & 21 \% \\ \text { Red } & 18 & 18 \% \\ \text { Green } & 11 & 11 \% \\ \text { Pink } & 8 & 8 \% \\ \text { Blue } & 5 & 5 \% \\ \text { Gray } & 3 & 3 \% \\ \text { Black } & 2 & 2 \% \\ \text { Orange } & 2 & 2 \% \\ \text { Purple } & 1 & 1 \% \\ \text { Brown } & 1 & 1 \% \\ \text { Tan } & 1 & 1 \% \\ \text { Peach } & 1 & 1 \%\end{array}$

Heaven

$\begin{array}{lll}\text { White } & 71 & 71 \% \\ \text { Blue } & 17 & 17 \% \\ \text { Yellow } & 4 & 4 \% \\ \text { Gold } & 3 & 3 \% \\ \text { Green } & 1 & 1 \% \\ \text { Black } & 1 & 1 \% \\ \text { Orange } & 1 & 1 \% \\ \text { Holiday } & 1 & 1 \% \\ \text { Black } \& \text { white } & 1 & 1 \% \\ \text { Red } & 45 & 45 \% \\ \text { Green } & 29 & 29 \% \\ \text { Yellow } & 9 & 9 \% \\ \text { White } & 6 & 6 \% \\ \text { Blue } & 6 & 6 \% \\ \text { Pink } & 2 & 2 \% \\ \text { Brown } & 2 & 25 \\ \text { Orange } & 1 & 1 \% \\ \text { Gold } & 1 & 1 \%\end{array}$

Jewel

$\begin{array}{lll}\text { Red } & 21 & 21 \% \\ \text { White } & 17 & 17 \% \\ \text { Blue } & 17 & 17 \% \\ \text { Gold } & 12 & 12 \% \\ \text { Green } & 8 & 8 \%\end{array}$

Table 6 (continued)

\begin{tabular}{llll}
\hline Word & Color & Freq & $\%$ \\
\hline Purple & 8 & $8 \%$ \\
Pink & 5 & $5 \%$ \\
Clear & 2 & $2 \%$ \\
Yellow & 2 & $2 \%$ \\
Orange & 2 & $2 \%$ \\
Silver & 2 & $2 \%$ \\
Gray & 1 & $1 \%$ \\
Lavender & 1 & $1 \%$ \\
Golden red & 1 & $1 \%$ \\
Sapphire & 1 & $1 \%$ \\
Ruby & 1 & $1 \%$
\end{tabular}

Joke

Yellow $\quad 25 \quad 25 \%$

Blue $\quad 20 \quad 20 \%$

Red $18 \quad 18 \%$

Green $\quad 15 \quad 15 \%$

Orange $8 \quad 8 \%$

Pink $6 \quad 6 \%$

White $3 \quad 3 \%$

Purple $\quad 2 \quad 2 \%$

Black $22 \%$

Magenta $\quad 1 \quad 1 \%$

$\begin{array}{lll}\text { Azure } & 1 & 1 \%\end{array}$

Kiss

$\begin{array}{lll}\text { Red } & 63 & 63 \% \\ \text { Pink } & 26 & 26 \% \\ \text { Blue } & 5 & 5 \% \\ \text { White } & 2 & 2 \% \\ \text { Yellow } & 1 & 1 \% \\ \text { Black } & 1 & 1 \% \\ \text { Brown } & 1 & 1 \% \\ \text { Light pink } & 1 & 1 \%\end{array}$

Laughter

Yellow $\quad 42 \quad 42 \%$

Red $\quad 16 \quad 16 \%$

Blue $\quad 15 \quad 15 \%$

Pink $99 \%$

White $8 \quad 8 \%$

Green $\quad 3 \quad 3 \%$

Purple $\quad 3 \quad 3 \%$

$\begin{array}{lll}\text { Orange } & 1 & 1 \%\end{array}$

Gold $\quad 1 \quad 1 \%$

Sky blue $\quad 1 \quad 1 \%$

Peach orange $\quad 1 \quad 1 \%$

Green $\quad 51 \quad 50 \%$

Yellow $22 \quad 22 \%$

Blue $\quad 7 \quad 7 \%$

Red $55 \%$ 
Table 6 (continued)

\begin{tabular}{llll}
\hline Word & Color & Freq & $\%$ \\
\hline White & 4 & $4 \%$ \\
Pink & 3 & $3 \%$ \\
Gold & 3 & $3 \%$ \\
Gray & 2 & $2 \%$ \\
Purple & 1 & $1 \%$ \\
Black & 1 & $1 \%$ \\
Orange & 1 & $1 \%$ \\
& Dark green & 1 & $1 \%$ \\
& & & \\
& Green & 85 & $84 \%$ \\
& White & 4 & $4 \%$ \\
& Blue & 4 & $4 \%$ \\
& Gold & 3 & $3 \%$ \\
& Red & 2 & $2 \%$ \\
& Purple & 1 & $1 \%$ \\
& Silver & 1 & $1 \%$ \\
Dark green & 1 & $1 \%$
\end{tabular}

Miracle

$\begin{array}{lll}\text { White } & 48 & 48 \% \\ \text { Blue } & 18 & 18 \% \\ \text { Yellow } & 9 & 9 \% \\ \text { Gold } & 9 & 9 \% \\ \text { Pink } & 5 & 5 \% \\ \text { Red } & 4 & 4 \% \\ \text { Green } & 2 & 2 \% \\ \text { Gray } & 1 & 1 \% \\ \text { Purple } & 1 & 1 \% \\ \text { Orange } & 1 & 1 \% \\ \text { Silver } & 1 & 1 \% \\ \text { Aqua } & 1 & 1 \% \\ \text { Off-white } & 1 & 1 \%\end{array}$

Money

\begin{tabular}{|c|c|c|c|}
\hline & Green & 98 & $98 \%$ \\
\hline & Brown & 1 & $1 \%$ \\
\hline & Indigo & 1 & $1 \%$ \\
\hline \multicolumn{4}{|l|}{ Outdoors } \\
\hline & Green & 65 & $64 \%$ \\
\hline & Blue & 20 & $20 \%$ \\
\hline & White & 5 & $5 \%$ \\
\hline & Yellow & 5 & $5 \%$ \\
\hline & Brown & 3 & $3 \%$ \\
\hline & Green & 65 & $64 \%$ \\
\hline & Blue & 20 & $20 \%$ \\
\hline & White & 5 & $5 \%$ \\
\hline & Yellow & 5 & $5 \%$ \\
\hline & Brown & 3 & $3 \%$ \\
\hline & Gray & 1 & $1 \%$ \\
\hline & Red & 1 & $1 \%$ \\
\hline & Sky blue & 1 & $1 \%$ \\
\hline
\end{tabular}

Table 6 (continued)

\begin{tabular}{|c|c|c|c|}
\hline Word & Color & Freq & $\%$ \\
\hline & Forest green & 1 & $1 \%$ \\
\hline \multicolumn{4}{|c|}{ Peace } \\
\hline & White & 44 & $44 \%$ \\
\hline & Blue & 28 & $28 \%$ \\
\hline & Yellow & 8 & $8 \%$ \\
\hline & Green & 8 & $8 \%$ \\
\hline & Pink & 4 & $4 \%$ \\
\hline & Red & 2 & $2 \%$ \\
\hline & Orange & 2 & $2 \%$ \\
\hline & Purple & 1 & $1 \%$ \\
\hline & Gold & 1 & $1 \%$ \\
\hline & Sky blue & 1 & $1 \%$ \\
\hline & Light blue & 1 & $1 \%$ \\
\hline \multicolumn{4}{|c|}{ Pretty } \\
\hline & Pink & 64 & $63 \%$ \\
\hline & Red & 9 & $9 \%$ \\
\hline & Blue & 6 & $6 \%$ \\
\hline & Black & 6 & $6 \%$ \\
\hline & Yellow & 5 & $5 \%$ \\
\hline & Purple & 5 & $5 \%$ \\
\hline & White & 4 & $4 \%$ \\
\hline & Green & 1 & $1 \%$ \\
\hline & Brown & 1 & $1 \%$ \\
\hline & Light pink & 1 & $1 \%$ \\
\hline \multicolumn{4}{|c|}{ Rescue } \\
\hline & Red & 62 & $61 \%$ \\
\hline & Blue & 15 & $15 \%$ \\
\hline & Yellow & 8 & $8 \%$ \\
\hline & White & 5 & $5 \%$ \\
\hline & Black & 3 & $3 \%$ \\
\hline & Green & 2 & $2 \%$ \\
\hline & Brown & 2 & $2 \%$ \\
\hline & Tan & 1 & $1 \%$ \\
\hline & Indigo & 1 & $1 \%$ \\
\hline \multicolumn{4}{|c|}{ Riches } \\
\hline & Green & 39 & $39 \%$ \\
\hline & Gold & 39 & $39 \%$ \\
\hline & Yellow & 11 & $11 \%$ \\
\hline & Red & 7 & $7 \%$ \\
\hline & Blue & 3 & $3 \%$ \\
\hline & White & 1 & $1 \%$ \\
\hline \multicolumn{4}{|c|}{ Romantic } \\
\hline & Red & 87 & $87 \%$ \\
\hline & Pink & 8 & $8 \%$ \\
\hline & White & 1 & $1 \%$ \\
\hline & Blue & 1 & $1 \%$ \\
\hline & Purple & 1 & $1 \%$ \\
\hline & Black & 1 & $1 \%$ \\
\hline & Wine red & 1 & $1 \%$ \\
\hline
\end{tabular}


Table 6 (continued)

\begin{tabular}{|c|c|c|c|}
\hline Word & Color & Freq & $\%$ \\
\hline \multicolumn{4}{|c|}{ Thrill } \\
\hline & Red & 51 & $51 \%$ \\
\hline & Blue & 14 & $14 \%$ \\
\hline & Yellow & 10 & $10 \%$ \\
\hline & Green & 8 & $8 \%$ \\
\hline & Orange & 7 & $7 \%$ \\
\hline & Black & 6 & $6 \%$ \\
\hline & White & 1 & $1 \%$ \\
\hline & Purple & 1 & $1 \%$ \\
\hline & Silver & 1 & $1 \%$ \\
\hline & Aqua & 1 & $1 \%$ \\
\hline & Forest green & 1 & $1 \%$ \\
\hline \multicolumn{4}{|c|}{ Treasure } \\
\hline & Gold & 73 & $73 \%$ \\
\hline & Brown & 9 & $9 \%$ \\
\hline & Yellow & 8 & $8 \%$ \\
\hline & Pink & 2 & $2 \%$ \\
\hline & Green & 2 & $2 \%$ \\
\hline & White & 1 & $1 \%$ \\
\hline & Blue & 1 & $1 \%$ \\
\hline & Red & 1 & $1 \%$ \\
\hline & Purple & 1 & $1 \%$ \\
\hline & Maroon & 1 & $1 \%$ \\
\hline & Pearl white & 1 & $1 \%$ \\
\hline
\end{tabular}

Triumph

Yellow
Gold
Red
Blue
White
Green
Black
Gray
Purple
Brown
Silver
Bronze
Indigo

Trophy

$\begin{array}{clll} & \text { Gold } & 74 & 73 \% \\ \text { Yellow } & 17 & 17 \% \\ \text { Silver } & 4 & 4 \% \\ \text { Green } & 2 & 2 \% \\ \text { Gray } & 1 & 1 \% \\ & \text { Red } & 1 & 1 \% \\ \text { Vacation } & \text { Black } & 1 & 1 \% \\ & \text { Bronze } & 1 & 1 \% \\ & & & \end{array}$

Table 6 (continued)

\begin{tabular}{|c|c|c|c|}
\hline Word & Color & Freq & $\%$ \\
\hline & Yellow & 28 & $27 \%$ \\
\hline & Red & 4 & $4 \%$ \\
\hline & Orange & 4 & $4 \%$ \\
\hline & White & 3 & $3 \%$ \\
\hline & Pink & 3 & $3 \%$ \\
\hline & Green & 3 & $3 \%$ \\
\hline & Purple & 2 & $2 \%$ \\
\hline & Tan & 2 & $2 \%$ \\
\hline & Beige & 2 & $2 \%$ \\
\hline & Black & 1 & $1 \%$ \\
\hline & Gold & 1 & $1 \%$ \\
\hline \multicolumn{4}{|c|}{ Victory } \\
\hline & Red & 31 & $31 \%$ \\
\hline & Gold & 22 & $22 \%$ \\
\hline & Blue & 20 & $20 \%$ \\
\hline & White & 10 & $10 \%$ \\
\hline & Yellow & 7 & $7 \%$ \\
\hline & Green & 4 & $4 \%$ \\
\hline & Silver & 2 & $2 \%$ \\
\hline & Pink & 1 & $1 \%$ \\
\hline & Black & 1 & $1 \%$ \\
\hline & Brown & 1 & $1 \%$ \\
\hline & Orange & 1 & $1 \%$ \\
\hline & Violet & 1 & $1 \%$ \\
\hline
\end{tabular}

Waterfall

$\begin{array}{lll}\text { Blue } & 79 & 79 \% \\ \text { White } & 12 & 12 \% \\ \text { Gray } & 3 & 3 \% \\ \text { Clear } & 2 & 2 \% \\ \text { Green } & 1 & 1 \% \\ \text { Aqua } & 1 & 1 \% \\ \text { Forest } & 1 & 1 \% \\ \text { Rainbow } & 1 & 1 \% \\ & & \\ \text { White } & 95 & 95 \% \\ \text { Red } & 2 & 2 \% \\ \text { Purple } & 1 & 1 \% \\ \text { Black } & 1 & 1 \% \\ \text { Gold } & 1 & 1 \%\end{array}$

Wish

$\begin{array}{lll}\text { White } & 34 & 33 \% \\ \text { Blue } & 24 & 23 \% \\ \text { Yellow } & 15 & 15 \% \\ \text { Green } & 8 & 8 \% \\ \text { Gold } & 7 & 7 \% \\ \text { Red } & 6 & 6 \% \\ \text { Pink } & 5 & 5 \% \\ \text { Purple } & 1 & 1 \% \\ \text { Orange } & 1 & 1 \%\end{array}$


Table 6 (continued)

\begin{tabular}{llll}
\hline Word & Color & Freq & $\%$ \\
\hline & Silver & 1 & $1 \%$ \\
Cream & 1 & $1 \%$ \\
\hline
\end{tabular}

\section{Appendix 2}

Table 7 Color associations for Sample A and Sample B for the negative and positive emotion and emotion-laden words

\begin{tabular}{|c|c|c|}
\hline Word & Sample A & Sample B \\
\hline \multicolumn{3}{|c|}{ Negative Emotion } \\
\hline \multirow[t]{9}{*}{ Afraid } & Black $53 \%$ & Black $49 \%$ \\
\hline & $\operatorname{Red} 24 \%$ & Red $31 \%$ \\
\hline & Blue $12 \%$ & Blue $8 \%$ \\
\hline & Green $2 \%$ & Green $4 \%$ \\
\hline & Brown $2 \%$ & Brown $2 \%$ \\
\hline & Yellow $4 \%$ & Gray $4 \%$ \\
\hline & Dark Blue $2 \%$ & Purple $2 \%$ \\
\hline & $\mathrm{TSS}=7$ & $\mathrm{TSS}=7$ \\
\hline & $\mathrm{MSS}=4$ & $\mathrm{MSS}=5$ \\
\hline \multirow[t]{9}{*}{ Agony } & Black $44 \%$ & Black $53 \%$ \\
\hline & Red $34 \%$ & $\operatorname{Red} 29 \%$ \\
\hline & Brown $10 \%$ & Brown $6 \%$ \\
\hline & Blue $4 \%$ & Blue $4 \%$ \\
\hline & Gray $4 \%$ & Gray $2 \%$ \\
\hline & Orange $2 \%$ & Green $4 \%$ \\
\hline & Silver $2 \%$ & Teal $2 \%$ \\
\hline & $\mathrm{TSS}=7$ & $\mathrm{TSS}=7$ \\
\hline & $\mathrm{MSS}=5$ & $\mathrm{MSS}=5$ \\
\hline \multirow[t]{8}{*}{ Angry } & Red $78 \%$ & Red $78 \%$ \\
\hline & Black $16 \%$ & Black $12 \%$ \\
\hline & Brown $2 \%$ & Brown $2 \%$ \\
\hline & Orange $2 \%$ & Blue $4 \%$ \\
\hline & Yellow $2 \%$ & Dark gray $2 \%$ \\
\hline & $\mathrm{TSS}=5$ & Dusty red $2 \%$ \\
\hline & $\mathrm{MSS}=2$ & $\mathrm{TSS}=6$ \\
\hline & & $\mathrm{MSS}=3$ \\
\hline \multirow[t]{10}{*}{ Anguished } & Red $40 \%$ & Red $35 \%$ \\
\hline & Gray $18 \%$ & Gray $15 \%$ \\
\hline & Blue $12 \%$ & Blue $20 \%$ \\
\hline & Black $12 \%$ & Black $15 \%$ \\
\hline & Green $10 \%$ & Green $9 \%$ \\
\hline & White $6 \%$ & Yellow $2 \%$ \\
\hline & Orange $2 \%$ & Silver $2 \%$ \\
\hline & $\mathrm{TSS}=7$ & Fire brick $2 \%$ \\
\hline & $\mathrm{MSS}=6$ & $\mathrm{TSS}=8$ \\
\hline & & $\mathrm{MSS}=5$ \\
\hline Contempt & Red $23 \%$ & Red $26 \%$ \\
\hline
\end{tabular}

Table 7 (continued)

\begin{tabular}{|c|c|c|}
\hline Word & Sample A & Sample B \\
\hline & Black $18 \%$ & Black $20 \%$ \\
\hline & Blue $20 \%$ & Blue $12 \%$ \\
\hline & Green $10 \%$ & Green $16 \%$ \\
\hline & Yellow $7 \%$ & Yellow $4 \%$ \\
\hline & Pink $4 \%$ & Pink $6 \%$ \\
\hline & White $4 \%$ & White $6 \%$ \\
\hline & Gray $6 \%$ & Gray $2 \%$ \\
\hline & Orange $4 \%$ & Orange $4 \%$ \\
\hline & Pale green $2 \%$ & Brown $2 \%$ \\
\hline & Crimson $2 \%$ & Dark gray $2 \%$ \\
\hline & $\mathrm{TSS}=11$ & $\mathrm{TSS}=11$ \\
\hline & $\mathrm{MSS}=7$ & $\mathrm{MSS}=5$ \\
\hline \multirow[t]{10}{*}{ Cruel } & $\operatorname{Red} 48 \%$ & Red $43 \%$ \\
\hline & Black $35 \%$ & Black $33 \%$ \\
\hline & Green $6 \%$ & Green $6 \%$ \\
\hline & Blue $2 \%$ & Blue $6 \%$ \\
\hline & Gray $4 \%$ & Gray $4 \%$ \\
\hline & White $4 \%$ & White $4 \%$ \\
\hline & Dark red $2 \%$ & Yellow $2 \%$ \\
\hline & $\mathrm{TSS}=7$ & Purple $2 \%$ \\
\hline & $\mathrm{MSS}=3$ & $\mathrm{TSS}=8$ \\
\hline & & $\mathrm{MSS}=4$ \\
\hline \multirow[t]{9}{*}{ Depressed } & Blue $39 \%$ & Blue $46 \%$ \\
\hline & Black $33 \%$ & Black $28 \%$ \\
\hline & Gray $12 \%$ & Gray $12 \%$ \\
\hline & Red $8 \%$ & Red $6 \%$ \\
\hline & Yellow $2 \%$ & Yellow $4 \%$ \\
\hline & Green $2 \%$ & Midnight blue $2 \%$ \\
\hline & Brown $4 \%$ & Navy $2 \%$ \\
\hline & $\mathrm{TSS}=7$ & $\mathrm{TSS}=7$ \\
\hline & $\mathrm{MSS}=3$ & $\mathrm{MSS}=3$ \\
\hline \multirow[t]{11}{*}{ Discouraged } & Blue $27 \%$ & Blue $31 \%$ \\
\hline & Black $29 \%$ & Black $21 \%$ \\
\hline & Red $12 \%$ & Red $18 \%$ \\
\hline & Gray $12 \%$ & Gray $8 \%$ \\
\hline & Brown $10 \%$ & Brown $8 \%$ \\
\hline & Yellow $4 \%$ & Yellow $6 \%$ \\
\hline & Green $2 \%$ & Green $4 \%$ \\
\hline & Orange $2 \%$ & White $2 \%$ \\
\hline & $\mathrm{TSS}=8$ & Indigo $2 \%$ \\
\hline & $\mathrm{MSS}=4$ & $\mathrm{TSS}=9$ \\
\hline & & $\mathrm{MSS}=5$ \\
\hline \multirow[t]{7}{*}{ Disgusted } & Green $24 \%$ & Green $26 \%$ \\
\hline & Brown $18 \%$ & Brown $24 \%$ \\
\hline & Red $16 \%$ & Red $24 \%$ \\
\hline & Black $18 \%$ & Black $18 \%$ \\
\hline & Gray $6 \%$ & Gray $2 \%$ \\
\hline & Blue $2 \%$ & Blue $4 \%$ \\
\hline & Purple $2 \%$ & Purple $2 \%$ \\
\hline
\end{tabular}


Table 7 (continued)

\begin{tabular}{|c|c|c|}
\hline Word & Sample A & Sample B \\
\hline & Orange $6 \%$ & Orange/red $2 \%$ \\
\hline & Yellow $2 \%$ & $\mathrm{TSS}=8$ \\
\hline & $\operatorname{Tan} 2 \%$ & $\mathrm{MSS}=5$ \\
\hline & Violet $2 \%$ & \\
\hline & White $2 \%$ & \\
\hline & $\mathrm{TSS}=12$ & \\
\hline & $\mathrm{MSS}=6$ & \\
\hline \multirow[t]{10}{*}{ Dreadful } & Black $53 \%$ & Black $54 \%$ \\
\hline & Red $20 \%$ & Red $27 \%$ \\
\hline & Green $8 \%$ & Green $6 \%$ \\
\hline & Gray $8 \%$ & Gray $4 \%$ \\
\hline & Blue $4 \%$ & Blue $6 \%$ \\
\hline & White $2 \%$ & White $2 \%$ \\
\hline & Brown $2 \%$ & Dark red $2 \%$ \\
\hline & Orange/yellow $2 \%$ & $\mathrm{TSS}=7$ \\
\hline & $\mathrm{TSS}=8$ & $\mathrm{MSS}=4$ \\
\hline & $\mathrm{MSS}=5$ & \\
\hline \multirow[t]{12}{*}{ Embarrassed } & Red $64 \%$ & Red $67 \%$ \\
\hline & Blue $10 \%$ & Blue $6 \%$ \\
\hline & Green $8 \%$ & Green $4 \%$ \\
\hline & Black $4 \%$ & Black $6 \%$ \\
\hline & Yellow 6 \% & Yellow $2 \%$ \\
\hline & Pink $4 \%$ & Pink $4 \%$ \\
\hline & Gray $2 \%$ & Gray $4 \%$ \\
\hline & Purple $2 \%$ & Purple $2 \%$ \\
\hline & $\mathrm{TSS}=8$ & Brown $4 \%$ \\
\hline & $\mathrm{MSS}=6$ & Turquoise $2 \%$ \\
\hline & & $\mathrm{TSS}=10$ \\
\hline & & $\mathrm{MSS}=7$ \\
\hline \multirow[t]{10}{*}{ Enraged } & $\operatorname{Red} 72 \%$ & $\operatorname{Red} 73 \%$ \\
\hline & Black $13 \%$ & Black $17 \%$ \\
\hline & Blue $6 \%$ & Blue $2 \%$ \\
\hline & White $2 \%$ & Gray $2 \%$ \\
\hline & Yellow $2 \%$ & Orange $2 \%$ \\
\hline & Green $2 \%$ & Gold $2 \%$ \\
\hline & Purple $2 \%$ & Dark red $2 \%$ \\
\hline & Silver $2 \%$ & $\mathrm{TSS}=7$ \\
\hline & $\mathrm{TSS}=8$ & $\mathrm{MSS}=2$ \\
\hline & $\mathrm{MSS}=3$ & \\
\hline \multirow[t]{9}{*}{ Fear } & $\operatorname{Red} 54 \%$ & $\operatorname{Red} 44 \%$ \\
\hline & Black $32 \%$ & Black $35 \%$ \\
\hline & Gray $4 \%$ & Gray $8 \%$ \\
\hline & Blue $6 \%$ & Blue $4 \%$ \\
\hline & Green $2 \%$ & Green $6 \%$ \\
\hline & Yellow $2 \%$ & Orange $2 \%$ \\
\hline & $\mathrm{TSS}=6$ & Crimson $2 \%$ \\
\hline & $\mathrm{MSS}=4$ & $\mathrm{TSS}=7$ \\
\hline & & $\mathrm{MSS}=5$ \\
\hline Frustrated & Red $52 \%$ & Red $51 \%$ \\
\hline
\end{tabular}

Table 7 (continued)

\begin{tabular}{|c|c|c|}
\hline Word & Sample A & Sample B \\
\hline & Black $13 \%$ & Black $20 \%$ \\
\hline & Green $11 \%$ & Green $8 \%$ \\
\hline & Gray $4 \%$ & Gray $8 \%$ \\
\hline & Blue $6 \%$ & Blue $2 \%$ \\
\hline & Yellow 4 \% & Yellow 4 \% \\
\hline & Purple $4 \%$ & Purple $2 \%$ \\
\hline & Orange $2 \%$ & Orange $4 \%$ \\
\hline & Brown $4 \%$ & $\mathrm{TSS}=8$ \\
\hline & $\mathrm{TSS}=9$ & $\mathrm{MSS}=6$ \\
\hline & $\mathrm{MSS}=8$ & \\
\hline \multirow[t]{10}{*}{ Guilty } & Red $34 \%$ & Red $25 \%$ \\
\hline & Green $18 \%$ & Green $23 \%$ \\
\hline & Black $18 \%$ & Black $14 \%$ \\
\hline & Gray $10 \%$ & Gray $14 \%$ \\
\hline & Blue $10 \%$ & Blue $12 \%$ \\
\hline & Yellow $2 \%$ & Yellow 6 \% \\
\hline & Orange $4 \%$ & Brown 4 \% \\
\hline & White $4 \%$ & Turquoise $2 \%$ \\
\hline & $\mathrm{TSS}=8$ & $\mathrm{TSS}=8$ \\
\hline & $\mathrm{MSS}=7$ & $\mathrm{MSS}=7$ \\
\hline \multirow[t]{13}{*}{ Helpless } & Black $22 \%$ & Black $25 \%$ \\
\hline & Gray $16 \%$ & Gray $17 \%$ \\
\hline & White $18 \%$ & White $10 \%$ \\
\hline & Blue $12 \%$ & Blue $14 \%$ \\
\hline & Yellow $6 \%$ & Yellow $10 \%$ \\
\hline & Red $8 \%$ & Red $6 \%$ \\
\hline & Green $6 \%$ & Green $4 \%$ \\
\hline & Brown 4 \% & Brown $6 \%$ \\
\hline & Orange $4 \%$ & Orange $2 \%$ \\
\hline & $\operatorname{Tan} 2 \%$ & Purple $4 \%$ \\
\hline & Coral $2 \%$ & Light gray $2 \%$ \\
\hline & $\mathrm{TSS}=11$ & $\mathrm{TSS}=11$ \\
\hline & $\mathrm{MSS}=9$ & $\mathrm{MSS}=9$ \\
\hline \multirow[t]{10}{*}{ Hostile } & Red $63 \%$ & Red $56 \%$ \\
\hline & Black $21 \%$ & Black $31 \%$ \\
\hline & White $4 \%$ & White $2 \%$ \\
\hline & Gray $4 \%$ & Gray $2 \%$ \\
\hline & Yellow $2 \%$ & Blue $4 \%$ \\
\hline & Brown $2 \%$ & Green $2 \%$ \\
\hline & Dark red $2 \%$ & Purple $2 \%$ \\
\hline & Silver $2 \%$ & $\mathrm{TSS}=7$ \\
\hline & $\mathrm{TSS}=8$ & $\mathrm{MSS}=3$ \\
\hline & $\mathrm{MSS}=4$ & \\
\hline \multirow[t]{6}{*}{ Jealousy } & Green $55 \%$ & Green $61 \%$ \\
\hline & Red $23 \%$ & Red $20 \%$ \\
\hline & Blue $12 \%$ & Blue $6 \%$ \\
\hline & Yellow $2 \%$ & Yellow 6 \% \\
\hline & Black $2 \%$ & Black $2 \%$ \\
\hline & Purple $4 \%$ & Pink $2 \%$ \\
\hline
\end{tabular}


Table 7 (continued)

\begin{tabular}{|c|c|c|}
\hline Word & Sample A & Sample B \\
\hline \multirow{15}{*}{ Lonely } & Violet $2 \%$ & Brown $2 \%$ \\
\hline & $\mathrm{TSS}=7$ & $\mathrm{TSS}=7$ \\
\hline & $\mathrm{MSS}=4$ & $\mathrm{MSS}=4$ \\
\hline & Blue $44 \%$ & Blue $50 \%$ \\
\hline & Black $18 \%$ & Black $14 \%$ \\
\hline & Gray $14 \%$ & Gray $12 \%$ \\
\hline & White $6 \%$ & White $10 \%$ \\
\hline & Red $6 \%$ & Red $2 \%$ \\
\hline & Green $4 \%$ & Green $2 \%$ \\
\hline & Yellow $4 \%$ & Purple $4 \%$ \\
\hline & Light blue $2 \%$ & Pale green $2 \%$ \\
\hline & Mahogany $2 \%$ & Blue violet $2 \%$ \\
\hline & $\mathrm{TSS}=9$ & Blue gray $2 \%$ \\
\hline & $\mathrm{MSS}=7$ & $\mathrm{TSS}=10$ \\
\hline & & $\mathrm{MSS}=5$ \\
\hline \multirow[t]{7}{*}{ Mad } & Red $84 \%$ & Red $82 \%$ \\
\hline & Black $10 \%$ & Black $16 \%$ \\
\hline & Brown $2 \%$ & Blue $2 \%$ \\
\hline & Scarlett $2 \%$ & $\mathrm{TSS}=3$ \\
\hline & Dark red $2 \%$ & $\mathrm{MSS}=2$ \\
\hline & $\mathrm{TSS}=5$ & \\
\hline & $\mathrm{MSS}=2$ & \\
\hline \multirow[t]{10}{*}{ Misery } & Black $47 \%$ & Black $46 \%$ \\
\hline & $\operatorname{Red} 21 \%$ & Red $19 \%$ \\
\hline & Blue $13 \%$ & Blue $8 \%$ \\
\hline & Gray $8 \%$ & Gray $13 \%$ \\
\hline & Green $7 \%$ & Green $4 \%$ \\
\hline & White $2 \%$ & White $6 \%$ \\
\hline & Brown $2 \%$ & Purple $2 \%$ \\
\hline & $\mathrm{TSS}=7$ & Dark purple $2 \%$ \\
\hline & $\mathrm{MSS}=5$ & $\mathrm{TSS}=9$ \\
\hline & & $\mathrm{MSS}=6$ \\
\hline \multirow[t]{14}{*}{ Moody } & Blue $23 \%$ & Blue $20 \%$ \\
\hline & Gray $16 \%$ & Gray $18 \%$ \\
\hline & Red $16 \%$ & Red $10 \%$ \\
\hline & Black $12 \%$ & Black $14 \%$ \\
\hline & Green $8 \%$ & Green $10 \%$ \\
\hline & Purple $6 \%$ & Purple $10 \%$ \\
\hline & Brown $8 \%$ & Brown $8 \%$ \\
\hline & Orange $2 \%$ & Orange $2 \%$ \\
\hline & Yellow $4 \%$ & Maroon $4 \%$ \\
\hline & White $2 \%$ & Pink $2 \%$ \\
\hline & Navy $2 \%$ & Indigo $2 \%$ \\
\hline & Rainbow $2 \%$ & $\mathrm{TSS}=11$ \\
\hline & $\mathrm{TSS}=12$ & $\mathrm{MSS}=8$ \\
\hline & $\mathrm{MSS}=8$ & \\
\hline \multirow[t]{3}{*}{ Nervous } & $\operatorname{Red} 20 \%$ & $\operatorname{Red} 24 \%$ \\
\hline & Green $18 \%$ & Green $14 \%$ \\
\hline & Blue $14 \%$ & Blue $16 \%$ \\
\hline
\end{tabular}

Table 7 (continued)

\begin{tabular}{|c|c|c|}
\hline Word & Sample A & Sample B \\
\hline & Yellow 14 \% & Yellow 14 \% \\
\hline & Gray $10 \%$ & Gray $6 \%$ \\
\hline & Orange $8 \%$ & Orange $8 \%$ \\
\hline & Black $6 \%$ & Black $4 \%$ \\
\hline & Brown $4 \%$ & Brown $6 \%$ \\
\hline & White $2 \%$ & White $6 \%$ \\
\hline & Light green $2 \%$ & Silver $2 \%$ \\
\hline & Fuchsia $2 \%$ & $\mathrm{TSS}=10$ \\
\hline & $\mathrm{TSS}=11$ & $\mathrm{MSS}=9$ \\
\hline & $\mathrm{MSS}=8$ & \\
\hline \multirow[t]{10}{*}{ Panic } & Red $69 \%$ & Red $73 \%$ \\
\hline & Black $6 \%$ & Black $10 \%$ \\
\hline & Blue $10 \%$ & Blue $6 \%$ \\
\hline & Yellow 4 \% & Yellow 4 \% \\
\hline & White $4 \%$ & Green $2 \%$ \\
\hline & Green $2 \%$ & Purple $2 \%$ \\
\hline & Brown $2 \%$ & Orange $2 \%$ \\
\hline & Beige $2 \%$ & Indian red $2 \%$ \\
\hline & $\mathrm{TSS}=8$ & $\mathrm{TSS}=8$ \\
\hline & $\mathrm{MSS}=5$ & $\mathrm{MSS}=4$ \\
\hline \multirow[t]{6}{*}{ Rage } & Red $86 \%$ & Red $94 \%$ \\
\hline & Black $10 \%$ & Black $6 \%$ \\
\hline & Pink $2 \%$ & $\mathrm{TSS}=2$ \\
\hline & Green $2 \%$ & $\mathrm{MSS}=2$ \\
\hline & $\mathrm{TSS}=4$ & \\
\hline & $\mathrm{MSS}=2$ & \\
\hline \multirow[t]{11}{*}{ Regretful } & Red $29 \%$ & Red $28 \%$ \\
\hline & Black $31 \%$ & Black $24 \%$ \\
\hline & Blue $12 \%$ & Blue $20 \%$ \\
\hline & Green $10 \%$ & Green $6 \%$ \\
\hline & Gray $8 \%$ & Gray $6 \%$ \\
\hline & Yellow 4 \% & Yellow 7 \% \\
\hline & Purple $2 \%$ & Purple $4 \%$ \\
\hline & Orange $2 \%$ & White $2 \%$ \\
\hline & Brown $2 \%$ & Pink $2 \%$ \\
\hline & $\mathrm{TSS}=9$ & $\mathrm{TSS}=9$ \\
\hline & $\mathrm{MSS}=6$ & $\mathrm{MSS}=7$ \\
\hline \multirow[t]{11}{*}{ Sad } & Blue $60 \%$ & Blue $60 \%$ \\
\hline & Black $18 \%$ & Black $19 \%$ \\
\hline & Red $8 \%$ & Red $6 \%$ \\
\hline & Gray $2 \%$ & Gray $6 \%$ \\
\hline & Yellow 4 \% & Yellow 4 \% \\
\hline & Green $4 \%$ & Green $2 \%$ \\
\hline & Purple $2 \%$ & Pink $2 \%$ \\
\hline & Brown $2 \%$ & Midnight blue \\
\hline & $\mathrm{TSS}=8$ & $2 \%$ \\
\hline & $\mathrm{MSS}=5$ & $\mathrm{TSS}=8$ \\
\hline & & $\mathrm{MSS}=5$ \\
\hline Scared & Red $44 \%$ & Red $33 \%$ \\
\hline
\end{tabular}


Table 7 (continued)

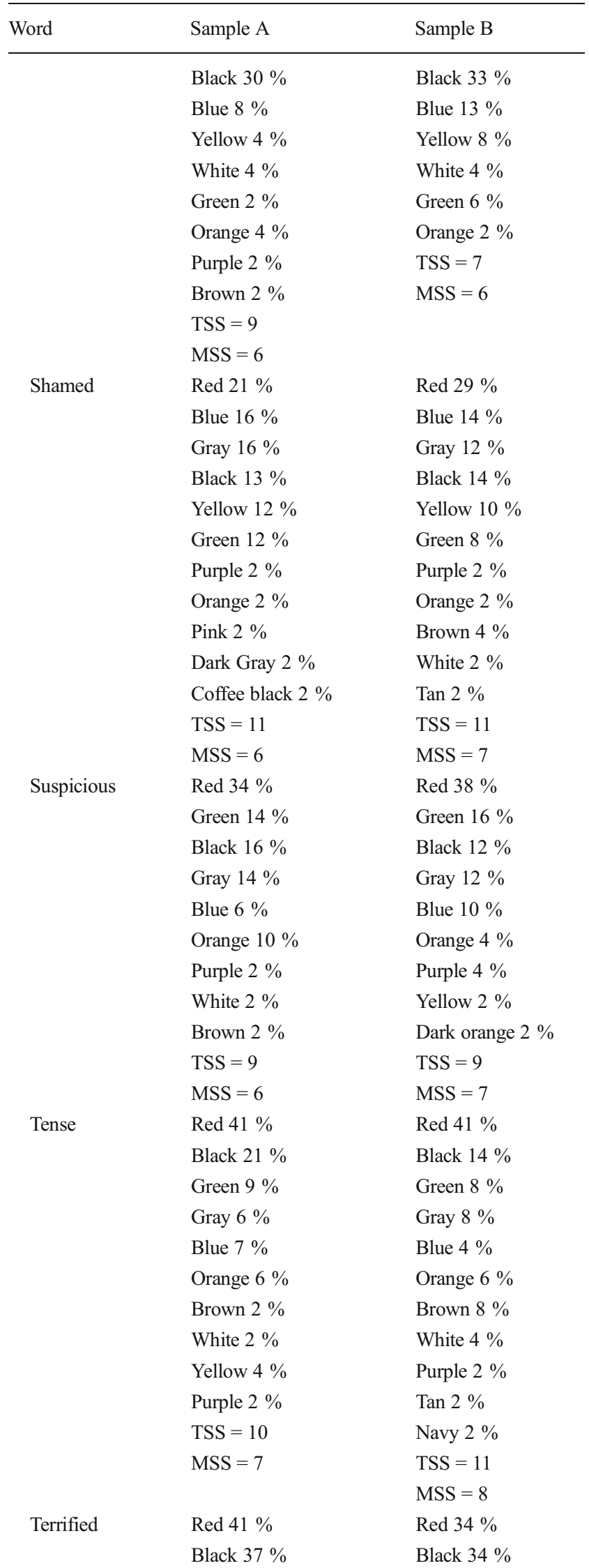

Table 7 (continued)

\begin{tabular}{|c|c|c|}
\hline Word & Sample A & Sample B \\
\hline \multirow{21}{*}{ Troubled } & Blue $10 \%$ & Blue $8 \%$ \\
\hline & Gray 4 \% & Gray $8 \%$ \\
\hline & Green $6 \%$ & Green $6 \%$ \\
\hline & White $2 \%$ & White $4 \%$ \\
\hline & $\mathrm{TSS}=6$ & Yellow $2 \%$ \\
\hline & \multirow[t]{4}{*}{$\mathrm{MSS}=5$} & Orange $2 \%$ \\
\hline & & Deep purple $2 \%$ \\
\hline & & $\mathrm{TSS}=9$ \\
\hline & & $\mathrm{MSS}=6$ \\
\hline & Red $33 \%$ & Red $30 \%$ \\
\hline & Blue $16 \%$ & Blue $18 \%$ \\
\hline & Gray $12 \%$ & Gray $14 \%$ \\
\hline & Green $14 \%$ & Green $12 \%$ \\
\hline & Black $12 \%$ & Black $14 \%$ \\
\hline & Brown $4 \%$ & Brown $6 \%$ \\
\hline & Orange $4 \%$ & Yellow $2 \%$ \\
\hline & White $2 \%$ & Pink $2 \%$ \\
\hline & Purple $2 \%$ & Dark gray $2 \%$ \\
\hline & Silver $2 \%$ & $\mathrm{TSS}=9$ \\
\hline & $\mathrm{TSS}=10$ & $\mathrm{MSS}=6$ \\
\hline & $\mathrm{MSS}=7$ & \\
\hline \multirow[t]{11}{*}{ Unhappy } & Blue $38 \%$ & Blue $44 \%$ \\
\hline & Black $20 \%$ & Black $18 \%$ \\
\hline & Gray $18 \%$ & Gray $12 \%$ \\
\hline & Red $10 \%$ & Red $12 \%$ \\
\hline & Yellow $2 \%$ & Yellow $6 \%$ \\
\hline & Brown 4 \% & Brown 4 \% \\
\hline & Orange $2 \%$ & Orange $2 \%$ \\
\hline & Green $4 \%$ & White $2 \%$ \\
\hline & Purple $2 \%$ & $\mathrm{TSS}=8$ \\
\hline & $\mathrm{TSS}=9$ & $\mathrm{MSS}=6$ \\
\hline & $\mathrm{MSS}=6$ & \\
\hline \multirow[t]{11}{*}{ Upset } & Blue $41 \%$ & Blue $41 \%$ \\
\hline & $\operatorname{Red} 29 \%$ & $\operatorname{Red} 25 \%$ \\
\hline & Black $12 \%$ & Black $16 \%$ \\
\hline & Gray $10 \%$ & Gray $6 \%$ \\
\hline & Brown $2 \%$ & Brown $4 \%$ \\
\hline & Purple $2 \%$ & Yellow $2 \%$ \\
\hline & Orange $2 \%$ & Green $2 \%$ \\
\hline & Navy $2 \%$ & Dark red $2 \%$ \\
\hline & $\mathrm{TSS}=8$ & Peach $2 \%$ \\
\hline & $\mathrm{MSS}=4$ & $\mathrm{TSS}=9$ \\
\hline & & $\mathrm{MSS}=5$ \\
\hline \multicolumn{3}{|c|}{ Negative Emotion-Laden } \\
\hline \multirow[t]{5}{*}{ Abduction } & Black $45 \%$ & Black 43 \% \\
\hline & $\operatorname{Red} 21 \%$ & $\operatorname{Red} 25 \%$ \\
\hline & Green $8 \%$ & Green $12 \%$ \\
\hline & Yellow 6 \% & Yellow $6 \%$ \\
\hline & Blue $6 \%$ & Blue $4 \%$ \\
\hline
\end{tabular}


Table 7 (continued)

\begin{tabular}{|c|c|c|}
\hline Word & Sample A & Sample B \\
\hline & Gray $4 \%$ & Gray $2 \%$ \\
\hline & Brown $2 \%$ & Brown $4 \%$ \\
\hline & Orange $2 \%$ & Orange $4 \%$ \\
\hline & Pink $2 \%$ & $\mathrm{TSS}=8$ \\
\hline & Silver $2 \%$ & $\mathrm{MSS}=7$ \\
\hline & Dark red $2 \%$ & \\
\hline & $\mathrm{TSS}=11$ & \\
\hline & $\mathrm{MSS}=6$ & \\
\hline \multirow[t]{11}{*}{ Abortion } & Red $54 \%$ & Red $46 \%$ \\
\hline & Black $20 \%$ & Black $28 \%$ \\
\hline & Blue $8 \%$ & Blue $4 \%$ \\
\hline & White $4 \%$ & White $6 \%$ \\
\hline & Gray $2 \%$ & Gray $6 \%$ \\
\hline & Yellow $4 \%$ & Yellow 4 \% \\
\hline & Pink $4 \%$ & Pink $2 \%$ \\
\hline & Dark red $2 \%$ & Green $4 \%$ \\
\hline & Brown $2 \%$ & $\mathrm{TSS}=8$ \\
\hline & $\mathrm{TSS}=9$ & $\mathrm{MSS}=7$ \\
\hline & $\mathrm{MSS}=6$ & \\
\hline \multirow[t]{8}{*}{ Abuse } & Red $47 \%$ & $\operatorname{Red} 49 \%$ \\
\hline & Black $37 \%$ & Black $41 \%$ \\
\hline & Blue $6 \%$ & Blue $2 \%$ \\
\hline & Gray $6 \%$ & Gray $2 \%$ \\
\hline & White $2 \%$ & Green $4 \%$ \\
\hline & Purple $2 \%$ & Brown $2 \%$ \\
\hline & $\mathrm{TSS}=6$ & $\mathrm{TSS}=6$ \\
\hline & $\mathrm{MSS}=4$ & $\mathrm{MSS}=3$ \\
\hline \multirow[t]{4}{*}{ Bees } & Yellow $100 \%$ & Yellow $98 \%$ \\
\hline & $\mathrm{TSS}=1$ & Black $2 \%$ \\
\hline & $\mathrm{MSS}=1$ & $\mathrm{TSS}=2$ \\
\hline & & $\mathrm{MSS}=1$ \\
\hline \multirow[t]{6}{*}{ Bloody } & Red $94 \%$ & Red $98 \%$ \\
\hline & Wine red $2 \%$ & Crimson $2 \%$ \\
\hline & Black $2 \%$ & $\mathrm{TSS}=2$ \\
\hline & Blue $2 \%$ & $\mathrm{MSS}=1$ \\
\hline & $\mathrm{TSS}=4$ & \\
\hline & $\mathrm{MSS}=1$ & \\
\hline \multirow[t]{7}{*}{ Burial } & Black $49 \%$ & Black $44 \%$ \\
\hline & Brown $37 \%$ & Brown $46 \%$ \\
\hline & Gray $10 \%$ & Gray $6 \%$ \\
\hline & $\operatorname{Red} 2 \%$ & Red $2 \%$ \\
\hline & Dark brown $2 \%$ & White $2 \%$ \\
\hline & $\mathrm{TSS}=5$ & $\mathrm{TSS}=5$ \\
\hline & $\mathrm{MSS}=3$ & $\mathrm{MSS}=3$ \\
\hline \multirow[t]{5}{*}{ Cancer } & Black $32 \%$ & Black $34 \%$ \\
\hline & Pink $18 \%$ & Pink $23 \%$ \\
\hline & $\operatorname{Red} 22 \%$ & Red $17 \%$ \\
\hline & White $4 \%$ & White $9 \%$ \\
\hline & Yellow $6 \%$ & Yellow 4 \% \\
\hline
\end{tabular}

Table 7 (continued)

\begin{tabular}{|c|c|c|}
\hline Word & Sample A & Sample B \\
\hline & Brown $4 \%$ & Brown $5 \%$ \\
\hline & Blue $6 \%$ & Blue $2 \%$ \\
\hline & Gray $2 \%$ & Gray $4 \%$ \\
\hline & Orange $4 \%$ & Tan $2 \%$ \\
\hline & Green $2 \%$ & $\mathrm{TSS}=9$ \\
\hline & $\mathrm{TSS}=10$ & $\mathrm{MSS}=7$ \\
\hline & $\mathrm{MSS}=8$ & \\
\hline \multirow[t]{9}{*}{ Cemetery } & Black $60 \%$ & Black $49 \%$ \\
\hline & Gray $30 \%$ & Gray $35 \%$ \\
\hline & White $4 \%$ & White $8 \%$ \\
\hline & Brown $2 \%$ & Brown $2 \%$ \\
\hline & Green $4 \%$ & Forrest green $2 \%$ \\
\hline & $\mathrm{TSS}=5$ & Silver $2 \%$ \\
\hline & $\mathrm{MSS}=4$ & Red $2 \%$ \\
\hline & & $\mathrm{TSS}=7$ \\
\hline & & $\mathrm{MSS}=3$ \\
\hline \multirow[t]{8}{*}{ Coffin } & Brown $56 \%$ & Brown $50 \%$ \\
\hline & Black $36 \%$ & Black $42 \%$ \\
\hline & Orange $2 \%$ & Gray $4 \%$ \\
\hline & Tan $2 \%$ & Silver $2 \%$ \\
\hline & Magenta $2 \%$ & Mahogany $2 \%$ \\
\hline & Cream $2 \%$ & $\mathrm{TSS}=5$ \\
\hline & $\mathrm{TSS}=6$ & $\mathrm{MSS}=3$ \\
\hline & $\mathrm{MSS}=2$ & \\
\hline \multirow[t]{11}{*}{ Corpse } & Black $53 \%$ & Black $44 \%$ \\
\hline & White $17 \%$ & White $19 \%$ \\
\hline & Gray $12 \%$ & Gray $15 \%$ \\
\hline & Brown $4 \%$ & Brown $8 \%$ \\
\hline & Blue $6 \%$ & Blue $4 \%$ \\
\hline & Red $6 \%$ & Red $4 \%$ \\
\hline & Yellow $2 \%$ & Pink $2 \%$ \\
\hline & $\mathrm{TSS}=7$ & Red $2 \%$ \\
\hline & $\mathrm{MSS}=6$ & White $2 \%$ \\
\hline & & $\mathrm{TSS}=9$ \\
\hline & & $\mathrm{MSS}=6$ \\
\hline \multirow[t]{11}{*}{ Crash } & $\operatorname{Red} 51 \%$ & Red $54 \%$ \\
\hline & Black $21 \%$ & Black $30 \%$ \\
\hline & Gray $10 \%$ & Gray $4 \%$ \\
\hline & Blue $4 \%$ & Blue $6 \%$ \\
\hline & Brown $2 \%$ & Brown $4 \%$ \\
\hline & Yellow $2 \%$ & Yellow $2 \%$ \\
\hline & Green $6 \%$ & $\mathrm{TSS}=6$ \\
\hline & Purple $2 \%$ & $\mathrm{MSS}=5$ \\
\hline & Orange $2 \%$ & \\
\hline & $\mathrm{TSS}=9$ & \\
\hline & $\mathrm{MSS}=5$ & \\
\hline \multirow[t]{3}{*}{ Criminal } & Black $54 \%$ & Black $58 \%$ \\
\hline & Orange $14 \%$ & Orange $10 \%$ \\
\hline & Red $8 \%$ & Red $14 \%$ \\
\hline
\end{tabular}


Table 7 (continued)

\begin{tabular}{|c|c|c|}
\hline Word & Sample A & Sample B \\
\hline \multirow{17}{*}{ Crisis } & Gray $10 \%$ & Gray $8 \%$ \\
\hline & White $6 \%$ & White $2 \%$ \\
\hline & Brown $4 \%$ & Brown $4 \%$ \\
\hline & Blue $2 \%$ & Green $4 \%$ \\
\hline & Pink $2 \%$ & $\mathrm{TSS}=7$ \\
\hline & $\mathrm{TSS}=8$ & $\mathrm{MSS}=6$ \\
\hline & $\mathrm{MSS}=6$ & \\
\hline & Red $55 \%$ & Red $62 \%$ \\
\hline & Black $21 \%$ & Black $16 \%$ \\
\hline & Blue $4 \%$ & Blue $10 \%$ \\
\hline & Gray $10 \%$ & Gray $4 \%$ \\
\hline & Orange $2 \%$ & Orange $2 \%$ \\
\hline & Green $4 \%$ & Yellow 4 \% \\
\hline & Brown $2 \%$ & Beige $2 \%$ \\
\hline & Dark red $2 \%$ & $\mathrm{TSS}=7$ \\
\hline & $\mathrm{TSS}=8$ & $\mathrm{MSS}=5$ \\
\hline & $\mathrm{MSS}=5$ & \\
\hline \multirow[t]{7}{*}{ Danger } & Red $86 \%$ & Red $78 \%$ \\
\hline & Black $8 \%$ & Black $10 \%$ \\
\hline & Orange $2 \%$ & Orange $6 \%$ \\
\hline & Yellow $4 \%$ & Yellow $2 \%$ \\
\hline & $\mathrm{TSS}=4$ & Dark red $2 \%$ \\
\hline & $\mathrm{MSS}=3$ & $\mathrm{TSS}=5$ \\
\hline & & $\mathrm{MSS}=3$ \\
\hline \multirow[t]{9}{*}{ Dead } & Black $75 \%$ & Black $77 \%$ \\
\hline & Red $10 \%$ & Red $13 \%$ \\
\hline & White $4 \%$ & White $2 \%$ \\
\hline & Gray $2 \%$ & Gray $2 \%$ \\
\hline & Brown $2 \%$ & Brown $2 \%$ \\
\hline & Blue $4 \%$ & Green $4 \%$ \\
\hline & Purple $2 \%$ & $\mathrm{TSS}=6$ \\
\hline & $\mathrm{TSS}=7$ & $\mathrm{MSS}=3$ \\
\hline & $\mathrm{MSS}=4$ & \\
\hline \multirow[t]{6}{*}{ Death } & Black $88 \%$ & Black 84 \% \\
\hline & Red $10 \%$ & Red $12 \%$ \\
\hline & Orange $2 \%$ & White $2 \%$ \\
\hline & $\mathrm{TSS}=3$ & Gray $2 \%$ \\
\hline & $\mathrm{MSS}=2$ & $\mathrm{TSS}=4$ \\
\hline & & $\mathrm{MSS}=2$ \\
\hline \multirow[t]{7}{*}{ Demon } & Red $63 \%$ & Red $71 \%$ \\
\hline & Black $31 \%$ & Black $25 \%$ \\
\hline & Blue $2 \%$ & Gray $2 \%$ \\
\hline & Purple $2 \%$ & Green $2 \%$ \\
\hline & Brown $2 \%$ & $\mathrm{TSS}=4$ \\
\hline & $\mathrm{TSS}=5$ & $\mathrm{MSS}=2$ \\
\hline & $\mathrm{MSS}=2$ & \\
\hline \multirow[t]{3}{*}{ Disaster } & Black $38 \%$ & Black $42 \%$ \\
\hline & Red $34 \%$ & Red $28 \%$ \\
\hline & Gray $14 \%$ & Gray $8 \%$ \\
\hline
\end{tabular}

Table 7 (continued)

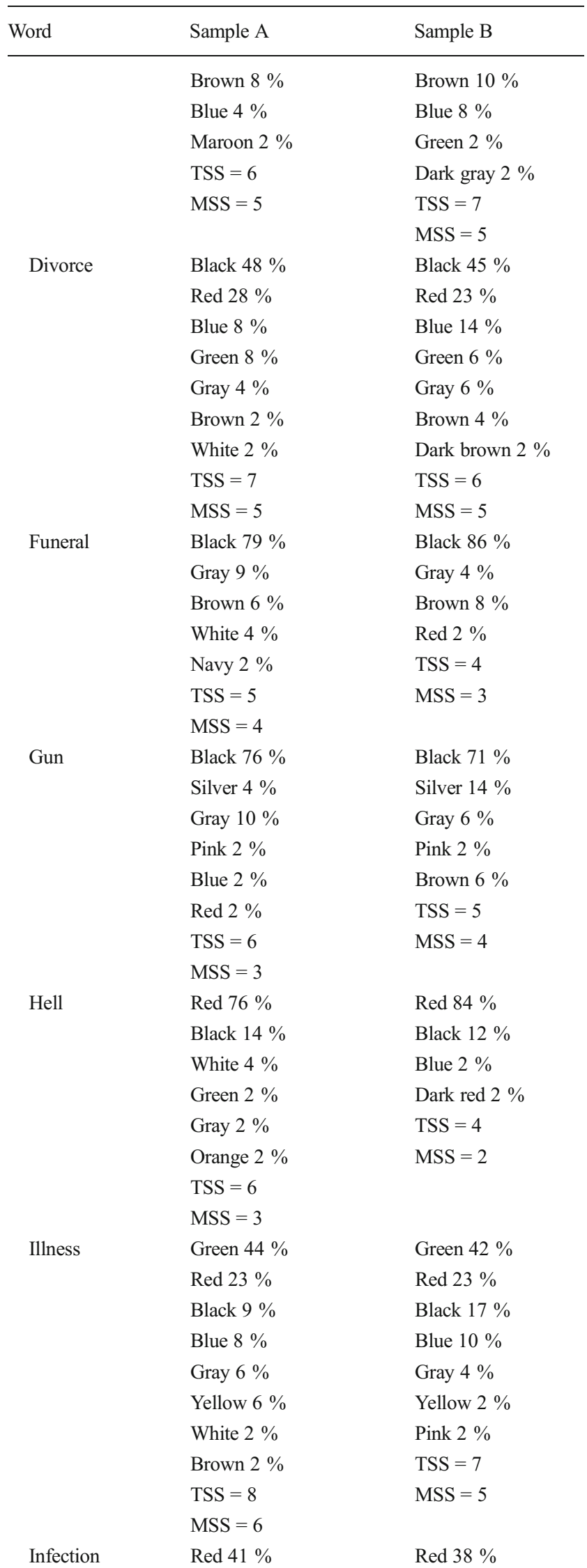


Table 7 (continued)

\begin{tabular}{|c|c|c|}
\hline Word & Sample A & Sample B \\
\hline & Green $25 \%$ & Green $22 \%$ \\
\hline & Black $10 \%$ & Black $16 \%$ \\
\hline & Brown $10 \%$ & Brown $6 \%$ \\
\hline & White $4 \%$ & White $6 \%$ \\
\hline & Yellow 4 \% & Yellow 4 \% \\
\hline & Pink $2 \%$ & Pink 4 \% \\
\hline & Gray $4 \%$ & Blue $2 \%$ \\
\hline & $\mathrm{TSS}=8$ & Dark magenta $2 \%$ \\
\hline & $\mathrm{MSS}=7$ & $\mathrm{TSS}=9$ \\
\hline & & $\mathrm{MSS}=7$ \\
\hline \multirow[t]{12}{*}{ Injury } & Red $57 \%$ & Red $66 \%$ \\
\hline & Black $15 \%$ & Black $14 \%$ \\
\hline & Blue $6 \%$ & Blue $4 \%$ \\
\hline & Brown $8 \%$ & Brown $2 \%$ \\
\hline & White $4 \%$ & White $2 \%$ \\
\hline & Purple $2 \%$ & Purple $2 \%$ \\
\hline & Orange $2 \%$ & Orange $2 \%$ \\
\hline & Pink $6 \%$ & Gray $4 \%$ \\
\hline & $\mathrm{TSS}=8$ & Green $2 \%$ \\
\hline & $\mathrm{MSS}=6$ & Dark red $2 \%$ \\
\hline & & $\mathrm{TSS}=10$ \\
\hline & & $\mathrm{MSS}=4$ \\
\hline \multirow[t]{12}{*}{ Insult } & Red $33 \%$ & Red $25 \%$ \\
\hline & Black $25 \%$ & Black $27 \%$ \\
\hline & Green $12 \%$ & Green $16 \%$ \\
\hline & Gray $4 \%$ & Gray $12 \%$ \\
\hline & Blue $10 \%$ & Blue $4 \%$ \\
\hline & Brown $6 \%$ & Brown $8 \%$ \\
\hline & Purple $4 \%$ & Purple $2 \%$ \\
\hline & Silver $2 \%$ & White $4 \%$ \\
\hline & Tan $2 \%$ & Yellow $2 \%$ \\
\hline & Magenta $2 \%$ & $\mathrm{TSS}=9$ \\
\hline & $\mathrm{TSS}=10$ & $\mathrm{MSS}=7$ \\
\hline & $\mathrm{MSS}=7$ & \\
\hline \multirow[t]{8}{*}{ Jail } & Gray $41 \%$ & Gray $41 \%$ \\
\hline & Black $33 \%$ & Black $29 \%$ \\
\hline & Orange $10 \%$ & Orange $12 \%$ \\
\hline & Silver $8 \%$ & Silver $14 \%$ \\
\hline & Brown $6 \%$ & Red $2 \%$ \\
\hline & Blue $2 \%$ & Black and white $2 \%$ \\
\hline & $\mathrm{TSS}=6$ & $\mathrm{TSS}=6$ \\
\hline & $\mathrm{MSS}=5$ & $\mathrm{MSS}=4$ \\
\hline \multirow[t]{7}{*}{ Killer } & Red $56 \%$ & Red $52 \%$ \\
\hline & Black $38 \%$ & Black $34 \%$ \\
\hline & White $2 \%$ & White $4 \%$ \\
\hline & Scarlett $2 \%$ & Gray $4 \%$ \\
\hline & Brown $2 \%$ & Blue $2 \%$ \\
\hline & $\mathrm{TSS}=5$ & Dark red $2 \%$ \\
\hline & $\mathrm{MSS}=2$ & Charcoal $2 \%$ \\
\hline
\end{tabular}

Table 7 (continued)

\begin{tabular}{|c|c|c|}
\hline Word & Sample A & Sample B \\
\hline & & $\mathrm{TSS}=7$ \\
\hline & & $\mathrm{MSS}=4$ \\
\hline \multirow[t]{11}{*}{ Lie } & Black $21 \%$ & Black $24 \%$ \\
\hline & $\operatorname{Red} 22 \%$ & $\operatorname{Red} 22 \%$ \\
\hline & White $22 \%$ & White $27 \%$ \\
\hline & Green $12 \%$ & Green $13 \%$ \\
\hline & Blue $10 \%$ & Blue $5 \%$ \\
\hline & Gray $2 \%$ & Gray $3 \%$ \\
\hline & Orange $2 \%$ & Yellow $2 \%$ \\
\hline & Brown $2 \%$ & Purple $2 \%$ \\
\hline & $\mathrm{TSS}=8$ & Maroon $2 \%$ \\
\hline & $\mathrm{MSS}=5$ & $\mathrm{TSS}=9$ \\
\hline & & $\mathrm{MSS}=6$ \\
\hline \multirow[t]{8}{*}{ Massacre } & Red $76 \%$ & Red $82 \%$ \\
\hline & Black $16 \%$ & Black $10 \%$ \\
\hline & Blue $2 \%$ & Blue $4 \%$ \\
\hline & White $2 \%$ & Green $2 \%$ \\
\hline & Gold $2 \%$ & Brown $2 \%$ \\
\hline & Dark red $2 \%$ & $\mathrm{TSS}=5$ \\
\hline & $\mathrm{TSS}=6$ & $\mathrm{MSS}=3$ \\
\hline & $\mathrm{MSS}=2$ & \\
\hline \multirow[t]{9}{*}{ Morbid } & Black $72 \%$ & Black $64 \%$ \\
\hline & Red $12 \%$ & Red $10 \%$ \\
\hline & Gray $6 \%$ & Gray $12 \%$ \\
\hline & White $2 \%$ & White $6 \%$ \\
\hline & Brown $4 \%$ & Brown $2 \%$ \\
\hline & Green $2 \%$ & Green $2 \%$ \\
\hline & Yellow $2 \%$ & Blue $4 \%$ \\
\hline & $\mathrm{TSS}=7$ & $\mathrm{TSS}=7$ \\
\hline & $\mathrm{MSS}=4$ & $\mathrm{MSS}=5$ \\
\hline \multirow[t]{8}{*}{ Nightmare } & Black $70 \%$ & Black $78 \%$ \\
\hline & Red $14 \%$ & Red $10 \%$ \\
\hline & Blue $6 \%$ & Blue $4 \%$ \\
\hline & Gray $4 \%$ & Gray $2 \%$ \\
\hline & Green $6 \%$ & Purple $4 \%$ \\
\hline & $\mathrm{TSS}=5$ & Brown $2 \%$ \\
\hline & $\mathrm{MSS}=5$ & $\mathrm{TSS}=6$ \\
\hline & & $\mathrm{MSS}=4$ \\
\hline \multirow[t]{11}{*}{ Pain } & Red $74 \%$ & Red $68 \%$ \\
\hline & Black $6 \%$ & Black $10 \%$ \\
\hline & Blue $8 \%$ & Blue $4 \%$ \\
\hline & Yellow $2 \%$ & Yellow $4 \%$ \\
\hline & White $4 \%$ & Gray $4 \%$ \\
\hline & Cream $2 \%$ & Green $4 \%$ \\
\hline & Orange $2 \%$ & Purple $2 \%$ \\
\hline & Dark red $2 \%$ & Brown $2 \%$ \\
\hline & $\mathrm{TSS}=8$ & Flesh $2 \%$ \\
\hline & $\mathrm{MSS}=4$ & $\mathrm{TSS}=9$ \\
\hline & & $\mathrm{MSS}=6$ \\
\hline
\end{tabular}


Table 7 (continued)

\begin{tabular}{|c|c|c|}
\hline Word & Sample A & Sample B \\
\hline \multirow[t]{10}{*}{ Poison } & Black $32 \%$ & Black $37 \%$ \\
\hline & Green $27 \%$ & Green $27 \%$ \\
\hline & $\operatorname{Red} 29 \%$ & $\operatorname{Red} 22 \%$ \\
\hline & Yellow $2 \%$ & Yellow $4 \%$ \\
\hline & Blue $2 \%$ & Blue $2 \%$ \\
\hline & Clear $2 \%$ & Clear $2 \%$ \\
\hline & White $4 \%$ & Purple $4 \%$ \\
\hline & Lime green $2 \%$ & Gray $2 \%$ \\
\hline & $\mathrm{TSS}=8$ & $\mathrm{TSS}=8$ \\
\hline & $\mathrm{MSS}=4$ & $\mathrm{MSS}=5$ \\
\hline \multirow[t]{9}{*}{ Pollute } & Gray $36 \%$ & Gray $41 \%$ \\
\hline & Black $38 \%$ & Black $35 \%$ \\
\hline & Green $8 \%$ & Green $12 \%$ \\
\hline & Brown $12 \%$ & Brown $8 \%$ \\
\hline & White $2 \%$ & Blue $2 \%$ \\
\hline & Yellow $2 \%$ & Red $2 \%$ \\
\hline & Dark gray $2 \%$ & $\mathrm{TSS}=6$ \\
\hline & $\mathrm{TSS}=7$ & $\mathrm{MSS}=4$ \\
\hline & $\mathrm{MSS}=4$ & \\
\hline \multirow[t]{12}{*}{ Poverty } & Black $31 \%$ & Black $35 \%$ \\
\hline & Brown $25 \%$ & Brown $23 \%$ \\
\hline & Gray $16 \%$ & Gray $18 \%$ \\
\hline & Blue $10 \%$ & Blue $6 \%$ \\
\hline & Green $8 \%$ & Green $4 \%$ \\
\hline & Red $2 \%$ & Red $6 \%$ \\
\hline & Orange $2 \%$ & Orange $4 \%$ \\
\hline & Pink $2 \%$ & Yellow $2 \%$ \\
\hline & Silver $2 \%$ & Gold $2 \%$ \\
\hline & Crimson $2 \%$ & $\mathrm{TSS}=9$ \\
\hline & $\mathrm{TSS}=10$ & $\mathrm{MSS}=7$ \\
\hline & $\mathrm{MSS}=5$ & \\
\hline \multirow[t]{11}{*}{ Punishment } & Red $52 \%$ & Red $46 \%$ \\
\hline & Black $34 \%$ & Black $30 \%$ \\
\hline & Blue $4 \%$ & Blue $6 \%$ \\
\hline & Gray $2 \%$ & Gray $4 \%$ \\
\hline & Purple $2 \%$ & Purple $2 \%$ \\
\hline & Green $4 \%$ & White $4 \%$ \\
\hline & Silver $2 \%$ & Brown $4 \%$ \\
\hline & $\mathrm{TSS}=7$ & Yellow $2 \%$ \\
\hline & $\mathrm{MSS}=4$ & Dark red $2 \%$ \\
\hline & & $\mathrm{TSS}=9$ \\
\hline & & $\mathrm{MSS}=6$ \\
\hline \multirow[t]{7}{*}{ Rape } & Red $57 \%$ & Red $57 \%$ \\
\hline & Black $37 \%$ & Black $37 \%$ \\
\hline & Gray $4 \%$ & White $2 \%$ \\
\hline & Blue $2 \%$ & Purple $2 \%$ \\
\hline & $\mathrm{TSS}=4$ & Brown $2 \%$ \\
\hline & $\mathrm{MSS}=3$ & $\mathrm{TSS}=5$ \\
\hline & & $\mathrm{MSS}=2$ \\
\hline
\end{tabular}

Table 7 (continued)

\begin{tabular}{|c|c|c|}
\hline Word & Sample A & Sample B \\
\hline \multirow[t]{7}{*}{ Robber } & Black $84 \%$ & Black $82 \%$ \\
\hline & Red $8 \%$ & Red $12 \%$ \\
\hline & Green $4 \%$ & White $2 \%$ \\
\hline & Blue $2 \%$ & Gray $2 \%$ \\
\hline & Brown $2 \%$ & Beige $2 \%$ \\
\hline & $\mathrm{TSS}=5$ & $\mathrm{TSS}=5$ \\
\hline & $\mathrm{MSS}=3$ & $\mathrm{MSS}=2$ \\
\hline \multirow[t]{12}{*}{ Sick } & Green $59 \%$ & Green $60 \%$ \\
\hline & Blue $6 \%$ & Blue $10 \%$ \\
\hline & Red $9 \%$ & $\operatorname{Red} 4 \%$ \\
\hline & Brown $6 \%$ & Brown $6 \%$ \\
\hline & Gray $6 \%$ & Gray $4 \%$ \\
\hline & Black $2 \%$ & Black $6 \%$ \\
\hline & White $4 \%$ & White $2 \%$ \\
\hline & Orange $4 \%$ & Yellow $6 \%$ \\
\hline & Tan $2 \%$ & Orange $2 \%$ \\
\hline & Pale green $2 \%$ & $\mathrm{TSS}=9$ \\
\hline & $\mathrm{TSS}=10$ & $\mathrm{MSS}=7$ \\
\hline & $\mathrm{MSS}=7$ & \\
\hline \multirow[t]{11}{*}{ Sickness } & Green $44 \%$ & Green $48 \%$ \\
\hline & Red $16 \%$ & Red $19 \%$ \\
\hline & Black $14 \%$ & Black $9 \%$ \\
\hline & Blue $10 \%$ & Blue $6 \%$ \\
\hline & Yellow $4 \%$ & Yellow $8 \%$ \\
\hline & Brown $4 \%$ & Brown $6 \%$ \\
\hline & Gray $4 \%$ & White $2 \%$ \\
\hline & Beige $2 \%$ & Dark blue $2 \%$ \\
\hline & Pale green $2 \%$ & $\mathrm{TSS}=8$ \\
\hline & $\mathrm{TSS}=9$ & $\mathrm{MSS}=6$ \\
\hline & $\mathrm{MSS}=7$ & \\
\hline \multirow[t]{9}{*}{ Slave } & Black $70 \%$ & Black $66 \%$ \\
\hline & Brown $10 \%$ & Brown $14 \%$ \\
\hline & Red $8 \%$ & Red $8 \%$ \\
\hline & Gray $6 \%$ & Gray $2 \%$ \\
\hline & Green $2 \%$ & Green $4 \%$ \\
\hline & Yellow $2 \%$ & Yellow $2 \%$ \\
\hline & Blue $2 \%$ & White $4 \%$ \\
\hline & $\mathrm{TSS}=7$ & $\mathrm{TSS}=7$ \\
\hline & $\mathrm{MSS}=4$ & $\mathrm{MSS}=5$ \\
\hline \multirow[t]{8}{*}{ Suicide } & $\operatorname{Red} 47 \%$ & $\operatorname{Red} 44 \%$ \\
\hline & Black $43 \%$ & Black $42 \%$ \\
\hline & Blue $4 \%$ & Blue $6 \%$ \\
\hline & White $2 \%$ & White $2 \%$ \\
\hline & Green $2 \%$ & Gray $6 \%$ \\
\hline & Orange $2 \%$ & $\mathrm{TSS}=5$ \\
\hline & $\mathrm{TSS}=6$ & $\mathrm{MSS}=4$ \\
\hline & $\mathrm{MSS}=3$ & \\
\hline \multirow[t]{2}{*}{ Tomb } & Black $37 \%$ & Black $42 \%$ \\
\hline & Gray $27 \%$ & Gray $29 \%$ \\
\hline
\end{tabular}


Table 7 (continued)

\begin{tabular}{|c|c|c|}
\hline Word & Sample A & Sample B \\
\hline & Brown $20 \%$ & Brown $13 \%$ \\
\hline & Yellow 4 \% & Yellow 4 \% \\
\hline & Red $2 \%$ & Red $4 \%$ \\
\hline & Gold $4 \%$ & Beige $2 \%$ \\
\hline & Beige $2 \%$ & Blue $2 \%$ \\
\hline & Green $2 \%$ & $\operatorname{Tan} 2 \%$ \\
\hline & Sandstone $2 \%$ & Burlywood $2 \%$ \\
\hline & $\mathrm{TSS}=9$ & $\mathrm{TSS}=9$ \\
\hline & $\mathrm{MSS}=5$ & $\mathrm{MSS}=5$ \\
\hline \multirow[t]{10}{*}{ Toothache } & White $45 \%$ & White $47 \%$ \\
\hline & Red $34 \%$ & Red $31 \%$ \\
\hline & Black $6 \%$ & Black $10 \%$ \\
\hline & Yellow $6 \%$ & Yellow $2 \%$ \\
\hline & Gray $3 \%$ & Gray $2 \%$ \\
\hline & Green $2 \%$ & Blue $4 \%$ \\
\hline & Brown $2 \%$ & Pale yellow $2 \%$ \\
\hline & Dark red $2 \%$ & Purple $2 \%$ \\
\hline & $\mathrm{TSS}=8$ & $\mathrm{TSS}=8$ \\
\hline & $\mathrm{MSS}=5$ & $\mathrm{MSS}=4$ \\
\hline \multirow[t]{11}{*}{ Tornado } & Gray $47 \%$ & Gray $51 \%$ \\
\hline & Black $17 \%$ & Black $19 \%$ \\
\hline & Blue $14 \%$ & Blue $10 \%$ \\
\hline & Brown $6 \%$ & Brown $10 \%$ \\
\hline & Red $8 \%$ & Red $4 \%$ \\
\hline & White $2 \%$ & White $2 \%$ \\
\hline & Yellow $2 \%$ & Purple $2 \%$ \\
\hline & Green $2 \%$ & Dark gray $2 \%$ \\
\hline & Orange $2 \%$ & $\mathrm{TSS}=8$ \\
\hline & $\mathrm{TSS}=9$ & $\mathrm{MSS}=5$ \\
\hline & $\mathrm{MSS}=5$ & \\
\hline \multirow[t]{10}{*}{ Tragedy } & Red $38 \%$ & Red $35 \%$ \\
\hline & Black $32 \%$ & Black $41 \%$ \\
\hline & Blue $10 \%$ & Blue $14 \%$ \\
\hline & Gray $10 \%$ & Gray $4 \%$ \\
\hline & Yellow $2 \%$ & Yellow $2 \%$ \\
\hline & White $4 \%$ & Purple $2 \%$ \\
\hline & Brown $2 \%$ & Orange $2 \%$ \\
\hline & Burgundy $2 \%$ & $\mathrm{TSS}=7$ \\
\hline & $\mathrm{TSS}=8$ & $\mathrm{MSS}=4$ \\
\hline & $\mathrm{MSS}=5$ & \\
\hline \multirow[t]{8}{*}{ Trash } & Black $46 \%$ & Black $53 \%$ \\
\hline & Gray $18 \%$ & Gray $15 \%$ \\
\hline & Brown $14 \%$ & Brown $12 \%$ \\
\hline & White $12 \%$ & White $8 \%$ \\
\hline & Green $8 \%$ & Green $10 \%$ \\
\hline & Dark green $2 \%$ & Maroon $2 \%$ \\
\hline & $\mathrm{TSS}=6$ & $\mathrm{TSS}=6$ \\
\hline & $\mathrm{MSS}=5$ & $\mathrm{MSS}=5$ \\
\hline Trauma & Red $53 \%$ & Red $49 \%$ \\
\hline
\end{tabular}

Table 7 (continued)

\begin{tabular}{|c|c|c|}
\hline Word & Sample A & Sample B \\
\hline \multirow{22}{*}{ Ugly } & Black $13 \%$ & Black $21 \%$ \\
\hline & White $6 \%$ & White $10 \%$ \\
\hline & Blue $10 \%$ & Blue $6 \%$ \\
\hline & Gray $10 \%$ & Gray $6 \%$ \\
\hline & Green $4 \%$ & Green $4 \%$ \\
\hline & Orange $2 \%$ & Purple $2 \%$ \\
\hline & Navy $2 \%$ & Dark blue $2 \%$ \\
\hline & $\mathrm{TSS}=8$ & $\mathrm{TSS}=8$ \\
\hline & $\mathrm{MSS}=6$ & $\mathrm{MSS}=6$ \\
\hline & Brown $38 \%$ & Brown $33 \%$ \\
\hline & Black $30 \%$ & Black $23 \%$ \\
\hline & Gray $6 \%$ & Gray $10 \%$ \\
\hline & Green $6 \%$ & Green $10 \%$ \\
\hline & White $6 \%$ & White $2 \%$ \\
\hline & Blue $4 \%$ & Blue $4 \%$ \\
\hline & Yellow $2 \%$ & Yellow $6 \%$ \\
\hline & Purple $2 \%$ & Purple $4 \%$ \\
\hline & Orange $2 \%$ & Orange $4 \%$ \\
\hline & Red $4 \%$ & Silver $2 \%$ \\
\hline & Chartreuse $2 \%$ & Fuchsia $2 \%$ \\
\hline & $\mathrm{TSS}=11$ & $\mathrm{TSS}=11$ \\
\hline & $\mathrm{MSS}=7$ & $\mathrm{MSS}=8$ \\
\hline \multirow[t]{13}{*}{ Ulcer } & Red $32 \%$ & Red $33 \%$ \\
\hline & Black $22 \%$ & Black $23 \%$ \\
\hline & Brown $10 \%$ & Brown $8 \%$ \\
\hline & White $8 \%$ & White $10 \%$ \\
\hline & Yellow $6 \%$ & Yellow $6 \%$ \\
\hline & Pink $4 \%$ & Pink $8 \%$ \\
\hline & Blue $6 \%$ & Blue $2 \%$ \\
\hline & Gray $4 \%$ & Gray $4 \%$ \\
\hline & Peach $2 \%$ & Peach $2 \%$ \\
\hline & Orange $4 \%$ & Clear $2 \%$ \\
\hline & Crimson $2 \%$ & Green $2 \%$ \\
\hline & $\mathrm{TSS}=11$ & $\mathrm{TSS}=11$ \\
\hline & $\mathrm{MSS}=9$ & $\mathrm{MSS}=7$ \\
\hline \multirow[t]{13}{*}{ Unfaithful } & Black $31 \%$ & Black $39 \%$ \\
\hline & Red $29 \%$ & Red $23 \%$ \\
\hline & Blue $10 \%$ & Blue $6 \%$ \\
\hline & Green $4 \%$ & Green $8 \%$ \\
\hline & Gray $8 \%$ & Gray $2 \%$ \\
\hline & Orange $4 \%$ & Orange $6 \%$ \\
\hline & Yellow $2 \%$ & Yellow $6 \%$ \\
\hline & White $4 \%$ & White $2 \%$ \\
\hline & Purple $2 \%$ & Purple $2 \%$ \\
\hline & Pink $4 \%$ & Brown $6 \%$ \\
\hline & Fire brick $2 \%$ & $\mathrm{TSS}=10$ \\
\hline & $\mathrm{TSS}=11$ & $\mathrm{MSS}=7$ \\
\hline & $\mathrm{MSS}=8$ & \\
\hline Victim & $\operatorname{Red} 46 \%$ & Red $40 \%$ \\
\hline
\end{tabular}


Table 7 (continued)

\begin{tabular}{|c|c|c|c|c|c|}
\hline Word & Sample A & Sample B & Word & Sample A & Sample B \\
\hline & Black $14 \%$ & Black $16 \%$ & & $\mathrm{MSS}=4$ & $\mathrm{MSS}=6$ \\
\hline & White $14 \%$ & White $10 \%$ & Aroused & Red $63 \%$ & Red $69 \%$ \\
\hline & Gray $12 \%$ & Gray $12 \%$ & & Pink $14 \%$ & Pink $9 \%$ \\
\hline & Blue $6 \%$ & Blue $10 \%$ & & Yellow $6 \%$ & Yellow $9 \%$ \\
\hline & Brown $2 \%$ & Purple $4 \%$ & & White $6 \%$ & White $4 \%$ \\
\hline & Pink $2 \%$ & Brown $2 \%$ & & Green $2 \%$ & Green $4 \%$ \\
\hline & Violet $2 \%$ & Yellow $2 \%$ & & Brown $2 \%$ & Brown $2 \%$ \\
\hline & Midnight blue $2 \%$ & Magenta $2 \%$ & & Black $4 \%$ & Blue-green $2 \%$ \\
\hline & $\mathrm{TSS}=9$ & Indigo $2 \%$ & & Orange $2 \%$ & $\mathrm{TSS}=7$ \\
\hline & $\mathrm{MSS}=5$ & $\mathrm{TSS}=10$ & & Hot pink $2 \%$ & $\mathrm{MSS}=5$ \\
\hline & & $\mathrm{MS}=6$ & & $\mathrm{TSS}=9$ & \\
\hline \multirow[t]{7}{*}{ Violent } & Red $58 \%$ & Red $63 \%$ & & $\mathrm{MSS}=5$ & \\
\hline & Black $22 \%$ & Black $23 \%$ & Astonished & Yellow $22 \%$ & Yellow $25 \%$ \\
\hline & Purple $10 \%$ & Purple $6 \%$ & & White $20 \%$ & White $22 \%$ \\
\hline & Violet $10 \%$ & Violet $4 \%$ & & Red $20 \%$ & Red $16 \%$ \\
\hline & $\mathrm{TSS}=4$ & Blue $4 \%$ & & Blue $10 \%$ & Blue $12 \%$ \\
\hline & & $\mathrm{MSS}=5$ & & Orange $12 \%$ & Orange $6 \%$ \\
\hline & $\mathrm{MSS}=4$ & $\mathrm{TSS}=5$ & & Green $4 \%$ & Green $8 \%$ \\
\hline \multirow[t]{9}{*}{ War } & $\operatorname{Red} 56 \%$ & Red $61 \%$ & & Black $4 \%$ & Black $2 \%$ \\
\hline & Black $10 \%$ & Black $12 \%$ & & Baby Blue $2 \%$ & Gray $4 \%$ \\
\hline & Green $14 \%$ & Green $6 \%$ & & Purple $2 \%$ & Brown $2 \%$ \\
\hline & Brown $10 \%$ & Brown $8 \%$ & & Cream/ivory $2 \%$ & Light blue $2 \%$ \\
\hline & Gray $8 \%$ & Gray $4 \%$ & & Chartreuse $2 \%$ & Milk white $2 \%$ \\
\hline & Blue $2 \%$ & Blue $6 \%$ & & $\mathrm{TSS}=11$ & $\mathrm{TSS}=11$ \\
\hline & $\mathrm{TSS}=6$ & Dark red $2 \%$ & & $\mathrm{MSS}=7$ & $\mathrm{MSS}=7$ \\
\hline & $\mathrm{MSS}=5$ & $\mathrm{TSS}=7$ & Beautiful & Pink $33 \%$ & Pink $37 \%$ \\
\hline & & $\mathrm{MSS}=6$ & & Blue $18 \%$ & Blue $22 \%$ \\
\hline Positive Emotion & & & & Yellow $12 \%$ & Yellow $6 \%$ \\
\hline \multirow[t]{13}{*}{ Admired } & Red $39 \%$ & Red $46 \%$ & & Purple $10 \%$ & Purple $6 \%$ \\
\hline & Blue $12 \%$ & Blue $16 \%$ & & White $4 \%$ & White $8 \%$ \\
\hline & Pink $15 \%$ & Pink $12 \%$ & & $\operatorname{Red} 4 \%$ & Red $8 \%$ \\
\hline & Yellow $14 \%$ & Yellow $10 \%$ & & Black $4 \%$ & Black $6 \%$ \\
\hline & Orange $4 \%$ & Orange $4 \%$ & & Green $6 \%$ & Green $2 \%$ \\
\hline & White $6 \%$ & White $2 \%$ & & Brown $4 \%$ & Gold $4 \%$ \\
\hline & Black $2 \%$ & Black $2 \%$ & & Sky blue $2 \%$ & Sky blue $2 \%$ \\
\hline & Green $2 \%$ & Green $2 \%$ & & Orange $2 \%$ & $\mathrm{TSS}=10$ \\
\hline & Light cyan $2 \%$ & Gold $4 \%$ & & Silver $2 \%$ & $\mathrm{MSS}=8$ \\
\hline & Silver $2 \%$ & Violet $2 \%$ & & $\mathrm{TSS}=12$ & \\
\hline & Sky blue $2 \%$ & $\mathrm{TSS}=10$ & & $\mathrm{MSS}=9$ & \\
\hline & $\mathrm{TSS}=11$ & $\mathrm{MSS}=6$ & Brave & Red $34 \%$ & Red $38 \%$ \\
\hline & $\mathrm{MSS}=6$ & & & Blue $30 \%$ & Blue $26 \%$ \\
\hline \multirow[t]{8}{*}{ Affection } & $\operatorname{Red} 51 \%$ & $\operatorname{Red} 45 \%$ & & Gold $6 \%$ & Gold $10 \%$ \\
\hline & Pink $29 \%$ & Pink $27 \%$ & & Yellow $6 \%$ & Yellow $4 \%$ \\
\hline & Yellow $6 \%$ & Yellow $10 \%$ & & Brown $8 \%$ & Brown $2 \%$ \\
\hline & Blue $8 \%$ & Blue $8 \%$ & & Black $4 \%$ & Black $4 \%$ \\
\hline & Purple $2 \%$ & Purple $4 \%$ & & White $2 \%$ & White $4 \%$ \\
\hline & Light blue $2 \%$ & White $4 \%$ & & Orange $2 \%$ & Orange $2 \%$ \\
\hline & Gold $2 \%$ & Green $2 \%$ & & Purple $4 \%$ & Purple $2 \%$ \\
\hline & $\mathrm{TSS}=7$ & $\mathrm{TSS}=7$ & & Silver $2 \%$ & Green $6 \%$ \\
\hline
\end{tabular}

Table 7 (continued) 
Table 7 (continued)

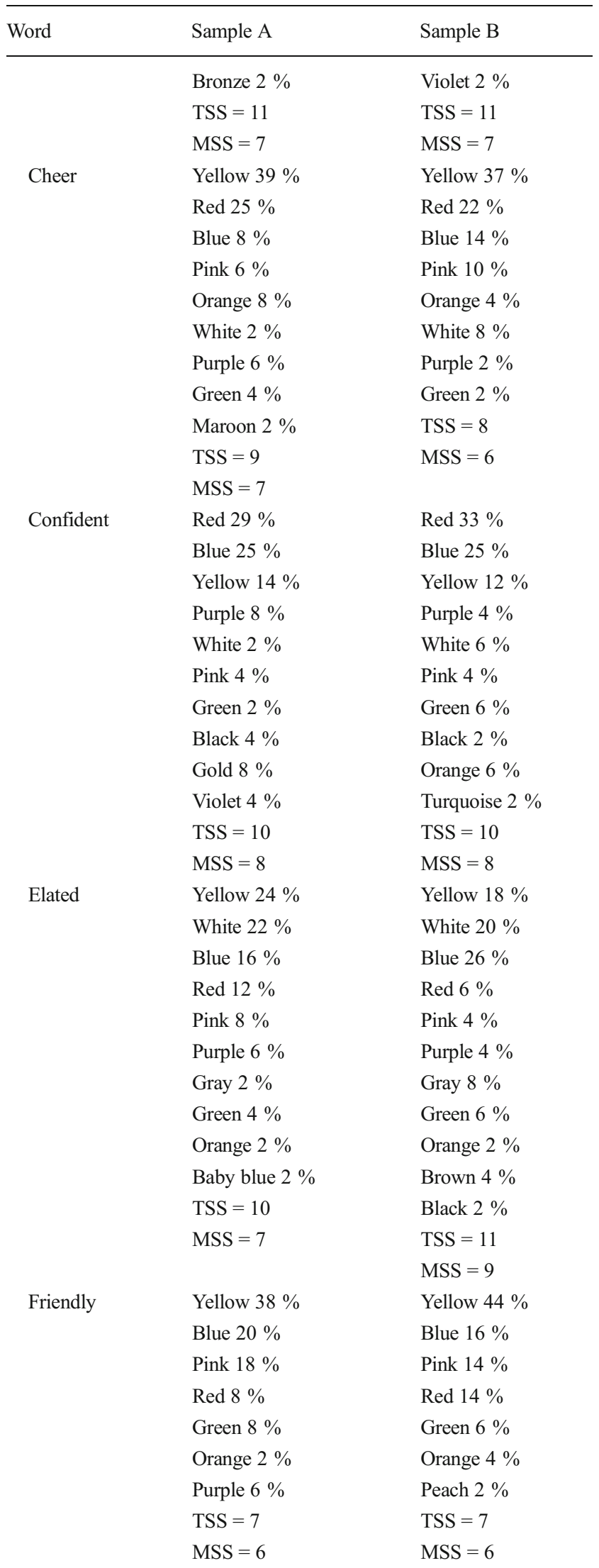

Table 7 (continued)

\begin{tabular}{|c|c|c|}
\hline Word & Sample A & Sample B \\
\hline \multirow[t]{15}{*}{ Grateful } & Blue $20 \%$ & Blue $23 \%$ \\
\hline & Yellow $16 \%$ & Yellow $18 \%$ \\
\hline & Green $16 \%$ & Green $14 \%$ \\
\hline & White $10 \%$ & White $6 \%$ \\
\hline & Purple $4 \%$ & Purple $12 \%$ \\
\hline & Red $8 \%$ & Red $6 \%$ \\
\hline & Pink $10 \%$ & Pink $4 \%$ \\
\hline & Orange $4 \%$ & Orange $8 \%$ \\
\hline & Gray $4 \%$ & Gray $6 \%$ \\
\hline & Lilac $2 \%$ & Beige $2 \%$ \\
\hline & Cream $2 \%$ & Lime green $2 \%$ \\
\hline & Coral $2 \%$ & $\mathrm{TSS}=11$ \\
\hline & Black $2 \%$ & $\mathrm{MSS}=9$ \\
\hline & $\mathrm{TSS}=13$ & \\
\hline & $\mathrm{MSS}=9$ & \\
\hline \multirow[t]{9}{*}{ Happy } & Yellow $54 \%$ & Yellow $52 \%$ \\
\hline & Red $14 \%$ & Red $20 \%$ \\
\hline & Pink $12 \%$ & Pink $10 \%$ \\
\hline & Blue $10 \%$ & Blue $6 \%$ \\
\hline & Green $4 \%$ & Green $8 \%$ \\
\hline & White $2 \%$ & White $2 \%$ \\
\hline & Purple $4 \%$ & Bright red $2 \%$ \\
\hline & $\mathrm{TSS}=7$ & $\mathrm{TSS}=7$ \\
\hline & $\mathrm{MSS}=6$ & $\mathrm{MSS}=5$ \\
\hline \multirow[t]{11}{*}{ Hopeful } & Blue $26 \%$ & Blue $33 \%$ \\
\hline & White $26 \%$ & White $31 \%$ \\
\hline & Yellow $22 \%$ & Yellow $15 \%$ \\
\hline & Pink $10 \%$ & Pink $8 \%$ \\
\hline & Red $8 \%$ & Red $2 \%$ \\
\hline & Orange $2 \%$ & Orange $4 \%$ \\
\hline & Periwinkle $2 \%$ & Green $2 \%$ \\
\hline & Light blue $2 \%$ & Magenta $2 \%$ \\
\hline & Silver $2 \%$ & Antique white $2 \%$ \\
\hline & $\mathrm{TSS}=9$ & $\mathrm{TSS}=9$ \\
\hline & $\mathrm{MSS}=5$ & $\mathrm{MSS}=5$ \\
\hline \multirow[t]{13}{*}{ Inspired } & Yellow $23 \%$ & Yellow $22 \%$ \\
\hline & White $17 \%$ & White $20 \%$ \\
\hline & Blue $21 \%$ & Blue $16 \%$ \\
\hline & Red $17 \%$ & Red $12 \%$ \\
\hline & Pink $4 \%$ & Pink $6 \%$ \\
\hline & Purple $2 \%$ & Purple $8 \%$ \\
\hline & Green $6 \%$ & Green $2 \%$ \\
\hline & Lavender $2 \%$ & Lavender $2 \%$ \\
\hline & Black $2 \%$ & Orange $4 \%$ \\
\hline & Violet $2 \%$ & Gold $2 \%$ \\
\hline & Peach $2 \%$ & Light blue $2 \%$ \\
\hline & Teal $2 \%$ & Blue-green $2 \%$ \\
\hline & $\mathrm{TSS}=12$ & Baby blue $2 \%$ \\
\hline
\end{tabular}


Table 7 (continued)

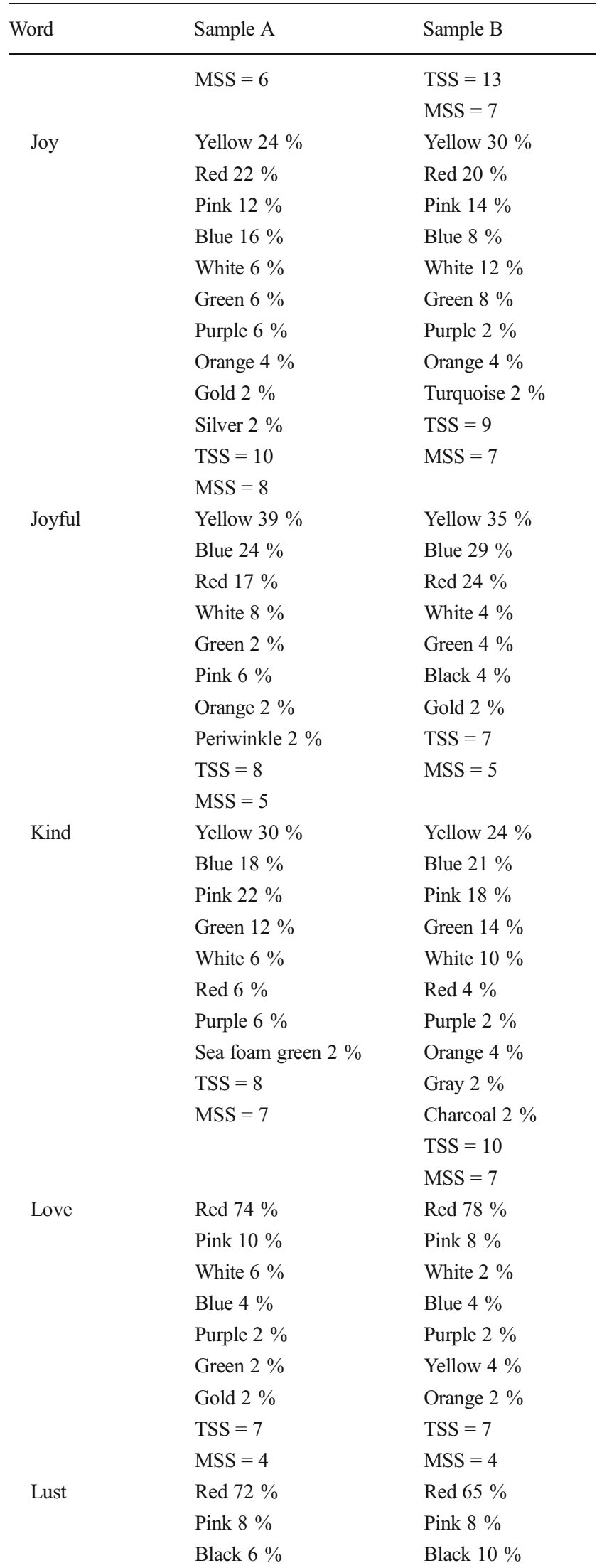

Table 7 (continued)

\begin{tabular}{|c|c|c|}
\hline Word & Sample A & Sample B \\
\hline \multirow{17}{*}{ Merry } & Purple $2 \%$ & Purple $6 \%$ \\
\hline & Yellow $2 \%$ & Yellow $4 \%$ \\
\hline & Orange $2 \%$ & Orange $2 \%$ \\
\hline & Violet $4 \%$ & Dark red $2 \%$ \\
\hline & Blue $2 \%$ & White $2 \%$ \\
\hline & Brown $2 \%$ & Green $2 \%$ \\
\hline & $\mathrm{TSS}=9$ & $\mathrm{TSS}=9$ \\
\hline & $\mathrm{MSS}=4$ & $\mathrm{MSS}=5$ \\
\hline & Red $66 \%$ & Red $63 \%$ \\
\hline & Green $14 \%$ & Green $12 \%$ \\
\hline & Yellow $8 \%$ & Yellow $12 \%$ \\
\hline & Blue $4 \%$ & Blue $8 \%$ \\
\hline & Pink 4 \% & Salmon $2 \%$ \\
\hline & Purple $2 \%$ & Gray $2 \%$ \\
\hline & Orange $2 \%$ & $\mathrm{TSS}=6$ \\
\hline & $\mathrm{TSS}=7$ & $\mathrm{MSS}=4$ \\
\hline & $\mathrm{MSS}=5$ & \\
\hline \multirow[t]{13}{*}{ Nice } & Yellow $26 \%$ & Yellow $33 \%$ \\
\hline & Blue $26 \%$ & Blue $22 \%$ \\
\hline & Pink $16 \%$ & Pink $17 \%$ \\
\hline & White $10 \%$ & White $16 \%$ \\
\hline & Red $8 \%$ & Red $2 \%$ \\
\hline & Purple $2 \%$ & Purple $2 \%$ \\
\hline & Black $2 \%$ & Green $4 \%$ \\
\hline & Gold $4 \%$ & Black $2 \%$ \\
\hline & Silver $2 \%$ & Light blue $2 \%$ \\
\hline & Lilac $2 \%$ & $\mathrm{TSS}=9$ \\
\hline & Indigo $2 \%$ & $\mathrm{MSS}=5$ \\
\hline & $\mathrm{TSS}=11$ & \\
\hline & $\mathrm{MSS}=6$ & \\
\hline \multirow[t]{10}{*}{ Passion } & Red $79 \%$ & Red $76 \%$ \\
\hline & Pink $4 \%$ & Pink $8 \%$ \\
\hline & White $6 \%$ & White $2 \%$ \\
\hline & Blue $4 \%$ & Blue $4 \%$ \\
\hline & Yellow 4 \% & Yellow $2 \%$ \\
\hline & Dark red $2 \%$ & Purple $4 \%$ \\
\hline & Scarlett $2 \%$ & Green $2 \%$ \\
\hline & $\mathrm{TSS}=7$ & Black $2 \%$ \\
\hline & $\mathrm{MSS}=5$ & $\mathrm{TSS}=8$ \\
\hline & & $\mathrm{MSS}=4$ \\
\hline \multirow[t]{8}{*}{ Proud } & Blue $24 \%$ & Blue $31 \%$ \\
\hline & Red $22 \%$ & Red $20 \%$ \\
\hline & Yellow $18 \%$ & Yellow $14 \%$ \\
\hline & White $8 \%$ & White $12 \%$ \\
\hline & Purple $10 \%$ & Purple $6 \%$ \\
\hline & Orange $4 \%$ & Orange $6 \%$ \\
\hline & Green $2 \%$ & Green $6 \%$ \\
\hline & Black $4 \%$ & Gold $4 \%$ \\
\hline
\end{tabular}


Table 7 (continued)

\begin{tabular}{|c|c|c|}
\hline Word & Sample A & Sample B \\
\hline \multirow{20}{*}{ Relaxed } & Pink $4 \%$ & Beige $2 \%$ \\
\hline & Peach $2 \%$ & $\mathrm{TSS}=10$ \\
\hline & Misty rose $2 \%$ & $\mathrm{MSS}=8$ \\
\hline & $\mathrm{TSS}=11$ & \\
\hline & $\mathrm{MSS}=8$ & \\
\hline & Blue $42 \%$ & Blue $42 \%$ \\
\hline & White $15 \%$ & White $12 \%$ \\
\hline & Yellow $8 \%$ & Yellow $16 \%$ \\
\hline & Pink $8 \%$ & Pink $4 \%$ \\
\hline & Green $9 \%$ & Green $2 \%$ \\
\hline & Red $4 \%$ & Red $4 \%$ \\
\hline & Light blue $2 \%$ & Light blue $4 \%$ \\
\hline & Sky blue $4 \%$ & Purple $6 \%$ \\
\hline & $\operatorname{Tan}(1) 2 \%$ & Tan $2 \%$ \\
\hline & Lavender $2 \%$ & Baby blue $2 \%$ \\
\hline & Gray $2 \%$ & Peach $2 \%$ \\
\hline & Brown $2 \%$ & Off white $2 \%$ \\
\hline & $\mathrm{TSS}=12$ & Forest green $2 \%$ \\
\hline & $\mathrm{MSS}=7$ & $\mathrm{TSS}=13$ \\
\hline & & $\mathrm{MSS}=7$ \\
\hline \multirow[t]{13}{*}{ Safe } & White $29 \%$ & White $32 \%$ \\
\hline & Blue $33 \%$ & Blue $26 \%$ \\
\hline & Yellow $6 \%$ & Yellow $13 \%$ \\
\hline & Green $10 \%$ & Green $6 \%$ \\
\hline & Gray $4 \%$ & Gray $7 \%$ \\
\hline & Red $6 \%$ & Red $4 \%$ \\
\hline & Black $2 \%$ & Black $6 \%$ \\
\hline & Pink $4 \%$ & Purple $2 \%$ \\
\hline & Orange $2 \%$ & Brown $2 \%$ \\
\hline & Gold $2 \%$ & Pale blue $2 \%$ \\
\hline & Beige $2 \%$ & $\mathrm{TSS}=10$ \\
\hline & $\mathrm{TSS}=11$ & \\
\hline & $\mathrm{MSS}=7$ & $\mathrm{MSS}=7$ \\
\hline \multirow[t]{12}{*}{ Satisfied } & Blue $36 \%$ & Blue $30 \%$ \\
\hline & Red $20 \%$ & Red $16 \%$ \\
\hline & Yellow $14 \%$ & Yellow $14 \%$ \\
\hline & White $8 \%$ & White $14 \%$ \\
\hline & Green $6 \%$ & Green $12 \%$ \\
\hline & Pink $4 \%$ & Pink $10 \%$ \\
\hline & Orange $6 \%$ & Orange $2 \%$ \\
\hline & Lime green $2 \%$ & Black $2 \%$ \\
\hline & Gold $2 \%$ & $\mathrm{TSS}=8$ \\
\hline & Purple $2 \%$ & $\mathrm{MSS}=6$ \\
\hline & $\mathrm{TSS}=10$ & \\
\hline & $\mathrm{MSS}=7$ & \\
\hline \multirow[t]{4}{*}{ Secure } & Blue $24 \%$ & Blue $30 \%$ \\
\hline & White $25 \%$ & White $20 \%$ \\
\hline & Red $16 \%$ & Red $12 \%$ \\
\hline & Yellow $12 \%$ & Yellow $6 \%$ \\
\hline
\end{tabular}

Table 7 (continued)

\begin{tabular}{|c|c|c|}
\hline Word & Sample A & Sample B \\
\hline & Green $6 \%$ & Green $10 \%$ \\
\hline & Gray $4 \%$ & Gray $6 \%$ \\
\hline & Black $2 \%$ & Black $4 \%$ \\
\hline & Silver $4 \%$ & Gold $6 \%$ \\
\hline & Sapphire $2 \%$ & Pink $2 \%$ \\
\hline & Light blue $2 \%$ & Salmon $2 \%$ \\
\hline & Purple $2 \%$ & Orange $2 \%$ \\
\hline & Brown $2 \%$ & $\mathrm{TSS}=11$ \\
\hline & $\mathrm{TSS}=12$ & $\mathrm{MSS}=8$ \\
\hline & $\mathrm{MSS}=7$ & \\
\hline \multirow[t]{12}{*}{ Surprised } & Red $31 \%$ & Red $33 \%$ \\
\hline & Yellow $24 \%$ & Yellow $29 \%$ \\
\hline & White $10 \%$ & White $8 \%$ \\
\hline & Blue $10 \%$ & Blue $6 \%$ \\
\hline & Orange $8 \%$ & Orange $8 \%$ \\
\hline & Pink 4 \% & Pink $10 \%$ \\
\hline & Green $4 \%$ & Green $2 \%$ \\
\hline & Purple $4 \%$ & Violet $2 \%$ \\
\hline & Black $2 \%$ & Silver $2 \%$ \\
\hline & Gold $2 \%$ & $\mathrm{TSS}=9$ \\
\hline & $\mathrm{TSS}=10$ & $\mathrm{MSS}=6$ \\
\hline & $\mathrm{MSS}=8$ & \\
\hline \multirow[t]{14}{*}{ Triumphant } & Yellow $27 \%$ & Yellow $24 \%$ \\
\hline & Red $25 \%$ & Red $18 \%$ \\
\hline & Blue $8 \%$ & Blue $14 \%$ \\
\hline & Gold $6 \%$ & Gold $14 \%$ \\
\hline & White $8 \%$ & White $4 \%$ \\
\hline & Purple $2 \%$ & Purple $8 \%$ \\
\hline & Green $6 \%$ & Green $2 \%$ \\
\hline & Black $4 \%$ & Black $4 \%$ \\
\hline & Brown $2 \%$ & Brown $4 \%$ \\
\hline & Pink $2 \%$ & Pink $2 \%$ \\
\hline & Gray $6 \%$ & Orange $4 \%$ \\
\hline & Silver $4 \%$ & Bronze $2 \%$ \\
\hline & $\mathrm{TSS}=11$ & $\mathrm{TSS}=12$ \\
\hline & $\mathrm{MSS}=8$ & $\mathrm{MSS}=9$ \\
\hline \multicolumn{3}{|c|}{ Positive Emotion-Laden } \\
\hline \multirow[t]{9}{*}{ Achievement } & Blue $30 \%$ & Blue $24 \%$ \\
\hline & Gold $32 \%$ & Gold $22 \%$ \\
\hline & Red $14 \%$ & Red $18 \%$ \\
\hline & Yellow $12 \%$ & Yellow $20 \%$ \\
\hline & Green $8 \%$ & Green $4 \%$ \\
\hline & White $2 \%$ & White $8 \%$ \\
\hline & Purple $2 \%$ & Orange $4 \%$ \\
\hline & $\mathrm{TSS}=7$ & $\mathrm{TSS}=7$ \\
\hline & $\mathrm{MSS}=5$ & $\mathrm{MSS}=7$ \\
\hline \multirow[t]{3}{*}{ Adorable } & Pink 44 \% & Pink 54 \% \\
\hline & $\operatorname{Red} 23 \%$ & Red $20 \%$ \\
\hline & Blue $15 \%$ & Blue $8 \%$ \\
\hline
\end{tabular}


Table 7 (continued)

\begin{tabular}{|c|c|c|}
\hline Word & Sample A & Sample B \\
\hline & Yellow $4 \%$ & Yellow $8 \%$ \\
\hline & Green $6 \%$ & Green $2 \%$ \\
\hline & Brown $2 \%$ & Brown $2 \%$ \\
\hline & Purple $2 \%$ & White $4 \%$ \\
\hline & Tan $2 \%$ & Light blue $2 \%$ \\
\hline & Lavender $2 \%$ & $\mathrm{TSS}=8$ \\
\hline & $\mathrm{TSS}=9$ & $\mathrm{MSS}=5$ \\
\hline & $\mathrm{MSS}=5$ & \\
\hline \multirow[t]{11}{*}{ Baby } & Pink $42 \%$ & Pink $34 \%$ \\
\hline & White $18 \%$ & White $23 \%$ \\
\hline & Blue $18 \%$ & Blue $21 \%$ \\
\hline & Yellow $6 \%$ & Yellow 7 \% \\
\hline & Peach $8 \%$ & Peach $4 \%$ \\
\hline & Black $2 \%$ & Black $6 \%$ \\
\hline & Brown $2 \%$ & Brown $2 \%$ \\
\hline & Green $2 \%$ & Red $4 \%$ \\
\hline & Dodger blue $2 \%$ & $\mathrm{TSS}=8$ \\
\hline & $\mathrm{TSS}=9$ & $\mathrm{MSS}=7$ \\
\hline & $\mathrm{MSS}=5$ & \\
\hline \multirow[t]{11}{*}{ Birthday } & Blue $19 \%$ & Blue $28 \%$ \\
\hline & Pink $19 \%$ & Pink $22 \%$ \\
\hline & Yellow $23 \%$ & Yellow $16 \%$ \\
\hline & Red $13 \%$ & Red $18 \%$ \\
\hline & White $10 \%$ & White $4 \%$ \\
\hline & Green $8 \%$ & Green $2 \%$ \\
\hline & Purple $2 \%$ & Purple $6 \%$ \\
\hline & Black $4 \%$ & Black $2 \%$ \\
\hline & Orange $2 \%$ & Magenta $2 \%$ \\
\hline & $\mathrm{TSS}=9$ & $\mathrm{TSS}=9$ \\
\hline & $\mathrm{MSS}=7$ & $\mathrm{MSS}=6$ \\
\hline \multirow[t]{11}{*}{ Bouquet } & Red $50 \%$ & Red $43 \%$ \\
\hline & Pink $18 \%$ & Pink $25 \%$ \\
\hline & White $10 \%$ & White $6 \%$ \\
\hline & Green $12 \%$ & Green $4 \%$ \\
\hline & Yellow $6 \%$ & Yellow $6 \%$ \\
\hline & Blue $2 \%$ & Blue $8 \%$ \\
\hline & Purple $2 \%$ & Purple $4 \%$ \\
\hline & $\mathrm{TSS}=7$ & Brown $2 \%$ \\
\hline & $\mathrm{MSS}=5$ & Orange $2 \%$ \\
\hline & & $\mathrm{TSS}=9$ \\
\hline & & $\mathrm{MSS}=7$ \\
\hline \multirow[t]{7}{*}{ Cash } & Green $90 \%$ & Green $96 \%$ \\
\hline & Black $2 \%$ & Black $2 \%$ \\
\hline & Gray $4 \%$ & White $2 \%$ \\
\hline & Gold $2 \%$ & $\mathrm{TSS}=3$ \\
\hline & Brown $2 \%$ & $\mathrm{MSS}=1$ \\
\hline & $\mathrm{TSS}=5$ & \\
\hline & $\mathrm{MSS}=2$ & \\
\hline Champion & Gold $39 \%$ & Gold $34 \%$ \\
\hline
\end{tabular}

Table 7 (continued)

\begin{tabular}{|c|c|c|}
\hline Word & Sample A & Sample B \\
\hline & Red $23 \%$ & Red $20 \%$ \\
\hline & Yellow $19 \%$ & Yellow $24 \%$ \\
\hline & Blue $8 \%$ & Blue $16 \%$ \\
\hline & White $6 \%$ & White $2 \%$ \\
\hline & Purple $2 \%$ & Orange $2 \%$ \\
\hline & Black $2 \%$ & Silver $2 \%$ \\
\hline & $\mathrm{TSS}=7$ & $\mathrm{TSS}=7$ \\
\hline & $\mathrm{MSS}=5$ & $\mathrm{MSS}=4$ \\
\hline \multirow[t]{10}{*}{ Church } & White $58 \%$ & White $64 \%$ \\
\hline & Brown $14 \%$ & Brown $18 \%$ \\
\hline & Gray $8 \%$ & Gray $4 \%$ \\
\hline & Red $6 \%$ & Red $6 \%$ \\
\hline & Black $6 \%$ & Black $2 \%$ \\
\hline & Yellow 4 \% & Yellow $2 \%$ \\
\hline & Pink $2 \%$ & Purple $4 \%$ \\
\hline & Gold $2 \%$ & $\mathrm{TSS}=7$ \\
\hline & $\mathrm{TSS}=8$ & $\mathrm{MSS}=5$ \\
\hline & $\mathrm{MSS}=6$ & \\
\hline \multirow[t]{11}{*}{ Diamond } & White $76 \%$ & White $65 \%$ \\
\hline & Silver $6 \%$ & Silver $10 \%$ \\
\hline & Clear $4 \%$ & Clear $8 \%$ \\
\hline & Blue $4 \%$ & Blue $2 \%$ \\
\hline & Yellow $2 \%$ & Yellow 4 \% \\
\hline & Red $2 \%$ & Red $4 \%$ \\
\hline & Gold $6 \%$ & Black $4 \%$ \\
\hline & $\mathrm{TSS}=7$ & Pink $2 \%$ \\
\hline & $\mathrm{MSS}=5$ & Purple $2 \%$ \\
\hline & & $\mathrm{TSS}=9$ \\
\hline & & $\mathrm{MSS}=6$ \\
\hline \multirow[t]{12}{*}{ Diploma } & White $69 \%$ & White $67 \%$ \\
\hline & Blue $10 \%$ & Blue $8 \%$ \\
\hline & Black $4 \%$ & Black $10 \%$ \\
\hline & Gold $4 \%$ & Gold $2 \%$ \\
\hline & Cream 4 \% & Red $6 \%$ \\
\hline & Gray $2 \%$ & Yellow $2 \%$ \\
\hline & Purple $2 \%$ & Green $2 \%$ \\
\hline & Tan $2 \%$ & Silver $2 \%$ \\
\hline & Copper $2 \%$ & $\mathrm{TSS}=9$ \\
\hline & Lime green $2 \%$ & $\mathrm{MSS}=4$ \\
\hline & $\mathrm{TSS}=10$ & \\
\hline & $\mathrm{MSS}=5$ & \\
\hline \multirow[t]{8}{*}{ Engaged } & White $29 \%$ & White $38 \%$ \\
\hline & Blue $13 \%$ & Blue $17 \%$ \\
\hline & Pink $15 \%$ & Pink $8 \%$ \\
\hline & Gold $10 \%$ & Gold $15 \%$ \\
\hline & Red $11 \%$ & Red $6 \%$ \\
\hline & Silver $10 \%$ & Silver $8 \%$ \\
\hline & Yellow $10 \%$ & Yellow 4 \% \\
\hline & Light blue $2 \%$ & Purple $2 \%$ \\
\hline
\end{tabular}


Table 7 (continued)

\begin{tabular}{|c|c|c|}
\hline Word & Sample A & Sample B \\
\hline & $\mathrm{TSS}=8$ & Orange $2 \%$ \\
\hline & $\mathrm{MSS}=7$ & $\mathrm{TSS}=9$ \\
\hline & & $\mathrm{MSS}=7$ \\
\hline \multirow[t]{11}{*}{ Freedom } & White $33 \%$ & White $26 \%$ \\
\hline & Blue $25 \%$ & Blue $30 \%$ \\
\hline & Red $20 \%$ & Red $14 \%$ \\
\hline & Yellow 8 \% & Yellow $8 \%$ \\
\hline & Green $4 \%$ & Green $10 \%$ \\
\hline & Black $2 \%$ & Black $6 \%$ \\
\hline & Brown $4 \%$ & Orange $2 \%$ \\
\hline & Forrest green $2 \%$ & Gold $2 \%$ \\
\hline & Royal blue $2 \%$ & Silver $2 \%$ \\
\hline & $\mathrm{TSS}=9$ & $\mathrm{TSS}=9$ \\
\hline & $\mathrm{MSS}=6$ & $\mathrm{MSS}=6$ \\
\hline \multirow[t]{13}{*}{ Friend } & Yellow $24 \%$ & Yellow $31 \%$ \\
\hline & Blue $26 \%$ & Blue $22 \%$ \\
\hline & Pink $16 \%$ & Pink $12 \%$ \\
\hline & Green $8 \%$ & Green $10 \%$ \\
\hline & Red $8 \%$ & Red $4 \%$ \\
\hline & Purple $4 \%$ & Purple $6 \%$ \\
\hline & White $2 \%$ & White $6 \%$ \\
\hline & Brown $4 \%$ & Brown $2 \%$ \\
\hline & Gray $2 \%$ & Gray $2 \%$ \\
\hline & Black $4 \%$ & Orange $2 \%$ \\
\hline & Dark green $2 \%$ & Gold $2 \%$ \\
\hline & $\mathrm{TSS}=11$ & $\mathrm{TSS}=11$ \\
\hline & $\mathrm{MSS}=8$ & $\mathrm{MSS}=7$ \\
\hline \multirow[t]{13}{*}{ Gift } & Blue $27 \%$ & Blue $33 \%$ \\
\hline & $\operatorname{Red} 25 \%$ & Red $19 \%$ \\
\hline & Green $16 \%$ & Green $9 \%$ \\
\hline & Yellow $6 \%$ & Yellow $9 \%$ \\
\hline & Pink (5) $10 \%$ & Pink 4 \% \\
\hline & White (1)2\% & White $8 \%$ \\
\hline & Purple (2)4\% & Purple $6 \%$ \\
\hline & Gold (2)4 \% & Gold $4 \%$ \\
\hline & Orange (1) $2 \%$ & Orange $4 \%$ \\
\hline & Silver (2)4\% & Black $2 \%$ \\
\hline & $\mathrm{TSS}=10$ & Sapphire $2 \%$ \\
\hline & $\mathrm{MSS}=8$ & $\mathrm{TSS}=11$ \\
\hline & & $\mathrm{MSS}=9$ \\
\hline \multirow[t]{9}{*}{ God } & White $76 \%$ & White $77 \%$ \\
\hline & Black $4 \%$ & Black $4 \%$ \\
\hline & Gold $4 \%$ & Gold $9 \%$ \\
\hline & Red $6 \%$ & Green $4 \%$ \\
\hline & Blue $4 \%$ & Navy $2 \%$ \\
\hline & Yellow $2 \%$ & Gray $2 \%$ \\
\hline & Purple $2 \%$ & Brown $2 \%$ \\
\hline & Bronze $2 \%$ & $\mathrm{TSS}=7$ \\
\hline & $\mathrm{TSS}=8$ & $\mathrm{MSS}=4$ \\
\hline
\end{tabular}

Table 7 (continued)

\begin{tabular}{|c|c|c|}
\hline Word & Sample A & Sample B \\
\hline & $\mathrm{MSS}=5$ & \\
\hline \multirow[t]{13}{*}{ Grin } & White $32 \%$ & White $23 \%$ \\
\hline & Yellow $20 \%$ & Yellow $21 \%$ \\
\hline & Red $16 \%$ & Red $19 \%$ \\
\hline & Green $8 \%$ & Green $13 \%$ \\
\hline & Pink $10 \%$ & Pink $6 \%$ \\
\hline & Blue $2 \%$ & Blue $8 \%$ \\
\hline & Gray $4 \%$ & Gray $2 \%$ \\
\hline & Orange $2 \%$ & Orange $2 \%$ \\
\hline & Peach $2 \%$ & Black $4 \%$ \\
\hline & Purple $2 \%$ & $\operatorname{Tan} 2 \%$ \\
\hline & Brown $2 \%$ & $\mathrm{TSS}=10$ \\
\hline & $\mathrm{TSS}=11$ & $\mathrm{MSS}=7$ \\
\hline & $\mathrm{MSS}=6$ & \\
\hline \multirow[t]{9}{*}{ Heaven } & White $68 \%$ & White $76 \%$ \\
\hline & Blue $18 \%$ & Blue $14 \%$ \\
\hline & Yellow 6 \% & Yellow $2 \%$ \\
\hline & Gold $2 \%$ & Gold $4 \%$ \\
\hline & Green $2 \%$ & Black $2 \%$ \\
\hline & Silver $2 \%$ & Orange $2 \%$ \\
\hline & Black and white $2 \%$ & $\mathrm{TSS}=6$ \\
\hline & $\mathrm{TSS}=7$ & $\mathrm{MSS}=3$ \\
\hline & $\mathrm{MSS}=3$ & \\
\hline \multirow[t]{10}{*}{ Holiday } & Red $47 \%$ & $\operatorname{Red} 42 \%$ \\
\hline & Green $31 \%$ & Green $26 \%$ \\
\hline & Yellow $8 \%$ & Yellow $10 \%$ \\
\hline & White $4 \%$ & White $8 \%$ \\
\hline & Blue $6 \%$ & Blue $6 \%$ \\
\hline & Brown $2 \%$ & Brown $2 \%$ \\
\hline & Gold $2 \%$ & Pink $4 \%$ \\
\hline & $\mathrm{TSS}=7$ & Orange $2 \%$ \\
\hline & $\mathrm{MSS}=5$ & $\mathrm{TSS}=8$ \\
\hline & & $\mathrm{MSS}=6$ \\
\hline \multirow[t]{15}{*}{ Jewel } & Red $18 \%$ & Red $23 \%$ \\
\hline & White $16 \%$ & White $18 \%$ \\
\hline & Blue $20 \%$ & Blue $14 \%$ \\
\hline & Gold $8 \%$ & Gold $16 \%$ \\
\hline & Green $10 \%$ & Green $6 \%$ \\
\hline & Purple $12 \%$ & Purple $4 \%$ \\
\hline & Pink $4 \%$ & Pink $6 \%$ \\
\hline & Yellow $2 \%$ & Yellow $2 \%$ \\
\hline & Silver $2 \%$ & Silver $2 \%$ \\
\hline & Orange $4 \%$ & Clear $4 \%$ \\
\hline & Golden red $2 \%$ & Gray $2 \%$ \\
\hline & Ruby $2 \%$ & Lavender $2 \%$ \\
\hline & $\mathrm{TSS}=12$ & Sapphire $2 \%$ \\
\hline & $\mathrm{MSS}=8$ & $\mathrm{TSS}=13$ \\
\hline & & $\mathrm{MSS}=8$ \\
\hline Joke & Yellow 27 \% & Yellow $23 \%$ \\
\hline
\end{tabular}


Table 7 (continued)

\begin{tabular}{|c|c|c|}
\hline Word & Sample A & Sample B \\
\hline & Blue $22 \%$ & Blue $19 \%$ \\
\hline & Red $16 \%$ & $\operatorname{Red} 21 \%$ \\
\hline & Green $14 \%$ & Green $17 \%$ \\
\hline & Orange $8 \%$ & Orange $4 \%$ \\
\hline & Pink $6 \%$ & Pink $6 \%$ \\
\hline & White $4 \%$ & White $2 \%$ \\
\hline & Black $2 \%$ & Black $2 \%$ \\
\hline & Magenta $2 \%$ & Purple $4 \%$ \\
\hline & $\mathrm{TSS}=9$ & Azure $2 \%$ \\
\hline & $\mathrm{MSS}=7$ & $\mathrm{TSS}=10$ \\
\hline & & $\mathrm{MSS}=7$ \\
\hline \multirow[t]{8}{*}{ Kiss } & Red $59 \%$ & Red $67 \%$ \\
\hline & Pink $29 \%$ & Pink $22 \%$ \\
\hline & Blue $8 \%$ & Blue $2 \%$ \\
\hline & Yellow $2 \%$ & White $4 \%$ \\
\hline & Brown $2 \%$ & Black $2 \%$ \\
\hline & $\mathrm{TSS}=5$ & Light pink $2 \%$ \\
\hline & $\mathrm{MSS}=3$ & $\mathrm{TSS}=6$ \\
\hline & & $\mathrm{MSS}=3$ \\
\hline \multirow[t]{11}{*}{ Laughter } & Yellow $45 \%$ & Yellow $39 \%$ \\
\hline & Red $12 \%$ & Red $19 \%$ \\
\hline & Blue $12 \%$ & Blue $18 \%$ \\
\hline & Pink $12 \%$ & Pink $6 \%$ \\
\hline & White $6 \%$ & White $10 \%$ \\
\hline & Green $4 \%$ & Green $2 \%$ \\
\hline & Purple $4 \%$ & Purple $2 \%$ \\
\hline & Orange $2 \%$ & Gold $2 \%$ \\
\hline & Sky blue $2 \%$ & Peach orange $2 \%$ \\
\hline & $\mathrm{TSS}=9$ & $\mathrm{TSS}=9$ \\
\hline & $\mathrm{MSS}=7$ & $\mathrm{MSS}=5$ \\
\hline \multirow[t]{12}{*}{ Lucky } & Green $52 \%$ & Green $49 \%$ \\
\hline & Yellow $19 \%$ & Yellow $24 \%$ \\
\hline & Blue $10 \%$ & Blue $4 \%$ \\
\hline & Red $6 \%$ & Red $4 \%$ \\
\hline & White $2 \%$ & White $6 \%$ \\
\hline & Pink $2 \%$ & Pink $4 \%$ \\
\hline & Gold $4 \%$ & Gold $2 \%$ \\
\hline & Gray $2 \%$ & Gray $2 \%$ \\
\hline & Black $2 \%$ & Purple $2 \%$ \\
\hline & Orange $2 \%$ & Dark green $2 \%$ \\
\hline & $\mathrm{TSS}=10$ & $\mathrm{TSS}=10$ \\
\hline & $\mathrm{MSS}=5$ & $\mathrm{MSS}=6$ \\
\hline \multirow[t]{7}{*}{ Millionaire } & Green $82 \%$ & Green $86 \%$ \\
\hline & White $6 \%$ & White $2 \%$ \\
\hline & Blue $2 \%$ & Blue $6 \%$ \\
\hline & Gold $2 \%$ & Gold $4 \%$ \\
\hline & Red $4 \%$ & Silver $2 \%$ \\
\hline & Dark green $2 \%$ & $\mathrm{TSS}=5$ \\
\hline & Purple $2 \%$ & $\mathrm{MSS}=3$ \\
\hline
\end{tabular}

Table 7 (continued)

\begin{tabular}{|c|c|c|}
\hline Word & Sample A & Sample B \\
\hline \multirow{14}{*}{ Miracle } & $\mathrm{TSS}=7$ & \\
\hline & $\mathrm{MSS}=3$ & \\
\hline & White $46 \%$ & White $49 \%$ \\
\hline & Blue $16 \%$ & Blue $20 \%$ \\
\hline & Yellow $12 \%$ & Yellow $6 \%$ \\
\hline & Gold $8 \%$ & Gold $10 \%$ \\
\hline & Pink $6 \%$ & Pink $4 \%$ \\
\hline & Red $2 \%$ & Red $6 \%$ \\
\hline & Green $4 \%$ & Silver $2 \%$ \\
\hline & Off-White $2 \%$ & Orange $2 \%$ \\
\hline & Aqua $2 \%$ & Purple $2 \%$ \\
\hline & Gray $2 \%$ & $\mathrm{TSS}=9$ \\
\hline & $\mathrm{TSS}=10$ & $\mathrm{MSS}=6$ \\
\hline & $\mathrm{MSS}=6$ & \\
\hline \multirow[t]{5}{*}{ Money } & Green $100 \%$ & Green $96 \%$ \\
\hline & $\mathrm{TSS}=1$ & Brown $2 \%$ \\
\hline & $\mathrm{MSS}=1$ & Indigo $2 \%$ \\
\hline & & $\mathrm{TSS}=3$ \\
\hline & & $\mathrm{MSS}=1$ \\
\hline \multirow[t]{9}{*}{ Outdoors } & Green $65 \%$ & Green $62 \%$ \\
\hline & Blue $23 \%$ & Blue $16 \%$ \\
\hline & White $6 \%$ & White $4 \%$ \\
\hline & Yellow $2 \%$ & Yellow $8 \%$ \\
\hline & Gray $2 \%$ & Brown $6 \%$ \\
\hline & Forrest Green $2 \%$ & Sky Blue $2 \%$ \\
\hline & $\mathrm{TSS}=6$ & Red $2 \%$ \\
\hline & $\mathrm{MSS}=3$ & $\mathrm{TSS}=7$ \\
\hline & & $\mathrm{MSS}=5$ \\
\hline \multirow[t]{10}{*}{ Peace } & White $41 \%$ & White $47 \%$ \\
\hline & Blue $25 \%$ & Blue $31 \%$ \\
\hline & Yellow $10 \%$ & Yellow $6 \%$ \\
\hline & Green $10 \%$ & Green $6 \%$ \\
\hline & Pink $6 \%$ & Pink $2 \%$ \\
\hline & Orange $4 \%$ & Red $4 \%$ \\
\hline & Sky Blue $2 \%$ & Light Blue $2 \%$ \\
\hline & Purple $2 \%$ & Gold $2 \%$ \\
\hline & $\mathrm{TSS}=8$ & $\mathrm{TSS}=8$ \\
\hline & $\mathrm{MSS}=6$ & $\mathrm{MSS}=5$ \\
\hline \multirow[t]{10}{*}{ Pretty } & Pink $67 \%$ & Pink $59 \%$ \\
\hline & Red $6 \%$ & Red $12 \%$ \\
\hline & Blue $8 \%$ & Blue $4 \%$ \\
\hline & Black $4 \%$ & Black $8 \%$ \\
\hline & Yellow 8 \% & Yellow $2 \%$ \\
\hline & Purple 4 \% & Purple $6 \%$ \\
\hline & Green $2 \%$ & White $8 \%$ \\
\hline & Brown $2 \%$ & Light Pink $2 \%$ \\
\hline & $\mathrm{TSS}=8$ & $\mathrm{TSS}=8$ \\
\hline & $\mathrm{MSS}=6$ & $\mathrm{MSS}=6$ \\
\hline Rescue & Red $60 \%$ & Red $65 \%$ \\
\hline
\end{tabular}


Table 7 (continued)

\begin{tabular}{|c|c|c|c|c|c|}
\hline Word & Sample A & Sample B & Word & Sample A & Sample B \\
\hline & Blue $19 \%$ & Blue $12 \%$ & & White $8 \%$ & White $4 \%$ \\
\hline & Yellow $10 \%$ & Yellow $6 \%$ & & Green $2 \%$ & Green $2 \%$ \\
\hline & White $2 \%$ & White $8 \%$ & & Black $4 \%$ & Gray $2 \%$ \\
\hline & Brown $2 \%$ & Brown $2 \%$ & & Purple $2 \%$ & Brown $2 \%$ \\
\hline & Black $6 \%$ & Green $4 \%$ & & Bronze $2 \%$ & Silver $2 \%$ \\
\hline & $\mathrm{TSS}=6$ & Tan $2 \%$ & & $\mathrm{TSS}=9$ & Indigo $2 \%$ \\
\hline & $\mathrm{MSS}=4$ & Indigo $2 \%$ & & $\mathrm{MSS}=6$ & $\mathrm{TSS}=10$ \\
\hline & & $\mathrm{TSS}=8$ & & & $\mathrm{MSS}=5$ \\
\hline & & $\mathrm{MSS}=5$ & Trophy & Gold $73 \%$ & Gold $72 \%$ \\
\hline \multirow[t]{8}{*}{ Riches } & Green $41 \%$ & Green $37 \%$ & & Yellow 14 \% & Yellow $20 \%$ \\
\hline & Gold $31 \%$ & Gold $47 \%$ & & Silver $6 \%$ & Silver $2 \%$ \\
\hline & Yellow $16 \%$ & Yellow $6 \%$ & & Red $2 \%$ & Green $4 \%$ \\
\hline & Red $4 \%$ & Red $10 \%$ & & Black $2 \%$ & Gray $2 \%$ \\
\hline & Blue $6 \%$ & $\mathrm{TSS}=4$ & & Bronze $2 \%$ & $\mathrm{TSS}=5$ \\
\hline & White $2 \%$ & $\mathrm{MSS}=4$ & & $\mathrm{TSS}=6$ & $\mathrm{MSS}=3$ \\
\hline & $\mathrm{TSS}=6$ & & & $\mathrm{MSS}=3$ & \\
\hline & $\mathrm{MSS}=5$ & & Vacation & Blue $52 \%$ & Blue $44 \%$ \\
\hline \multirow[t]{7}{*}{ Romantic } & Red $90 \%$ & Red $84 \%$ & & Yellow 29 \% & Yellow $26 \%$ \\
\hline & Pink $6 \%$ & Pink $10 \%$ & & Red $2 \%$ & Red $6 \%$ \\
\hline & Blue $2 \%$ & Wine Red $2 \%$ & & Orange $6 \%$ & Orange $2 \%$ \\
\hline & White $2 \%$ & Black $2 \%$ & & White $4 \%$ & White $2 \%$ \\
\hline & $\mathrm{TSS}=4$ & Purple $2 \%$ & & Green $2 \%$ & Green $4 \%$ \\
\hline & $\mathrm{MSS}=2$ & $\mathrm{TSS}=5$ & & Purple $2 \%$ & Purple $2 \%$ \\
\hline & & $\mathrm{MSS}=2$ & & Beige $2 \%$ & Pink $6 \%$ \\
\hline \multirow[t]{11}{*}{ Thrill } & Red $48 \%$ & Red $54 \%$ & & Black $2 \%$ & Tan $4 \%$ \\
\hline & Blue $12 \%$ & Blue $16 \%$ & & $\mathrm{TSS}=9$ & Beige $2 \%$ \\
\hline & Yellow $14 \%$ & Yellow 6 \% & & $\mathrm{MSS}=4$ & Gold $2 \%$ \\
\hline & Green $6 \%$ & Green $10 \%$ & & & $\mathrm{TSS}=11$ \\
\hline & Orange $6 \%$ & Orange $8 \%$ & & & $\mathrm{MSS}=6$ \\
\hline & Black $8 \%$ & Black $4 \%$ & Victory & Red $33 \%$ & $\operatorname{Red} 28 \%$ \\
\hline & Forrest Green $2 \%$ & Silver $2 \%$ & & Gold $23 \%$ & Gold $20 \%$ \\
\hline & Aqua $2 \%$ & $\mathrm{TSS}=7$ & & Blue $16 \%$ & Blue $24 \%$ \\
\hline & Purple $2 \%$ & $\mathrm{MSS}=6$ & & White $14 \%$ & White $6 \%$ \\
\hline & $\mathrm{TSS}=9$ & & & Yellow $4 \%$ & Yellow $10 \%$ \\
\hline & $\mathrm{MSS}=6$ & & & Green $2 \%$ & Green $6 \%$ \\
\hline \multirow[t]{10}{*}{ Treasure } & Gold $74 \%$ & Gold $71 \%$ & & Silver $4 \%$ & Black $2 \%$ \\
\hline & Brown $12 \%$ & Brown $6 \%$ & & Pink $2 \%$ & Orange $2 \%$ \\
\hline & Yellow $6 \%$ & Yellow $10 \%$ & & Brown $2 \%$ & Violet $2 \%$ \\
\hline & Green $2 \%$ & Green $2 \%$ & & $\mathrm{TSS}=9$ & $\mathrm{TSS}=9$ \\
\hline & White $2 \%$ & Pink $4 \%$ & & $\mathrm{MSS}=6$ & $\mathrm{MSS}=6$ \\
\hline & Pearl White $2 \%$ & Blue $2 \%$ & Waterfall & Blue $76 \%$ & Blue $82 \%$ \\
\hline & $\operatorname{Red} 2 \%$ & Purple $2 \%$ & & White $14 \%$ & White $10 \%$ \\
\hline & $\mathrm{TSS}=7$ & Maroon $2 \%$ & & Gray $4 \%$ & Gray $2 \%$ \\
\hline & $\mathrm{MSS}=3$ & $\mathrm{TSS}=8$ & & Aqua $2 \%$ & Clear $4 \%$ \\
\hline & & $\mathrm{MSS}=4$ & & Forrest Green $2 \%$ & Green $2 \%$ \\
\hline \multirow[t]{4}{*}{ Triumph } & Yellow $33 \%$ & Yellow $26 \%$ & & Rainbow $2 \%$ & $\mathrm{TSS}=5$ \\
\hline & Gold $23 \%$ & Gold $30 \%$ & & $\mathrm{TSS}=6$ & $\mathrm{MSS}=3$ \\
\hline & Red $18 \%$ & Red $14 \%$ & & $\mathrm{MSS}=3$ & \\
\hline & Blue $8 \%$ & Blue $16 \%$ & Wedding & White $94 \%$ & White $96 \%$ \\
\hline
\end{tabular}

Table 7 (continued) 
Table 7 (continued)

\begin{tabular}{|c|c|c|}
\hline Word & Sample A & Sample B \\
\hline \multirow{16}{*}{ Wish } & Red $4 \%$ & Purple $2 \%$ \\
\hline & Gold $2 \%$ & Black $2 \%$ \\
\hline & $\mathrm{TSS}=3$ & $\mathrm{TSS}=3$ \\
\hline & $\mathrm{MSS}=2$ & $\mathrm{MSS}=1$ \\
\hline & White $35 \%$ & White $31 \%$ \\
\hline & Blue $21 \%$ & Blue $25 \%$ \\
\hline & Yellow $17 \%$ & Yellow $12 \%$ \\
\hline & Green $6 \%$ & Green $9 \%$ \\
\hline & Gold $4 \%$ & Gold $9 \%$ \\
\hline & Red $4 \%$ & Red $8 \%$ \\
\hline & Pink $8 \%$ & Pink $2 \%$ \\
\hline & Cream $2 \%$ & Orange $2 \%$ \\
\hline & Silver $2 \%$ & $\mathrm{TSS}=8$ \\
\hline & Purple $2 \%$ & $\mathrm{MSS}=6$ \\
\hline & $\mathrm{TSS}=10$ & \\
\hline & $\mathrm{MSS}=7$ & \\
\hline
\end{tabular}

\section{References}

Altarriba, J., \& Basnight-Brown, D. M. (2011). The representation of emotion vs. emotion-laden words in English and in Spanish in the affective Simon task. International Journal of Bilingualism, 15, $310-328$

Baddeley, A. D. (1972). Selective attention and performance in dangerous environments. British Journal of Psychology, 63, 537-546.

Bartels, A., \& Zeki, S. (2000). The architecture of the color centre in the human visual brain: New results and a review. European Journal of Neuroscience, 12, 172-193.

Bellizzi, J. A., \& Hite, R. E. (1992). Environmental color, consumer feelings, and purchase likelihood. Psychology and Marketing, 9, 347-363.

Bramão, I., Faísca, L., Forkstam, C., Inácio, F., Araújo, S., Petersson, K. M., \& Reis, A. (2012). The interaction between surface color and color knowledge: Behavioral and electrophysiological evidence. Brain and Cognition, 78, 28-37.

Collins, A. M., \& Loftus, E. F. (1975). A spreading-activation theory of semantic processing. Psychological Review, 82, 407-428. doi:10. 1037/0033-295X.82.6.407

Elliot, A. J., \& Maier, M. A. (2007). Color and psychological functioning. Current Directions in Psychological Science, 16, 250-254.

Elliot, A. J., Maier, M. A., Moller, A. C., Friedman, R., \& Meinhardt, J. (2007). Color and psychological functioning: The effect of red on performance attainment. Journal of Experimental Psychology: General, 136, 154-168. doi:10.1037/0096-3445.136.1.154

Gegenfurtner, K. R., \& Kiper, D. C. (2003). Color vision. Annual Review of Neuroscience, 26, 181-206. doi:10.1146/annurev.neuro.26. 041002.131116

Gohar, K. N. (2008). Diagnostic colours of emotions (Doctoral dissertation). Sydney: University of Sydney.
Hemphill, M. (1996). A note $n$ adults' color-emotion associations. Journal of Genetic Psychology, 157, 275-280.

Hupka, R. B., Zaleski, Z., Otto, J., Reidl, L., \& Tarabrina, N. V. (1997). The colors of anger, envy, fear, and jealousy. Journal of CrossCultural Psychology, 28, 156-171. doi:10.1177/ 0022022197282002

Indow, T. (1988). Multidimensional studies of Munsell color solid. Psychological Review, 95, 456-470. doi:10.1037/0033-295X.95.4. 456

Keil, A., \& Ihssen, N. (2004). Identification facilitation for emotionally arousing verbs during the attentional blink. Emotion, 4, 23-35.

Knickerbocker, H., \& Altarriba, J. (2013). Differential repetition blindness with emotion and emotion-laden wordtypes. Visual Cognition, 21, 599-627.

Kuhbandner, C., \& Pekrun, R. (2013). Joint effects of emotion and color on memory. Emotion, 13, 375-379.

McKenna, F. P. (1986). Effects of unattended emotional stimuli on colornaming performance. Current Psychological Research and Reviews, 5, 3-9.

McKenna, F. P., \& Sharma, D. (1995). Intrusive cognitions: An investigation of the emotional Stroop task. Journal of Experimental Psychology: Learning, Memory, and Cognition, 21, 1595-1607. doi:10.1037/0278-7393.21.6.1595

Meier, B. P., Robinson, M. D., \& Clore, G. L. (2004). Why good guys wear white: Automatic inferences about stimulus valence based on brightness. Psychological Science, 15, 82-87. doi:10.1111/j.09637214.2004.01502002.x

Moller, A. C., Elliot, A. J., \& Maier, M. A. (2009). Basic hue-meaning associations. Emotion, 9, 898-902.

Nelson, D. L., McEvoy, C. L., \& Schreiber, T. A. (1998). The University of South Florida word association, rhyme, and word fragment norms [Database]. Retrieved from http://w3.usf.edu/FreeAssociation/

Nelson, D. L., \& Schreiber, T. A. (1992). Word concreteness and word structure as independent determinants of recall. Journal of Memory and Language, 31, 237-260. doi:10.1016/0749-596X(92)90013-N

Ostergaard, A. L., \& Davidoff, J. B. (1985). Some effects of color on naming and recognition of objects. Journal of Experimental Psychology: Learning, Memory, and Cognition, 11, 579-587. doi: 10.1037/0278-7393.11.3.579

Sutton, T. M., \& Altarriba, J. (2008). Emotion words in the mental lexicon: A new look at the emotional Stroop effect. Mental Lexicon, 3, 29-46.

Tanaka, J. W., \& Presnell, L. M. (1999). Color diagnosticity in object recognition. Perception \& Psychophysics, 61, 1140-1153.

Travis, D. (1991). Effective color displays: Theory and practice. London: Academic Press.

Van Overschelde, J. P., Rawson, K. A., \& Dunlosky, J. (2004). Category norms: An updated and expanded version of the Battig and Montague (1969) norms. Journal of Memory and Language, 50, 289-335. doi:10.1016/j.jml.2003.10.003

Vuilleumier, P. (2005). How brains beware: Neural mechanisms of emotional attention. Trends in Cognitive Sciences, 9, 585-594. doi:10. 1016/j.tics.2005.10.011

Wurm, L. H., Legge, G. E., Isenberg, L. M., \& Luebker, A. (1993). Color improves object recognition in normal and low vision. Journal of Experimental Psychology: Human Perception and Performance, 19, 899-911. doi:10.1037/0096-1523.19.4.899

Young, S. G., Elliot, A. J., Feltman, R., \& Ambady, N. (2013). Red enhances the processing of facial expressions of anger. Emotion, $13,380-384$. 